\title{
Estabilidade estrutural dos campos vetoriais seccionalmente lineares no plano
}

\author{
Bruno de Paula Jacóia
}

\author{
DiSSERTAÇÃO APRESENTADA \\ $\mathrm{AO}$ \\ Instituto DE MATEMÁTICA E EstATÍSTICA \\ DA \\ UNIVERSIDADE DE SÃO PAULO \\ PARA \\ OBTENÇÃO DO TÍTULO \\ DE \\ Mestre EM CiênCIAS
}

\author{
Programa: Matemática Aplicada \\ Orientador: Prof. Dr. Jorge Manuel Sotomayor Tello
}

Durante o desenvolvimento deste trabalho o autor recebeu auxílio financeiro do CNPq São Paulo, julho de 2013 



\section{Estabilidade estrutural dos campos vetoriais seccionalmente lineares no plano}

Esta versão da dissertação contém as correções e alterações sugeridas pela Comissão Julgadora durante a defesa da versão original do trabalho, realizada em 15 de agosto de 2013. Uma cópia da versão original está disponível no

Instituto de Matemática e Estatística da Universidade de São Paulo.

Comissão Julgadora:

- Prof. Dr. Jorge Manuel Sotomayor Tello - IME-USP

- Prof. Dr. Salvador Addas Zanata - IME-USP

- Prof. Dr. Ronaldo Alves Garcia - UFG 



\section{Agradecimentos}

Primeiramente gostaria de agradecer ao meu orientador, Prof. Dr. Jorge Manuel Sotomayor Tello, pela sua dedicação, atenção, paciência e pelos muitos conselhos e ensinamentos transmitidos.

Agradeço ao Prof. Dr. Ronaldo Alves Garcia, pelas valiosas observações e sugestões que ajudaram a aprimorar este trabalho.

Agradeço a toda minha família, especialmente aos meus pais, Zé e Cida, e aos meus avós, Antônio e Antonia, que sempre estão presentes me incentivando e torcendo por mim.

Agradeço a Priscila, que me acompanhou durante todo esse caminho, me apoiando e incentivando, nos bons e maus momentos, sendo uma fonte inesgotável de carinho.

Agradeço também ao Ramón, pelos inúmeros momentos de alegria proporcionados.

E por último, mas não menos importante, gostaria também de agradecer a todos os amigos que encontrei no instituto: Pedro, Lucas, Henrique, Ivã, Arthur, Gabriel, Luís, Max, Gabriel, Lucas, Ricardo, Fernando, Yuri, Felipe, Godoy, Fábio, André e Belmiro. Sem vocês todos esses anos no IME não teriam sido tão alegres e proveitosos. 


\section{Resumo}

\section{JACÓIA, B. P. Estabilidade Estrutural dos Campos Vetoriais Seccionalmente}

Lineares no Plano. 2013. Dissertação (Mestrado) - Instituto de Matemática e Estatística, Universidade de São Paulo, São Paulo, 2013.

Estudamos uma classe de campos de vetores seccionalmente lineares no plano denotada por $\mathfrak{X}_{\Gamma, \Delta}$. Tais campos aparecem frequentemente em modelos matemáticos aplicados à engenharia. Baseados no trabalho de J. Sotomayor e R. Garcia [SG03], impondo condições sobre as singularidades, órbitas periódicas e separatrizes, definimos um conjunto $\Sigma_{\Gamma, \Delta}$ de campos de vetores que são estruturalmente estáveis em $\mathfrak{X}_{\Gamma, \Delta}$. Provamos que esse conjunto é aberto, denso e tem medida de Lebesgue total em $\mathfrak{X}_{\Gamma, \Delta}$, o qual é um espaço vetorial de dimensão finita.

Palavras-chave: Estabilidade Estrutural, Campos de vetores lineares por partes, Compactificação de Poincaré. 


\section{Abstract}

JACÓIA, B. P. Structural Stability of Piecewise-linear Vector Fields in the Plane. 2013. Dissertação (Mestrado) - Instituto de Matemática e Estatística, Universidade de São Paulo, São Paulo, 2013.

We study a class of Piecewise-linear vector fields in the plane denoted by $\mathfrak{X}_{\Gamma, \Delta}$. These vector fields appear often in mathematical models applied to Engineering. Based on Jorge Sotomayor and Ronaldo Garcia paper [SG03], we impose conditions on singularities, periodic orbits and separatrices, to define a set $\Sigma_{\Gamma, \Delta}$ of vector fields structurally stable in $\mathfrak{X}_{\Gamma, \Delta}$. We give a proof that this set is open, dense and has full Lebesgue measure in $\mathfrak{X}_{\Gamma, \Delta}$, that is a finite dimensional vector space.

Palavras-chave: Structural Stability, Piecewise-linear Vector Fields, Poincaré Compactification. 


\section{Sumário}

1 Introdução 1

2 Preliminares 5

2.1 Campos seccionalmente lineares no plano . . . . . . . . . . . . 5

2.2 Resultados clássicos . . . . . . . . . . . . . . . . . . . . . . . 8

2.3 Aplicação de transição . . . . . . . . . . . . . . . . . . . . . . . . . . . . . . . .

2.3.1 Derivada da aplicação de transição . . . . . . . . . . . . . . . . . . 14

2.3.2 Derivada da aplicação de retorno para órbitas periódicas . . . . . . 16

2.4 Compactificação de Poincaré . . . . . . . . . . . . . . . . . . . . . . 18

2.5 Comportamento dos campos no infinito via compactificação . . . . . . . . 23

2.5.1 Derivada da aplicação de retorno para a órbita no infinito . . . . . 31

2.6 Tangências com a grade . . . . . . . . . . . . . . . . . . 35

$\begin{array}{lll}3 & \text { Estabilidade Estrutural } & 37\end{array}$

3.1 Definição de Estabilidade Estrutural . . . . . . . . . . . . . . . . . 37

3.2 Propriedades de genericidade dos sistemas seccionalmente lineares . . . . . 38

3.3 Teorema de Estabilidade e Genericidade . . . . . . . . . . . . . . . . . . 39

3.4 Lemas . . . . . . . . . . . . . . . . . . . . . . . . . 41

4 Abertura, Densidade e Estabilidade de $\Sigma_{\Gamma, \Delta} \quad 57$

4.1 Abertura . . . . . . . . . . . . . . . . . . . 57

4.2 Regiões canônicas . . . . . . . . . . . . . . . . . . . . 57

4.2.1 Construção do homeomorfismo . . . . . . . . . . . . . . . 63

4.3 Densidade . . . . . . . . . . . . . . . . . . . . . . . . 69

$\begin{array}{llr}5 & \text { Conclusões } & 87\end{array}$ 


\section{Capítulo 1}

\section{Introdução}

A forma como as equações diferenciais ordinárias eram estudadas mudou drasticamente no final do século XIX depois que Henri Poincaré publicou seu seminal trabalho "Mémoire sur les courbes définies par une équation différentielle" em 1881.

Este trabalho introduziu novas técnicas e uma inovadora linha de pensamento para o estudo das equações diferenciais ordinárias, e teve uma enorme influência para este ramo da matemática criando os alicerces da hoje conhecida Teoria Qualitativa das Equações Diferenciais e Sistemas Dinâmicos.

Tornou-se então importante a compreensão, geométrica e analítica, dos retratos de fase dos sistemas dinâmicos definidos por equações diferenciais, bem como o comportamento destes quando as equações que definem a dinâmica sofrem pequenas perturbações.

Este último aspecto se deu numa fase posterior do amadurecimento e assimilação das ideias de Poincaré.

Assim, estudos pioneiros iniciados por Alexander Andronov e Lev Pontryagin em 1937 deram origem ao conceito hoje conhecido como estabilidade estrutural dos sistemas dinâmicos.

Eles estavam interessados em entender como pequenas perturbações nas equações que definem o sistema afetam qualitativamente o comportamento de suas soluções e qual tipo de sistema é "robusto" o suficiente para que seus aspectos qualitativos permaneçam inalterados após tais perturbações.

Inspirados por essa forma de analisar os campos, dizemos que um sistema é estruturalmente estável caso as propriedades topológicas do sistema dinâmico se mantenham as mesmas após uma pequena perturbação da função que os definem.

No início da década de 1960, os matemáticos brasileiros Maurício Peixoto e Marilia Peixoto, estendendo os resultados dos trabalhos de Andronov e Pontryagin, demonstraram em [PP59] um teorema de necessidade e suficiência para a estabilidade estrutural de sistemas dinâmicos em regiões compactas com bordo suave do plano.

No trabalho de Peixoto e Peixoto a compreensão do conjunto dos sistemas estrutural- 
mente estáveis é obtida através da caracterização das condições que elementos essenciais do sistema (singularidades, órbitas periódicas e separatrizes) devem satisfazer. Além disso é provado em [Pei62] que os sistemas estruturalmente estáveis formam um conjunto aberto e denso no espaço dos sistemas considerados.

Posteriormente Jorge Sotomayor estendeu o resultado para o caso de sistemas polinomiais definidos no plano $\mathbb{R}^{2}$.

A caracterização dos sistemas estruturalmente estáveis e sua densidade é uma questão de grande relevância para várias aplicações. Por exemplo, em sistemas oriundos da física ou da biologia, podemos analisar o comportamento do retrato de fase de tais sistemas fazendo aproximações e medições, e nesse caso podemos considerar que o sistema sofre pequenas perturbações devidas a pequenos erros das medições ou limitações de precisão dos equipamentos utilizados. Portanto contar a estabilidade estrutural do sistema nos garante que tais perturbações não afetam qualitativamente o comportamento assintótico das soluções.

Esta dissertação se baseia no artigo [SG03] de Jorge Sotomayor e Ronaldo Garcia. Serão considerados neste trabalho os campos vetoriais contínuos seccionalmente lineares no plano. Em [CL91], L. Chua e R. Lum discutem o interesse computacional e a aplicabilidade de tais sistemas.

Tais campos aparecem persistentemente em problemas envolvendo teoria do controle e em modelagens matemáticas de problemas de engenharia que possuem comportamento não suave.

Logo, é de grande interesse aplicado, caracterizarmos os campos de vetores que são estruturalmente estáveis dentro da classe dos campos de vetores seccionalmente lineares no plano.

A dissertação está organizada da seguinte maneira.

No capítulo 2, introduzimos o tipo de campos de vetores que estudaremos e sua representação matemática. Definimos também qual será o conjunto onde os campos deixam de ser diferenciáveis. Observamos que tais campos formam um espaço vetorial de dimensão finita, representado por $\mathfrak{X}_{\Gamma, \Delta}$, e portanto podemos associá-los naturalmente com o espaço euclidiano de dimensão apropriada.

É introduzido também uma importante ferramenta, chamada de compactificação de Poincaré, apropriada para lidar com a não compacidade do plano de fase dos sistemas em estudo.

Também enunciaremos e provaremos alguns resultados técnicos referentes aos campos em questão. Estabeleceremos a relação entre alguns resultados sobre sistemas dinâmicos suaves e seus correspondentes no caso de campos seccionalmente lineares no plano.

Faremos a análise matemática e daremos a descrição geométrica de como alguns tipos de perturbação afetam os campos estudados, também introduziremos ferramentas que facilitam a análise do comportamento de singularidades e órbitas periódicas dos sistemas em 
questão, principalmente a análise dos resultados referentes ao comportamento no equador da esfera $\mathbb{S}^{2}$ depois que os campos são compactificados.

No capítulo 3 é definido o que é estabilidade estrutural dentro da classe dos campos de vetores seccionalmente lineares no plano. Inspirados pelos trabalhos de Maurício Peixoto, Marília Peixoto, Jorge Sotomayor e Ronaldo Garcia, descrevemos quais são as propriedades que um campo de vetores deve satisfazer para ser estruturalmente estável. Essas propriedades são relativas a elementos dinâmicos essenciais do sistema, tais como as singularidades, órbitas periódicas e separatrizes.

Neste capítulo é enunciado o principal teorema deste trabalho, que estabelece as propriedades topológicas e a medida de Lebesgue do conjunto $\Sigma_{\Gamma, \Delta}$ de campos estruturalmente estáveis, tal como definido em 3.1.

Devido ao fato do espaço em questão ser de dimensão finita, além de provar que o conjunto descrito é aberto e denso,conseguiremos mostrar mediante uma análise adicional, que o complementar deste conjunto tem medida de Lebesgue nula no espaço dos campos de vetores seccionalmente lineares no plano.

No capítulo 4 é provado o principal teorema deste trabalho. Adaptamos a técnica das regiões canônicas encontrada no trabalho de Peixoto e Peixoto para construir os homeomorfismos que estabelecem a equivalência topológica entre campos próximos em $\Sigma_{\Gamma, \Delta}$. Por fim, através da análise do comportamento de uma família de campos dependendo de cinco parâmetros, provamos que o conjunto $\Sigma_{\Gamma, \Delta}$ possui medida de Lebesgue total, e em particular, é denso em $\mathfrak{X}_{\Gamma, \Delta}$.

No capítulo 5 , fazemos uma síntese dos resultados demonstrados e apontamos possíveis linhas de estudo e trabalho a seguir. 


\section{Capítulo 2}

\section{Preliminares}

Neste capítulo definimos a classe dos campos de vetores seccionalmente lineares no plano. Também apresentamos alguns resultados e construções que nos fornecerão ferramentas úteis para analisar o comportamento do retrato de fase de tais sistemas, focando nos em seus elementos dinâmicos essenciais, tais como os pontos singulares, órbitas periódicas e separatrizes.

Construímos a aplicação de transição entre duas seções transversais distintas do campo, e demonstramos a fórmula para a sua derivada. Apresentamos o método da compactificação de Poincaré, ferramenta adequada para lidarmos com a não compacidade do plano.

Terminamos o capítulo fazendo o estudo do comportamento da compactificação dos campos seccionalmente lineares no plano e a mudança de seus retratos de fase através de alguns tipos de perturbações.

\subsection{Campos seccionalmente lineares no plano}

No plano $\mathbb{R}^{2}$ com as coordenadas $(x, y)$ considere o campo de vetores

$$
X(x, y)=(P(x, y), Q(x, y))
$$

onde $P$ e $Q$ são dados por

$$
\begin{aligned}
& P(x, y)=a_{1}+b_{11} x+b_{12} y+\sum_{i=1}^{n} c_{1}^{i}\left|x-\gamma_{i}\right|+\sum_{j=1}^{m} d_{1}^{j}\left|y-\delta_{j}\right| \\
& Q(x, y)=a_{2}+b_{21} x+b_{22} y+\sum_{i=1}^{n} c_{2}^{i}\left|x-\gamma_{i}\right|+\sum_{j=1}^{m} d_{2}^{j}\left|y-\delta_{j}\right| .
\end{aligned}
$$


Denotaremos por $\Gamma=\left\{\gamma_{1}<\gamma_{2}<\ldots<\gamma_{n}\right\}$ e $\Delta=\left\{\delta_{1}<\delta_{2}<\ldots<\delta_{m}\right\}$ as sequências ordenadas de números reais que definem as retas $x=\gamma_{i}$ e $y=\delta_{j}$ fora das quais o campo de vetores $X$ é linear e portanto suave.

No plano essas retas definem uma grade que será denotada por $[\Gamma, \Delta]$. Os pontos da forma $\left(\gamma_{i}, \delta_{j}\right), i \in\{1, \ldots, n\} ; j \in\{1, \ldots, m\}$; serão chamados de pontos de esquina da grade. O conjunto formado por todos os pontos de esquina da grade será denotado por $\Gamma \times \Delta$.

Fixados os conjuntos $\Gamma$ e $\Delta$, denotaremos por $\mathfrak{X}_{\Gamma, \Delta}$ o espaço de todos os campos de vetores definidos como em 2.1, fixada a grade $[\Gamma, \Delta]$.

$\mathfrak{X}_{\Gamma, \Delta}$ é um espaço vetorial sobre $\mathbb{R}$ de dimensão $N(\Gamma, \Delta)=6+2(n+m)$. De fato podemos naturalmente associar a cada campo de vetores em $\mathfrak{X}_{\Gamma, \Delta}$ um elemento de

$$
\mathbb{R}^{2} \times \mathbb{R}^{4} \times \mathbb{R}^{n} \times \mathbb{R}^{n} \times \mathbb{R}^{m} \times \mathbb{R}^{m} \simeq \mathbb{R}^{6+2(n+m)}=\mathbb{R}^{N(\Gamma, \Delta)}
$$

Fazemos isso usando seus parâmetros

$$
\begin{gathered}
\left(a_{1}, a_{2}\right) \in \mathbb{R}^{2}, \\
\left(b_{11}, b_{12}, b_{21}, b_{22}\right) \in \mathbb{R}^{4} \\
\left(c_{1}^{1}, c_{1}^{2}, \ldots, c_{1}^{n}\right) e\left(c_{2}^{1}, c_{2}^{2}, \ldots, c_{2}^{n}\right) \in \mathbb{R}^{n} \\
\left(d_{1}^{1}, d_{1}^{2}, \ldots, d_{1}^{m}\right) e\left(d_{2}^{1}, d_{2}^{2}, \ldots, d_{2}^{m}\right) \in \mathbb{R}^{m} .
\end{gathered}
$$

Devido a essa natureza do espaço em questão, podemos ir mais adiante e considerá-lo como um espaço métrico com a norma euclidiana herdada de $\mathbb{R}^{N(\Gamma, \Delta)}$. Denotaremos por $B_{\epsilon}(X)$ a bola aberta em $\mathfrak{X}_{\Gamma, \Delta}$ centrada em $X$ e de raio $\epsilon>0$.

Observe que os campos de vetores em $\mathfrak{X}_{\Gamma, \Delta}$ são contínuos e seccionalmente lineares. A grade $[\Gamma, \Delta]$ particiona o plano $\mathbb{R}^{2}$ em $(n+1)(m+1)$ células $\Omega_{i j}=\Gamma_{i} \times \Delta_{j}$ e restrito a cada uma dessas células o campo é linear não homogêneo, ou seja

$$
\left.X(x, y)\right|_{\Omega_{i j}}=A_{i j}(x y)^{T}+b_{i j}
$$

$\operatorname{com} A_{i j} \in \mathbb{M}_{2}(\mathbb{R})$ e $b_{i j} \in \mathbb{R}^{2}$.

Aqui, $\Gamma_{i}=\left[\gamma_{i}, \gamma_{i+1}\right], i=1,2, \ldots, n-1, \Gamma_{0}=\left[-\infty, \gamma_{1}\right]$ e $\Gamma_{n}=\left[\gamma_{n}, \infty\right]$. Analogamente, $\Delta_{j}=\left[\delta_{j}, \delta_{j+1}\right], j=1,2, \ldots, m-1, \Delta_{0}=\left[-\infty, \delta_{1}\right]$ e $\Delta_{n}=\left[\delta_{m}, \infty\right]$.

Por exemplo, se $(x, y) \in \Omega_{k_{x}, k_{y}}$, então temos $\gamma_{1}<\ldots<\gamma_{k_{x}} \leqslant x \leqslant \gamma_{k_{x}+1}<\ldots<\gamma_{n}$ e $\delta_{1}<\ldots<\delta_{k_{y}} \leqslant y \leqslant \delta_{k_{y}+1}<\ldots<\delta_{m}$.

Substituindo na expressão do campo $X$ dado em 2.1 temos 


$$
\begin{aligned}
P(x, y)= & {\left[a_{1}-\sum_{i=1}^{k_{x}} c_{1}^{i} \gamma_{i}+\sum_{i=k_{x}+1}^{n} c_{1}^{i} \gamma_{i}-\sum_{j=1}^{k_{y}} d_{1}^{j} \delta_{j}+\sum_{j=k_{y}+1}^{m} d_{1}^{j} \delta_{j}\right]+} \\
& +\left[b_{11}+\sum_{i=1}^{k_{x}} c_{1}^{i}-\sum_{i=k_{x}+1}^{n} c_{1}^{i}\right] x+\left[b_{12}+\sum_{j=1}^{k_{y}} d_{1}^{j}-\sum_{j=k_{y}+1}^{m} d_{1}^{j}\right] y \\
Q(x, y)= & {\left[a_{2}-\sum_{i=1}^{k_{x}} c_{2}^{i} \gamma_{i}+\sum_{i=k_{x}+1}^{n} c_{2}^{i} \gamma_{i}-\sum_{j=1}^{k_{y}} d_{2}^{j} \delta_{j}+\sum_{j=k_{y}+1}^{m} d_{2}^{j} \delta_{j}\right]+} \\
& +\left[b_{21}+\sum_{i=1}^{k_{x}} c_{2}^{i}-\sum_{i=k_{x}+1}^{n} c_{2}^{i}\right] x+\left[b_{22}+\sum_{j=1}^{k_{y}} d_{2}^{j}-\sum_{j=k_{y}+1}^{m} d_{2}^{j}\right] y .
\end{aligned}
$$

Então o campo $\left.X(x, y)\right|_{\Omega_{i j}}=\left(\begin{array}{cc}A_{11} & A_{12} \\ A_{21} & A_{22}\end{array}\right)\left(\begin{array}{l}x \\ y\end{array}\right)+\left(\begin{array}{c}B_{1} \\ B_{2}\end{array}\right)$, com os seguintes coeficientes

$$
\begin{gathered}
A_{11}=b_{11}+\sum_{i=1}^{k_{x}} c_{1}^{i}-\sum_{i=k_{x}+1}^{n} c_{1}^{i}, \\
A_{12}=b_{12}+\sum_{j=1}^{k_{y}} d_{1}^{j}-\sum_{j=k_{y}+1}^{m} d_{1}^{j}, \\
A_{21}=b_{21}+\sum_{i=1}^{k_{x}} c_{2}^{i}-\sum_{i=k_{x}+1}^{n} c_{2}^{i}, \\
A_{22}=b_{22}+\sum_{j=1}^{k_{y}} d_{2}^{j}-\sum_{j=k_{y}+1}^{m} d_{2}^{j}, \\
B_{1}=a_{1}-\sum_{i=1}^{k_{x}} c_{1}^{i} \gamma_{i}+\sum_{i=k_{x}+1}^{n} c_{1}^{i} \gamma_{i}-\sum_{j=1}^{k_{y}} d_{1}^{j} \delta_{j}+\sum_{j=k_{y}+1}^{m} d_{1}^{j} \delta_{j}, \\
B_{2}=a_{2}-\sum_{i=1}^{k_{x}} c_{2}^{i} \gamma_{i}+\sum_{i=k_{x}+1}^{n} c_{2}^{i} \gamma_{i}-\sum_{j=1}^{k_{y}} d_{2}^{j} \delta_{j}+\sum_{j=k_{y}+1}^{m} d_{2}^{j} \delta_{j} .
\end{gathered}
$$

As células $\Omega_{i j}$ que são limitadas serão chamadas de finitas, e as células que não são limitadas serão chamadas de infinitas.

Observe que todo $X \in \mathfrak{X}_{\Gamma, \Delta}$ é um campo de vetores lipschitziano em $\mathbb{R}^{2}$. De fato, em cada célula $\Omega_{i j}$ o campo é linear não homogêneo, logo podemos tomar como constante de Lipschitz para $X$ o valor

$$
L=\max \left\{\left\|A_{i j}\right\|, 1 \leqslant i \leqslant n, 1 \leqslant j \leqslant m\right\}
$$

onde estamos considerando a norma das aplicações lineares de $\mathbb{R}^{2}$. 
Logo para todo $X \in \mathfrak{X}_{\Gamma, \Delta}$ existe um único fluxo do sistema, bem definido e completo. Isto é, para todo $(x, y) \in \mathbb{R}^{2}$ a curva integral do campo $X$ passando por $(x, y)$ está definida para todo $t \in \mathbb{R}$.

Nosso interesse no estudo desses campos é definir e analisar a estabilidade estrutural em $\mathfrak{X}_{\Gamma, \Delta}$, bem como encontrar quais são as condições que um campo de vetores $X$ precisa satisfazer para que ele seja estruturalmente estável nesse conjunto.

Para tanto é de suma importância entendermos o retrato de fase de $X$, em particular como é o comportamento em torno de suas singularidades (ou equilíbrios), suas órbitas periódicas, suas conexões de separatrizes e o comportamento das órbitas com pontos limites no infinito.

\subsection{Resultados clássicos}

Nesta seção apresentaremos alguns resultados clássicos da teoria qualitativa de equações diferenciais que servirão de ferramentas para analisarmos os campos de vetores seccionalmente lineares no plano.

Teorema 2.1 (Continuidade com relação aos parâmetros e condições iniciais) Seja $F$ uma função contínua num conjunto aberto $U \subseteq \mathbb{R} \times \mathbb{R}^{n} \times \mathbb{R}^{m}$. Para cada $\left(t_{0}, p_{0}, \lambda\right) \in$ $U$ suponha que o problema de valores iniciais

$$
p^{\prime}=F(t, p, \lambda), \quad p\left(t_{0}\right)=p_{0}
$$

tenha uma única solução $\varphi=\varphi\left(t ; t_{0}, p_{0}, \lambda\right)$, definida no seu intervalo máximo

$$
\left(\omega_{-}\left(t_{0}, p_{0}, \lambda\right), \omega_{+}\left(t_{0}, p_{0}, \lambda\right)\right)
$$

Então

$$
\mathscr{D}=\left\{\left(t, t_{0}, p_{0}, \lambda\right) \mid\left(t_{0}, p_{0}, \lambda\right) \in U \text { e } t \in\left(\omega_{-}\left(t_{0}, p_{0}, \lambda\right), \omega_{+}\left(t_{0}, p_{0}, \lambda\right)\right)\right\}
$$

é aberto em $\mathbb{R} \times U$ e $\varphi$ é contínua em $\mathscr{D}$.

Demonstração: A demonstração deste teorema pode ser vista em [Sot79].

Observação 2.1 O teorema 2.1 nos permite tratar os campos em $\mathfrak{X}_{\Gamma, \Delta}$ como campos de 
vetores dependendo de $N(\Gamma, \Delta)$ parâmetros.

Quando quisermos destacar que estamos usando essa propriedade escreveremos o fluxo de um campo $X \in \mathfrak{X}_{\Gamma, \Delta}$ como sendo $\varphi(t, p, X)$. Para deixar claro que estamos usando o campo de vetores em $\mathfrak{X}_{\Gamma, \Delta}$ como parâmetro iremos dizer que estamos usando a propriedade da continuidade do fluxo com relação aos campos ao invés de dizer com relação aos parâmetros.

Teorema 2.2 (Poincaré-Bendixson) Sejam $X$ um campo de vetores localmente lipschtziano definido em um aberto $U \subseteq \mathbb{R}^{2}$ e $\gamma^{+}(p)=\{\varphi(t, p) \mid t \geqslant 0\}$ (respectivamente $\left.\gamma^{-}(p)=\{\varphi(t, p) \mid t \leqslant 0\}\right)$ a semi-órbita positiva (respectivamente a semi-órbita negativa) do campo X pelo ponto $p$. Suponha que $\gamma^{+}(p)$ (respectivamente $\gamma^{-}(p)$ ) está contida num subconjunto compacto de $U$ e $\omega(p)$ (respectivamente $\alpha(p)$ ) tem um número finito de pontos singulares. Temos então as seguintes alternativas:

(a)- Se $\omega(p)$ (respectivamente $\alpha(p)$ ) contém apenas pontos regulares, então $\omega(p)$ (respectivamente $\alpha(p))$ é uma órbita periódica;

(b)- Se $\omega(p)$ (respectivamente $\alpha(p)$ ) contém pontos regulares e singulares, então $\omega(p)$ (respectivamente $\alpha(p)$ ) consiste de um conjunto de órbitas, cada uma das quais tende a um desses pontos singulares quando $t \rightarrow \pm \infty$;

(c)- Se $\omega(p)$ (respectivamente $\alpha(p))$ não contém pontos regulares, então $\omega(p)$ (respectivamente $\alpha(p))$ é um ponto singular.

Demonstração: A demonstração deste teorema pode ser encontrada em [CL55]. Em [Sot79] encontra-se uma demonstração para o caso em que $X$ é um campo de classe $C^{1}$.

\subsection{Aplicação de transição}

Primeiro vamos mostrar como podemos definir uma aplicação de transição entre duas seções transversais distintas e vamos calcular a derivada dessa aplicação para o caso de campos de vetores planares. Tais aplicações são úteis pois nos permitem definir uma aplicação de retorno para as órbitas periódicas dos campos em $\mathfrak{X}_{\Gamma, \Delta}$.

Observação 2.2 Sejam $\vec{v}=\left(v_{1}, v_{2}\right)$ e $\vec{w}=\left(w_{1}, w_{2}\right)$ dois vetores em $\mathbb{R}^{2}$. Usaremos a seguinte notação

$$
(\vec{v} \mid \vec{w})=\left(\begin{array}{ll}
v_{1} & w_{1} \\
v_{2} & w_{2}
\end{array}\right)
$$


para representar a matriz em $\mathbb{M}_{2}(\mathbb{R})$ cujas colunas são os vetores $\vec{v}$ e $\vec{w}$.

Proposição 2.1 Seja $X$ um campo de vetores de classe $C^{1}$ definido no plano $\mathbb{R}^{2}$. Dado um ponto $p_{0} \in \mathbb{R}^{2}$, denotaremos por $\varphi\left(t, p_{0}\right)$ a órbita de $X$ tal que $\varphi\left(0, p_{0}\right)=p_{0}$ e vamos supor que $T_{0} \in \mathbb{R}$ é tal que $\varphi\left(T_{0}, p_{0}\right)=p_{1}$. Sejam $\Sigma_{0}$ e $\Sigma_{1}$ seções transversais de $X$ passando pelos pontos $p_{0}$ e $p_{1}$ respectivamente. Se $\sigma_{0}: I_{0} \rightarrow \mathbb{R}^{2}$ e $\sigma_{1}: I_{1} \rightarrow \mathbb{R}^{2}$ são as respectivas parametrizações de $\Sigma_{0}$ e $\Sigma_{1}$ com $\sigma_{0}\left(s_{0}\right)=p_{0}$ e $\sigma_{1}\left(s_{1}\right)=p_{1}$, então existe uma vizinhança $U$ de $p_{0}$ e duas funções diferenciáveis

$$
\tau: U \rightarrow \mathbb{R} \quad \text { e } \quad \rho: U \rightarrow I_{1}
$$

de forma que $\tau\left(p_{0}\right)=T_{0}, \rho\left(p_{0}\right)=s_{1} e$

$$
\varphi(\tau(p), p)=\sigma_{1}(\rho(p)), \quad \forall p \in U
$$

Demonstração: Considere a função $F: \mathscr{D} \times I_{1} \subset \mathbb{R}^{4} \rightarrow \mathbb{R}^{2}$ definida por

$$
F(p, t, s)=\varphi(t, p)-\sigma_{1}(s)
$$

Como $X$ é de classe $C^{1}$, o fluxo $\varphi$ também é de classe $C^{1}$. Além do mais, como $\Sigma_{1}$ é uma seção transversal, então $F$ é uma função diferenciável que satisfaz

$$
F\left(p_{0}, T_{0}, s_{1}\right)=\varphi\left(T_{0}, p_{0}\right)-\sigma_{1}\left(s_{1}\right)=p_{1}-p_{1}=0
$$

Observe que as derivadas de $F$ com relação as variáveis $t$ e $s$ calculadas em $\left(p_{0}, T_{0}, s_{1}\right)$ são

$$
\begin{aligned}
\frac{\partial}{\partial t} F\left(p_{0}, T_{0}, s_{1}\right) & =\frac{\partial}{\partial t} \varphi\left(T_{0}, p_{0}\right)=X\left(\varphi\left(T_{0}, p_{0}\right)\right)=X\left(p_{1}\right), \\
\frac{\partial}{\partial s} F\left(p_{0}, T_{0}, s_{1}\right) & =-\sigma_{1}^{\prime}\left(s_{1}\right),
\end{aligned}
$$

resultando que a matriz

$$
D_{(t, s)} F\left(p_{0}, T_{0}, s_{1}\right)=\left(\frac{\partial}{\partial t} F\left(p_{0}, T_{0}, s_{1}\right) \mid \frac{\partial}{\partial s} F\left(p_{0}, T_{0}, s_{1}\right)\right)
$$

é não singular, isto é, det $D_{(t, s)} F\left(p_{0}, T_{0}, s_{1}\right) \neq 0$. Isso ocorre pois $\Sigma_{1}$ é uma seção transversal de $X$ parametrizada por $\sigma_{1}$.

Aplicando o teorema da função implícita em $F$ deduzimos a existência de uma vizinhança $U$ de $p$ e de duas funções diferenciáveis 


$$
\tau: U \rightarrow \mathbb{R} \quad \text { e } \quad \rho: U \rightarrow I_{1}
$$

tais que $\tau\left(p_{0}\right)=T_{0}$ e $\rho\left(p_{0}\right)=s_{1}$ com

$$
F(p, \tau(p), \rho(p))=0, \quad \forall p \in U
$$

Daí concluímos

$$
\varphi(\tau(p), p)=\sigma_{1}(\rho(p)), \quad \forall p \in U
$$

Como queríamos.

Da relação 2.2 segue que o fluxo de $X$ transforma pontos próximos de $p_{0}$ em pontos da seção transversal $\Sigma_{1}$, o que nos permite definir a aplicação

$$
\begin{aligned}
\xi: U & \longrightarrow \Sigma_{1} \\
p & \longmapsto \xi(p)=\varphi(\tau(p), p)
\end{aligned}
$$

que pode ser escrita como

$$
\xi=\sigma_{1} \circ \rho .
$$

A partir das funções $\xi$ e $\tau$ podemos definir uma aplicação $\pi$ entre as seções transversais $\Sigma_{0}$ e $\Sigma_{1}$, e uma função real $\tilde{\tau}$ definida para pontos de $\Sigma_{0}$ próximos de $p_{0}$.

Definição 2.1 Nas condições da Proposição 2.1, definimos a aplicação de transição $\pi$ do campo de vetores $X$ entre as seções transversais $\Sigma_{0}$ e $\Sigma_{1}$ como a restrição de $\xi$ à intersecção $\Sigma_{0} \cap U$, ou seja

$$
\pi: \Sigma_{0} \cap U \rightarrow \Sigma_{1}, \quad \text { onde } \quad \pi(p)=\xi(p) .
$$

Além disso, definiremos a função $\tilde{\tau}$ do sistema $X$, que exprime o tempo necessário para que um ponto $p$ de $\Sigma_{0}$ chegue a $\Sigma_{1}$ através do fluxo de $X$ pela primeira vez, como

$$
\tilde{\tau}: \Sigma_{0} \cap U \rightarrow \mathbb{R}, \quad \text { onde } \quad \tilde{\tau}(p)=\tau(p) .
$$

Ficam portanto definidas as aplicações $\pi$ e $\tilde{\tau}$ entre duas seções transversais do sistema $X$. Observe que, pelo fato de todas as funções envolvidas na construção serem diferenciáveis, essas aplicações também são diferenciáveis. 
Observação 2.3 Considere $X \in \mathfrak{X}_{\Gamma, \Delta}$ e suponha que $\gamma$ é uma órbita que intersecta a grade $[\Gamma, \Delta]$ nos pontos $\gamma\left(t_{0}\right)=p_{0}$ e $\gamma\left(t_{1}\right)=p_{1}$ transversalmente e disjunta das esquinas da grade. Suponha ainda que $\gamma(t)$ está contida no interior de uma célula $\left.\Omega_{i j}, \forall t \in\right] t_{0}, t_{1}[$. Então existem $L_{0}$ e $L_{1}$ segmentos da grade $[\Gamma, \Delta]$ contendo $p_{0}$ e $p_{1}$ respectivamente e, pela observação 2.1, existe $\delta>0$ tal que $\Sigma_{0}=L_{0} \times B_{\delta}(X)$ e $\Sigma_{1}=L_{1} \times B_{\delta}(X)$ são seções transversais para o campo

$$
\Lambda(p, Y)=(Y(p), 0)
$$

Podemos assim definir uma aplicação de transição $\Pi: \Sigma_{0} \rightarrow \Sigma_{1}$ que tem a seguinte forma

$$
\Pi(p, Y)=(\pi(p, Y), Y),
$$

onde a função $\pi(p, Y)$ nos dá a aplicação de transição entre as seções $L_{0}$ e $L_{1}$ para todo campo $Y \in B_{\delta}(X)$.

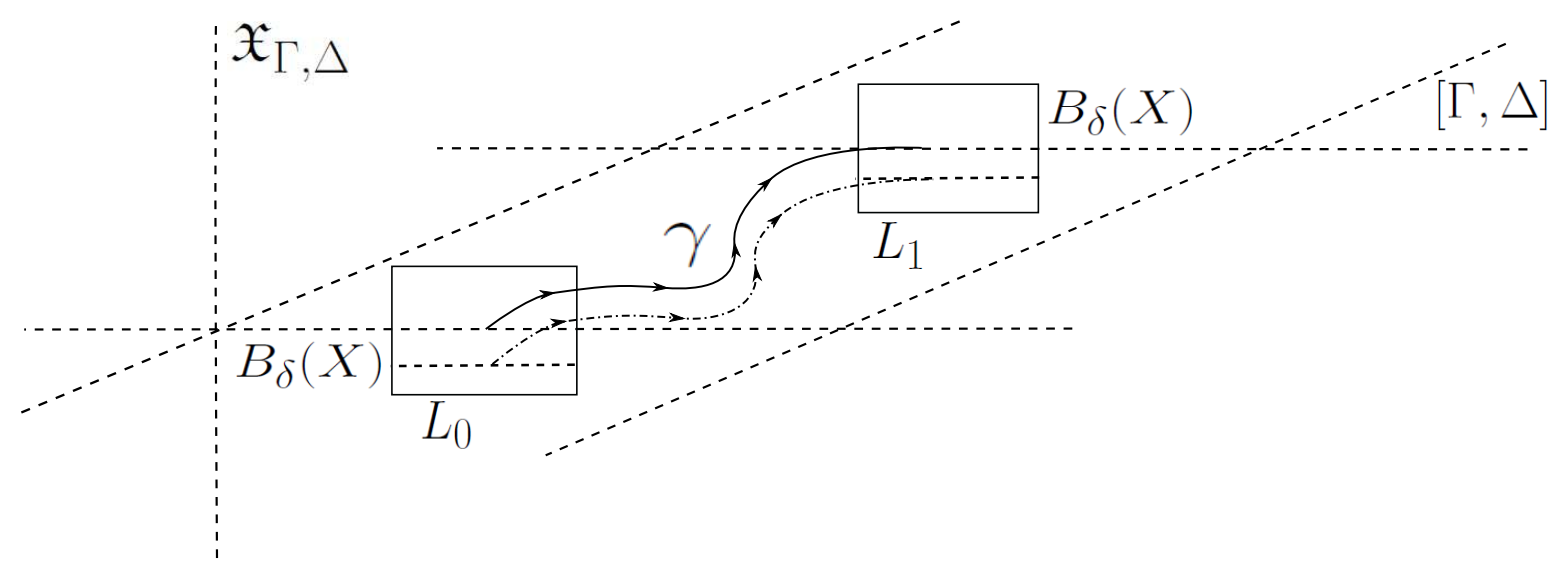

Figura 2.1: Aplicação de transição $\Pi$ entre as seções $\Sigma_{0}$ e $\Sigma_{1}$

Vamos agora nos concentrar na obtenção da derivada da aplicação de transição entre duas seções transversais associadas a um campo de vetores no plano.

Para calcular a derivada vamos usar as coordenadas locais das parametrizações $\sigma_{0} \mathrm{e}$ $\sigma_{1}$ das seções transversais $\Sigma_{0}$ e $\Sigma_{1}$, representadas respectivamente por $s$ e $\widehat{s}$.

Com as funções dadas na Definição 2.1, podemos estabelecer o seguinte diagrama comutativo

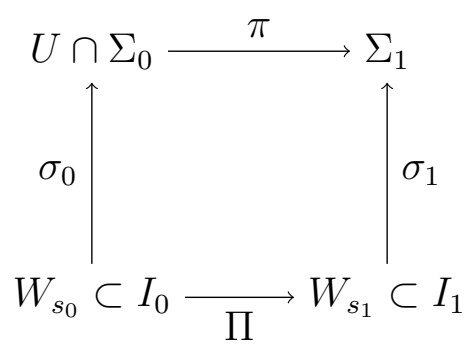

onde a aplicação $\Pi$ está definida em uma vizinhança $W_{s_{0}}$ do ponto $s_{0}$ por $\Pi=\sigma_{1}^{-1} \circ \pi \circ \sigma_{0}$, com valores em uma vizinhança $W_{s_{1}}$ do ponto $s_{1}$. 
Notemos que do Teorema da Função Implícita deduzimos que as funções $\xi, \pi, \tau$, $\tilde{\tau}$ e $\Pi$ são de classe $C^{1}$ em seus respectivos domínios de definição.

Por outro lado utilizando 2.3 podemos escrever $\Pi=\rho \circ \sigma_{0}$, e assim

$$
\frac{d}{d s} \Pi(s)=\nabla \rho\left(\sigma_{0}(s)\right) \sigma_{0}^{\prime}(s)
$$

De forma análoga podemos definir a aplicação $T=\tilde{\tau} \circ \sigma_{0}$, de classe $C^{1}$ em $W_{s_{0}}$, e obtemos

$$
\frac{d}{d s} T(s)=\nabla \tilde{\tau}\left(\sigma_{0}(s)\right) \sigma_{0}^{\prime}(s)
$$

Defina agora as matrizes

$$
M_{\Sigma_{0}}=\left(X\left(p_{0}\right) \mid-\sigma_{0}^{\prime}\left(s_{0}\right)\right) \quad M_{\Sigma_{1}}=\left(X\left(p_{1}\right) \mid-\sigma_{1}^{\prime}\left(s_{1}\right)\right) .
$$

Temos então a seguinte proposição sobre o comportamento dessas funções

Proposição 2.2 Nas condições da Proposição 2.1, se denotarmos por A a matriz

$$
A=\left(\begin{array}{ll}
1 & T^{\prime}\left(s_{0}\right) \\
0 & \Pi^{\prime}\left(s_{0}\right)
\end{array}\right)
$$

então é satisfeita a relação

$$
M_{\Sigma_{1}} A=D_{p} \varphi\left(T_{0}, p_{0}\right) M_{\Sigma_{0}} .
$$

Demonstração: Diferenciando a relação 2.1 com respeito a $p$, usando $\tilde{\tau}$ ao invés de $\tau$, e que $\frac{\partial \varphi}{\partial t}(\tilde{\tau}(p), p)=X(\varphi(\tilde{\tau}(p), p))$ resulta

$$
X(\varphi(\tilde{\tau}(p), p)) \nabla \tilde{\tau}(p)+D_{p} \varphi(\tilde{\tau}(p), p)=\sigma_{1}^{\prime}(\rho(p)) \nabla \rho(p)
$$

Substituindo na expressão $p$ por $p_{0}$, obtemos

$$
X\left(p_{1}\right) \nabla \tilde{\tau}\left(p_{0}\right)+D_{p} \varphi\left(T_{0}, p_{0}\right)=\sigma_{1}^{\prime}\left(\rho\left(p_{0}\right)\right) \nabla \rho\left(p_{0}\right)
$$

onde foram usadas as identidades $\tilde{\tau}\left(p_{0}\right)=\tau\left(p_{0}\right)=T_{0}$ e $\varphi\left(T_{0}, p_{0}\right)=p_{1}$.

Multiplicando os dois lados da igualdade 2.8 por $\sigma_{0}^{\prime}\left(s_{0}\right)$ obtemos

$$
X\left(p_{1}\right) \nabla \tilde{\tau}\left(p_{0}\right) \sigma_{0}^{\prime}\left(s_{0}\right)+D_{p} \varphi\left(T_{0}, p_{0}\right) \sigma_{0}^{\prime}\left(s_{0}\right)=\sigma_{1}^{\prime}\left(\rho\left(p_{0}\right)\right) \nabla \rho\left(p_{0}\right) \sigma_{0}^{\prime}\left(s_{0}\right)
$$

e utilizando as relações 2.4 e 2.5 temos que 


$$
X\left(p_{1}\right) T^{\prime}\left(p_{0}\right)+D_{p} \varphi\left(T_{0}, p_{0}\right) \sigma_{0}^{\prime}\left(s_{0}\right)=\sigma_{1}^{\prime}\left(s_{1}\right) \Pi^{\prime}\left(s_{0}\right) .
$$

Agora fazendo $A=\left(\begin{array}{cc}1 & T^{\prime}\left(s_{0}\right) \\ 0 & \Pi^{\prime}\left(s_{0}\right)\end{array}\right)$ decorre

$$
\begin{aligned}
M_{\Sigma_{1}} A & =\left(X\left(p_{1}\right) \mid-\sigma_{1}^{\prime}\left(s_{1}\right)\right)\left(\begin{array}{cc}
1 & T^{\prime}\left(s_{0}\right) \\
0 & \Pi^{\prime}\left(s_{0}\right)
\end{array}\right)= \\
& =\left(X\left(p_{1}\right) \mid X\left(p_{1}\right) T^{\prime}\left(p_{0}\right)-\sigma_{1}^{\prime}\left(s_{1}\right) \Pi^{\prime}\left(s_{0}\right)\right)= \\
& =\left(X\left(p_{1}\right) \mid-D_{p} \varphi\left(T_{0}, p_{0}\right) \sigma_{0}^{\prime}\left(s_{0}\right)\right) .
\end{aligned}
$$

Usando a seguinte igualdade

$$
X\left(p_{1}\right)=D_{p} \varphi\left(T_{0}, p_{0}\right) X\left(p_{0}\right)
$$

finalmente deduzimos a relação que queríamos

$$
\begin{aligned}
M_{\Sigma_{1}} A & =\left(D_{p} \varphi\left(T_{0}, p_{0}\right) X\left(p_{0}\right) \mid-D_{p} \varphi\left(T_{0}, p_{0}\right) \sigma_{0}^{\prime}\left(s_{0}\right)\right)= \\
& =D_{p} \varphi\left(T_{0}, p_{0}\right)\left(X\left(p_{0}\right) \mid-\sigma_{0}^{\prime}\left(s_{0}\right)\right)=D_{p} \varphi\left(T_{0}, p_{0}\right) M_{\Sigma_{0}} .
\end{aligned}
$$

\subsubsection{Derivada da aplicação de transição}

Com a proposição anterior podemos calcular a derivada da aplicação de transição entre duas seções transversais de um campo de vetores no plano.

Proposição 2.3 Seja $X$ um campo de vetores de classe $C^{1}$ definido no plano $\mathbb{R}^{2}$. Dado um ponto $p_{0} \in \mathbb{R}^{2}$, denotaremos por $\varphi\left(t, p_{0}\right)$ a órbita de $X$ tal que $\varphi\left(0, p_{0}\right)=p_{0}$ e vamos supor que $T_{0} \in \mathbb{R}$ é tal que $\varphi\left(T_{0}, p_{0}\right)=p_{1}$. Sejam $\Sigma_{0}$ e $\Sigma_{1}$ seções transversais de $X$ passando pelos pontos $p_{0}$ e $p_{1}$ respectivamente. Se $\sigma_{0}: I_{0} \rightarrow \mathbb{R}^{2}$ e $\sigma_{1}: I_{1} \rightarrow \mathbb{R}^{2}$ são as respectivas parametrizações de $\Sigma_{0}$ e $\Sigma_{1}$ com $\sigma_{0}\left(s_{0}\right)=p_{0}$ e $\sigma_{1}\left(s_{1}\right)=p_{1}$, então a derivada da aplicação de transição $\pi: \Sigma_{0} \rightarrow \Sigma_{1}$ no ponto $p_{0}$, definida pelo fluxo de X, é dada por: 


$$
\pi^{\prime}\left(p_{0}\right)=\frac{\operatorname{det}\left(X\left(p_{0}\right) \mid \sigma_{0}^{\prime}\left(s_{0}\right)\right)}{\operatorname{det}\left(X\left(p_{1}\right) \mid \sigma_{1}^{\prime}\left(s_{1}\right)\right)} \exp \left(\int_{0}^{T_{0}} \operatorname{div} X\left(\varphi\left(t, p_{0}\right)\right) d t\right)
$$

Demonstração: Primeiro observemos que as matrizes $M_{\Sigma_{0}}$ e $M_{\Sigma_{1}}$ são escritas da seguinte forma

$$
M_{\Sigma_{0}}=\left(X\left(p_{0}\right) \mid-\sigma_{0}^{\prime}\left(s_{0}\right)\right) \quad M_{\Sigma_{1}}=\left(X\left(p_{1}\right) \mid-\sigma_{1}^{\prime}\left(s_{1}\right)\right)
$$

e da proposição 2.2 obtemos

$$
\left(X\left(p_{1}\right) \mid-\sigma_{1}^{\prime}\left(s_{1}\right)\right)\left(\begin{array}{ll}
1 & T^{\prime}\left(s_{0}\right) \\
0 & \Pi^{\prime}\left(s_{0}\right)
\end{array}\right)=D_{p} \varphi\left(T_{0}, p_{0}\right)\left(X\left(p_{0}\right) \mid-\sigma_{0}^{\prime}\left(s_{0}\right)\right) .
$$

Calculando o determinante dos dois lados da igualdade anterior encontramos

$$
\operatorname{det}\left(X\left(p_{1}\right) \mid-\sigma_{1}^{\prime}\left(s_{1}\right)\right) \Pi^{\prime}\left(s_{0}\right)=\operatorname{det}\left(D_{p} \varphi\left(T_{0}, p_{0}\right)\right) \operatorname{det}\left(X\left(p_{0}\right) \mid-\sigma_{0}^{\prime}\left(s_{0}\right)\right)
$$

Dado que a matriz $D_{p} \varphi\left(t, p_{0}\right)$ é uma matriz fundamental do sistema variacional $X^{\prime}=$ $D X\left(\varphi\left(t, p_{0}\right)\right) X$, com $D_{p} \varphi\left(0, p_{0}\right)=I d$, pela Fórmula de Liouville vale

$$
\operatorname{det}\left(D_{p} \varphi\left(T_{0}, p_{0}\right)\right)=\exp \left(\int_{0}^{T_{0}} \operatorname{div} X\left(\varphi\left(t, p_{0}\right)\right) d t\right)
$$

Como $\Sigma_{1}$ é uma seção transversal do sistema $X$, então a matriz $M_{\Sigma_{1}}$ é não singular, e portanto $\operatorname{det}\left(X\left(p_{1}\right) \mid-\sigma_{1}^{\prime}\left(s_{0}\right)\right)=\operatorname{det} M_{\Sigma_{1}} \neq 0$. Assim, dividindo a relação 2.9 por $\operatorname{det} M_{\Sigma_{1}}$ chegamos a

$$
\Pi^{\prime}\left(s_{0}\right)=\pi^{\prime}\left(p_{0}\right)=\frac{\operatorname{det}\left(X\left(p_{0}\right) \mid-\sigma_{0}^{\prime}\left(s_{0}\right)\right)}{\operatorname{det}\left(X\left(p_{1}\right) \mid-\sigma_{1}^{\prime}\left(s_{1}\right)\right)} \exp \left(\int_{0}^{T_{0}} \operatorname{div} X\left(\varphi\left(t, p_{0}\right)\right) d t\right)
$$

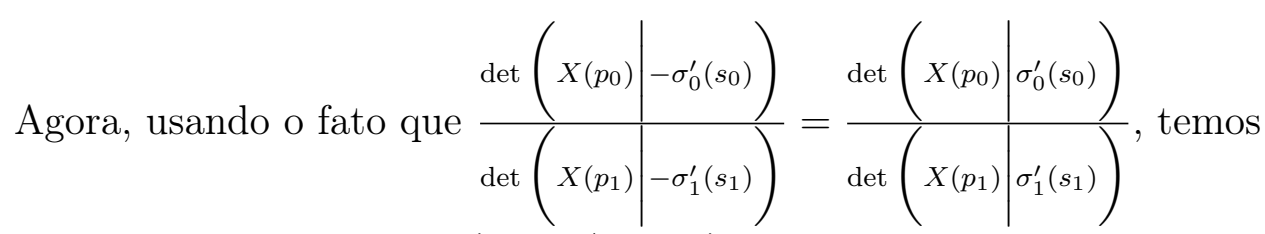

$$
\pi^{\prime}\left(p_{0}\right)=\frac{\operatorname{det}\left(X\left(p_{0}\right) \mid \sigma_{0}^{\prime}\left(s_{0}\right)\right)}{\operatorname{det}\left(X\left(p_{1}\right) \mid \sigma_{1}^{\prime}\left(s_{1}\right)\right)} \exp \left(\int_{0}^{T_{0}} \operatorname{div} X\left(\varphi\left(t, p_{0}\right)\right) d t\right) .
$$

Como queríamos. 
Podemos agora, enunciar a seguinte proposição.

Proposição 2.4 Seja $X_{\lambda}$ uma família a um parâmetro de campos de classe $C^{1}$, definidos em um aberto $U \subseteq \mathbb{R}^{2}$. Sejam $\Sigma_{0}$ e $\Sigma_{1}$ duas seções transversais para o campo $X_{\lambda_{0}}$, onde $\gamma_{0}(t)=\varphi\left(t, p_{0}, X_{\lambda_{0}}\right)$, com $\gamma_{0}(0)=p_{0} \in \Sigma_{0}$ e $\gamma_{0}\left(t_{1}\right)=p_{1} \in \Sigma_{1} . \operatorname{Seja} \pi(., \lambda): \Sigma_{0} \rightarrow \Sigma_{1} a$ aplicação de transição entre as seções $\Sigma_{0}$ e $\Sigma_{1}$ para o campo de vetores $X_{\lambda}$. Então, se $\Sigma_{1}$ é normal a $\gamma_{0}$, temos

$\frac{\partial \pi}{\partial \lambda}\left(p_{0}, \lambda_{0}\right)=\frac{\frac{\partial \pi}{\partial p}\left(p_{0}, \lambda_{0}\right)}{\left|X_{\lambda_{0}}\left(p_{0}\right)\right|} \int_{0}^{t_{1}} \exp \left(-\int_{0}^{t} \operatorname{div} X_{\lambda_{0}}\left(\gamma_{0}(u)\right) d u\right) \operatorname{det}\left(X_{\lambda_{0}}\left(\gamma_{0}(t)\right) \mid \frac{\partial X_{\lambda_{0}}}{\partial \lambda}\left(\gamma_{0}(t)\right)\right) d t$ onde $\frac{\partial \pi}{\partial p}\left(p_{0}, \lambda_{0}\right)$ é dada pela proposição 2.3.

Demonstração: A demonstração dessa proposição pode ser encontrada em [Sot81] e [ALGM73].

\subsubsection{Derivada da aplicação de retorno para órbitas periódicas}

Considere um campo de vetores $X \in \mathfrak{X}_{\Gamma, \Delta}$ e suponha que esse campo possui uma órbita periódica $\gamma$. Suponha também que $\gamma$ intersecta a grade $[\Gamma, \Delta] \operatorname{nos}$ pontos $\left\{E_{1}, E_{2}, \ldots, E_{k}\right\}$, transversalmente e de forma disjunta das esquinas da grade.

Em volta de todo ponto $E_{s}$ podemos tomar um pequeno segmento $L_{E_{s}}$ da grade $[\Gamma, \Delta]$ como uma seção transversal para $X$. Podemos então definir uma aplicação de retorno $\pi$ para a órbita $\gamma$ que consiste em compor as aplicações de transição entre as seções transversais mencionadas. A figura 2.2 ilustra o que estamos descrevendo.

Proposição 2.5 Nas condições anteriores, se a órbita $\gamma$ for tal que $\gamma\left(t_{s}\right)=E_{s}, s \in$

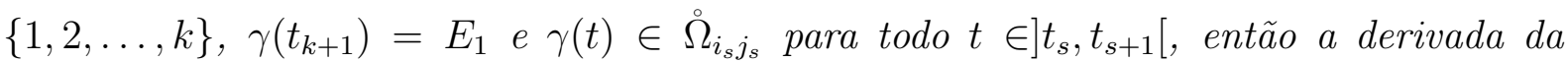
aplicação de retorno para a órbita periódica $\gamma$ é

$$
\pi^{\prime}=\exp \left(\sum_{s=1}^{k}\left(t_{s+1}-t_{s}\right) \operatorname{tr} A_{i_{s} j_{s}}\right)
$$

Demonstração: Seja $X \in \mathfrak{X}_{\Gamma, \Delta}$. É fácil verificar que se $\gamma(t)$ permanece em uma célula $\Omega_{i j}$ para $t \in\left[t_{0}, t_{1}\right]$, então 


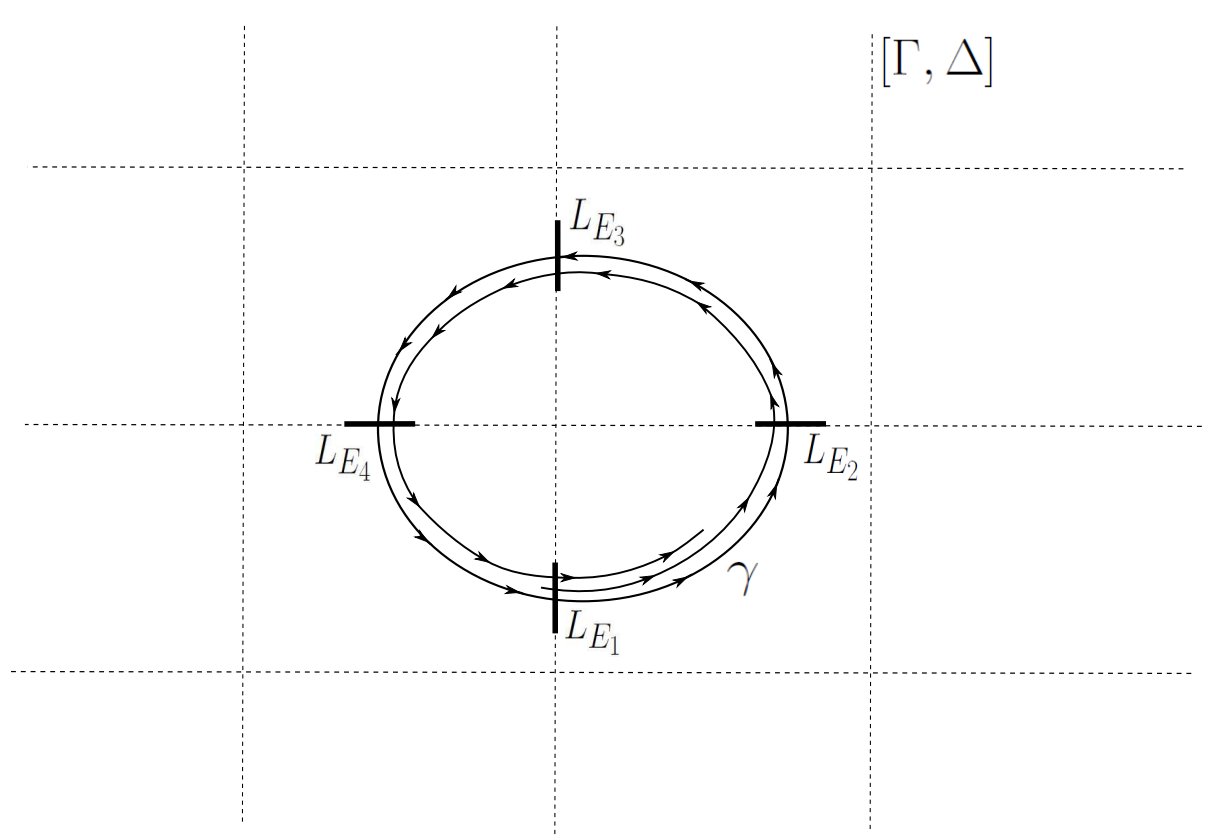

Figura 2.2: Aplicação de retorno para a órbita $\gamma$

$$
\int_{t_{0}}^{t_{1}} \operatorname{div} X(\gamma(t)) d t=\left(t_{1}-t_{0}\right) \operatorname{tr} A_{i j}
$$

onde $\left.X\right|_{\Omega_{i j}}=A_{i j} p+b_{i j}$.

Considere $T_{s}, s \in\{1,2, \ldots, k\}$ as aplicações de transição entre as seções transversais $L_{E_{s}}$, parametrizadas por $\sigma_{s}: I_{s} \rightarrow L_{E_{s}}$, com $\sigma_{s}\left(u_{s}\right)=E_{s}$. Derivando a aplicação de retorno

$$
\pi=T_{k} \circ \ldots \circ T_{1}
$$

e usando 2.3, encontramos

$$
\pi^{\prime}=\prod_{s=1}^{k} \frac{\operatorname{det}\left(X\left(E_{s}\right) \mid-\sigma_{s}^{\prime}\left(u_{s}\right)\right)}{\operatorname{det}\left(X\left(E_{s+1}\right) \mid-\sigma_{s+1}^{\prime}\left(u_{s+1}\right)\right)} \exp \left(\int_{t_{s}}^{t_{s+1}} \operatorname{div} X(\gamma(t)) d t\right)
$$

Observe agora que

$$
\prod_{s=1}^{k} \frac{\operatorname{det}\left(X\left(E_{s}\right) \mid-\sigma_{s}^{\prime}\left(u_{s}\right)\right)}{\operatorname{det}\left(X\left(E_{s+1}\right) \mid-\sigma_{s+1}^{\prime}\left(u_{s+1}\right)\right)}=1
$$

Logo, usando 2.10, concluímos que

$$
\pi^{\prime}=\exp \left(\sum_{s=1}^{k}\left(t_{s+1}-t_{s}\right) \operatorname{tr} A_{i_{s} j_{s}}\right) .
$$




\subsection{Compactificação de Poincaré}

Seja $\gamma$ uma órbita do campo de vetores $X \in \mathfrak{X}_{\Gamma, \Delta}$ tal que a semi-órbita $\gamma^{+}$(respectivamente $\gamma^{-}$) está contida em um compacto. O teorema de Poincaré-Bendixson descreve os possíveis conjuntos $\omega$-limite (respectivamente $\alpha$-limite) da semi-órbita $\gamma^{+}$(respectivamente $\gamma^{-}$). Falta, portanto, descrevermos o comportamento assintótico das semi-órbitas que não estão contidas em compactos, ou seja, o comportamento de órbitas com pontos limite no infinito.

O estudo das órbitas próximo do infinito pode ser feito através de uma técnica denominada compactificação de Poincaré. Ela recebe esse nome em homenagem ao matemático francês Henri Poincaré, que aplicou a técnica, no estudo de campos de vetores polinomiais.

Começamos definindo em $\mathbb{R}^{3}$ os seguintes conjuntos

$$
\begin{gathered}
\mathbb{S}^{2}=\left\{\left(x_{1}, x_{2}, x_{3}\right) \in \mathbb{R}^{3} \mid x_{1}^{2}+x_{2}^{2}+x_{3}^{2}=1\right\}, \\
\mathbb{S}^{1}=\left\{\left(x_{1}, x_{2}, x_{3}\right) \in \mathbb{S}^{2} \mid x_{3}=0\right\}, \\
\mathbb{H}_{+}=\left\{\left(x_{1}, x_{2}, x_{3}\right) \in \mathbb{S}^{2} \mid x_{3}>0,\right. \\
\mathbb{H}_{-}=\left\{\left(x_{1}, x_{2}, x_{3}\right) \in \mathbb{S}^{2} \mid x_{3}<0,\right. \\
\mathcal{N}=\left\{\left(x_{1}, x_{2}, x_{3}\right) \in \mathbb{R}^{3} \mid x_{3}=1\right\} .
\end{gathered}
$$

Em $\mathbb{R}^{3}, \mathbb{S}^{2}$ é a esfera de raio 1 , sendo $\mathbb{S}^{1}$ seu equador. O plano $\mathcal{N}$ é um plano tangente a $\mathbb{S}^{2}$ no ponto $(0,0,1)$ que chamamos de pólo norte. Chamamos $\mathbb{H}_{+}$de hemisfério norte ou hemisfério superior e $\mathbb{H}_{-}$de hemisfério sul ou hemisfério inferior.

Se $r$ é uma reta que passa pela origem $(0,0,0)$ e por um ponto $p_{0} \in \mathcal{N}$, então ela intercepta a esfera $\mathbb{S}^{2}$ em dois pontos distintos $p_{0}^{+}$e $p_{0}^{-}$, o primeiro no hemisfério superior e o segundo no hemisfério inferior.

Isto nos dá dois difeomorfismos sobrejetores $f_{+}: \mathcal{N} \rightarrow \mathbb{H}_{+}$e $f_{-}: \mathcal{N} \rightarrow \mathbb{H}_{-}$cujas expressões são

$$
f_{+}\left(x_{1}, x_{2}\right)=\frac{\left(x_{1}, x_{2}, 1\right)}{\left(x_{1}^{2}+x_{2}^{2}+1\right)^{\frac{1}{2}}}
$$




$$
f_{-}\left(x_{1}, x_{2}\right)=-\frac{\left(x_{1}, x_{2}, 1\right)}{\left(x_{1}^{2}+x_{2}^{2}+1\right)^{\frac{1}{2}}} .
$$

As aplicações $f_{+}$e $f_{-}$são as projeções centrais sobre $\mathbb{H}_{+}$e $\mathbb{H}_{-}$do plano $\mathcal{N}$.

Se $X$ é um campo de vetores em $\mathbb{R}^{2}$, os difeomorfismos $f_{-}$e $f_{+}$induzem em $\mathbb{H}_{-} \cup \mathbb{H}_{+}$ um campo de vetores $\tilde{X}$ da seguinte maneira:

$$
\tilde{X}\left(y_{1}, y_{2}, y_{3}\right)=\left\{\begin{array}{lll}
D f_{+}\left(x_{1}, x_{2}\right) X\left(x_{1}, x_{2}\right) & \text { se } & \left(y_{1}, y_{2}, y_{3}\right)=f_{+}\left(x_{1}, x_{2}\right) \in \mathbb{H}_{+} \\
D f_{-}\left(x_{1}, x_{2}\right) X\left(x_{1}, x_{2}\right) & \text { se } & \left(y_{1}, y_{2}, y_{3}\right)=f_{-}\left(x_{1}, x_{2}\right) \in \mathbb{H}_{-}
\end{array}\right.
$$

Analisar o comportamento assintótico das órbitas não limitadas de $X$ será útil estender $\tilde{X}$ para o equador $\mathbb{S}^{1}$, obtendo assim um campo em toda a esfera $\mathbb{S}^{2}$. O estudo deste campo numa vizinhança do equador fornecerá informações importantes sobre as órbitas não limitadas do sistema.

Nem sempre isso é possível, mas devido ao fato dos campos em $\mathfrak{X}_{\Gamma, \Delta}$ serem lineares por partes conseguiremos fazer essa extensão.

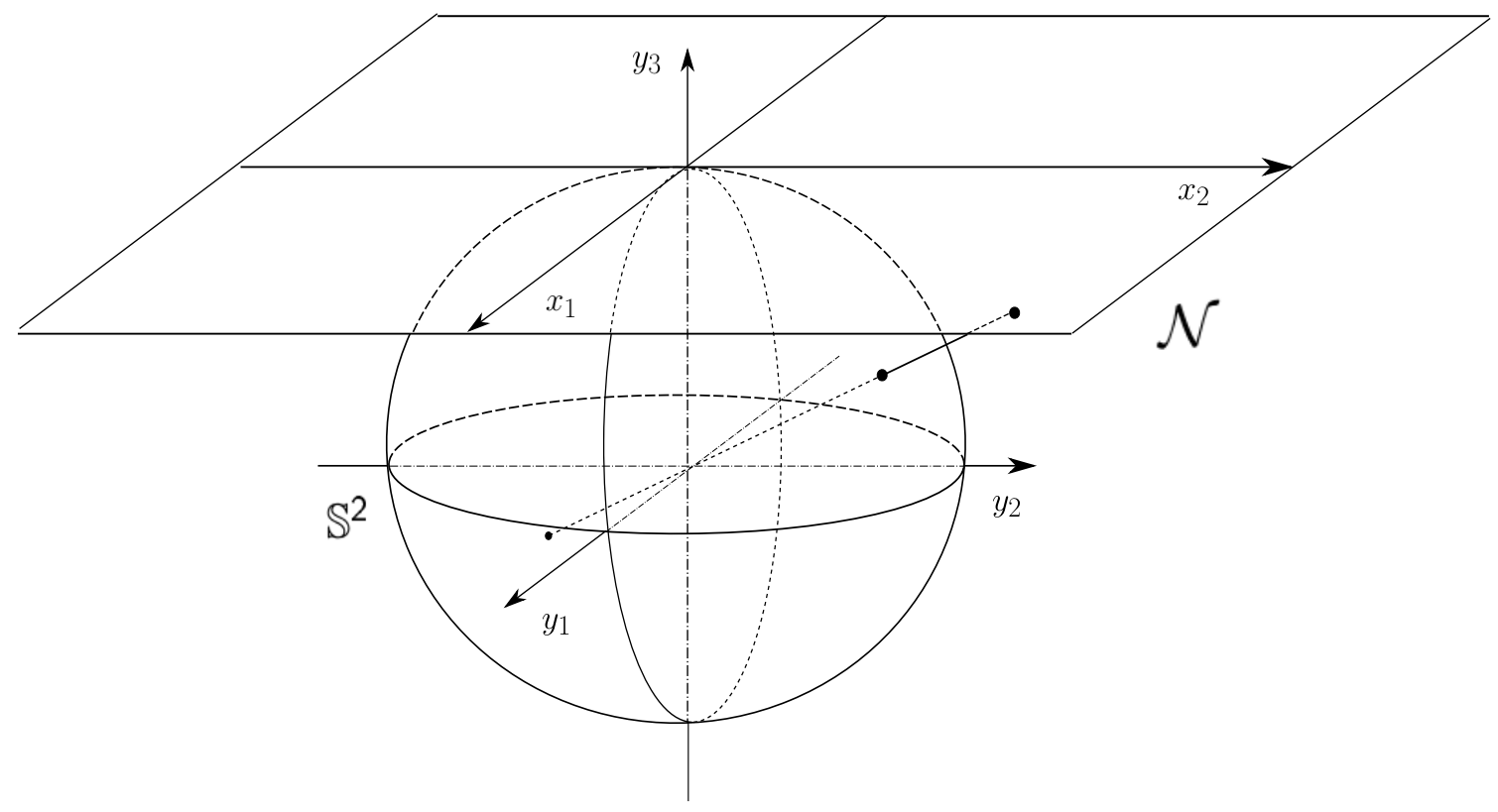

Figura 2.3: Compactificação de Poincaré

Proposição 2.6 Seja $X \in \mathfrak{X}_{\Gamma, \Delta}$. Considere $\tilde{X}$ o campo induzido em $\mathbb{S}^{2} \backslash \mathbb{S}^{1}=\mathbb{H}_{-} \cup \mathbb{H}_{+}$ definido como anteriormente. Então $\tilde{X}$ pode ser estendido a um campo de vetores tangente $a \mathbb{S}^{1}$ e analitico por partes em $\mathbb{S}^{2}$.

Demonstração: Para demonstrarmos a proposição vamos calcular o campo $\tilde{X}$ em um sistema de coordenadas conveniente de $\mathbb{S}^{2}$. 
Sejam $\Phi_{i}: U_{i} \rightarrow \mathbb{R}^{2}, \Psi_{i}: V_{i} \rightarrow \mathbb{R}^{2}, i=1,2,3$. Onde:

$$
\begin{aligned}
& U_{i}=\left\{\left(y_{1}, y_{2}, y_{3}\right) \in \mathbb{S}^{2} / y_{i}>0\right\}, \\
& V_{i}=\left\{\left(y_{1}, y_{2}, y_{3}\right) \in \mathbb{S}^{2} / y_{i}<0\right\} .
\end{aligned}
$$

$\left\{\begin{array}{l}\Phi_{i}\left(y_{1}, y_{2}, y_{3}\right)=\frac{1}{y_{i}}\left(y_{j}, y_{k}\right), \quad \forall\left(y_{1}, y_{2}, y_{3}\right) \in U_{i} ; \quad i, j, k=1,2,3, \quad j<k, \quad i \neq j, k, \\ \Psi_{i}\left(y_{1}, y_{2}, y_{3}\right)=\frac{1}{y_{i}}\left(y_{j}, y_{k}\right), \quad \forall\left(y_{1}, y_{2}, y_{3}\right) \in V_{i} ; \quad i, j, k=1,2,3, \quad j<k, \quad i \neq j, k .\end{array}\right.$

Seja $\left(y_{1}, y_{2}, y_{3}\right) \in U_{1} \cap \mathbb{H}_{+}$. Então $\left(y_{1}, y_{2}, y_{3}\right)=f_{+}\left(x_{1}, x_{2}\right) \mathrm{e}$

$$
\left(\Phi_{1} \circ f_{+}\right)\left(x_{1}, x_{2}\right)=\left\{\begin{array}{l}
z_{1}=\frac{x_{2}}{x_{1}}=\frac{y_{2}}{y_{1}} \\
z_{2}=\frac{1}{x_{1}}=\frac{y_{3}}{y_{1}}
\end{array}\right.
$$

Note ainda que

$$
D\left(\Phi_{1} \circ f_{+}\right)\left(x_{1}, x_{2}\right)=\left(\begin{array}{cc}
-\frac{x_{2}}{x_{1}^{2}} & \frac{1}{x_{1}} \\
-\frac{1}{x_{1}^{2}} & 0
\end{array}\right)
$$

donde

$$
\begin{array}{r}
D \Phi_{1}\left(y_{1}, y_{2}, y_{3}\right) \circ \tilde{X}\left(y_{1}, y_{2}, y_{3}\right)=D\left(\Phi_{1} \circ f_{+}\right)\left(x_{1}, x_{2}\right) \circ X\left(x_{1}, x_{2}\right)= \\
=\frac{1}{x_{1}^{2}}\left(-x_{2} P\left(x_{1}, x_{2}\right)+x_{1} Q\left(x_{1}, x_{2}\right),-P\left(x_{1}, x_{2}\right)\right) .
\end{array}
$$

Esta é a expressão de $\tilde{X}$ em $U_{1} \cap \mathbb{H}_{+}$. Colocando a expressão em função de $z_{1}, z_{2}$ obtemos:

$$
z_{2}\left(-z_{1} P\left(\frac{1}{z_{2}}, \frac{z_{1}}{z_{2}}\right)+Q\left(\frac{1}{z_{2}}, \frac{z_{1}}{z_{2}}\right),-z_{2} P\left(\frac{1}{z_{2}}, \frac{z_{1}}{z_{2}}\right)\right) .
$$

Repetindo o processo para $y \in U_{1} \cap \mathbb{H}_{-}$obtemos a mesma expressão 2.12 .

Como $P$ e $Q$ são funções lineares por partes, então restrito a cada célula da grade eles são polinômios de grau menor ou igual a 1. Logo 2.12 é a expressão de um campo analítico por partes que estende $\tilde{X}$ em $U_{1}$.

Verifica-se analogamente que as expressões de $\tilde{X}$ em $U_{2}$ e $U_{3}$ são respectivamente:

$$
z_{2}\left(P\left(\frac{z_{1}}{z_{2}}, \frac{1}{z_{2}}\right)-z_{1} Q\left(\frac{z_{1}}{z_{2}}, \frac{1}{z_{2}}\right),-z_{2} Q\left(\frac{z_{1}}{z_{2}}, \frac{1}{z_{2}}\right)\right)
$$




$$
\left(P\left(z_{1}, z_{2}\right), Q\left(z_{1}, z_{2}\right)\right) \text {. }
$$

Por outro lado, as expressões de $\tilde{X}$ em $V_{1}, V_{2}$ e $V_{3}$ são, respectivamente, as mesmas expressões 2.12, 2.13 e 2.14. Elas são todas compatíveis e analíticas por partes.

Dessa forma, fica claro que podemos estender o campo $\tilde{X}$ para toda a esfera $\mathbb{S}^{2}$. E, pelas expressões encontradas, no interior das imagens das células da grade esse campo é analítico.

Falta agora apenas mostrarmos que $\mathbb{S}^{1}$ é invariante pelo campo estendido.

Seja $y \in \mathbb{S}^{1} \cap U_{1}$. Logo $y_{3}=0$, então por 2.11 temos $z_{2}=\frac{y_{3}}{y_{1}}=0$. Sendo assim a expressão do campo em y é dada por 2.12, cuja segunda componente é nula. Do mesmo modo é nula a segunda componente do campo em $\mathbb{S}^{1} \cap V_{1}$.

Como o equador nas coordenadas $\left(z_{1}, z_{2}\right)$ está identificado com $z_{2}=0$, concluímos portanto que $\mathbb{S}^{1}$ é invariante por $\tilde{X}$.

Assim denotemos por $X_{\mathbb{S}^{2}}$ o campo analítico por partes que estende $\tilde{X}$ para toda a esfera. Definimos então o campo $X_{\mathbb{S}^{2}}$ como a compactificação de Poincaré de $X$ na esfera $\mathbb{S}^{2}$.

Observe que, quando restringimos $X_{\mathbb{S}^{2}}$ ao hemisfério superior $\mathbb{H}_{+}$, então pela forma como a compactificação foi construída vale

$$
X_{\mathbb{S}^{2}}\left(f_{+}\left(x_{1}, x_{2}\right)\right)=D f_{+}\left(x_{1}, x_{2}\right) X\left(x_{1}, x_{2}\right)
$$

Daí concluímos que os campos $X$ e $\left.X_{\mathbb{S}^{2}}\right|_{\mathbb{H}_{+}}$são diferenciavelmente conjugados, sendo $f_{+}$a conjugação. O mesmo vale quando consideramos $\mathbb{H}_{-}$e $f_{-}$.

Pela observação anterior, vemos que em $\mathbb{S}^{2}$, temos duas cópias de $X$, uma no hemisfério superior e outra no hemisfério inferior.

Observe agora que se $r$ é uma reta que passa pelo ponto $(0,0,-1)$ e intersecta a esfera em outro ponto $p_{0}$, então ela intercepta o plano $\mathcal{N}$ em um ponto $p_{0}^{\mathcal{N}}$. Isso nos dá um difeomorfismo sobrejetor $g: \mathbb{S}^{2} \backslash(0,0,-1) \rightarrow \pi$, cuja expressão é

$$
g\left(y_{1}, y_{2}, y_{3}\right)=\left(\frac{2 y_{1}}{y_{3}+1}, \frac{2 y_{2}}{y_{3}+1}\right)
$$

Podemos então induzir um campo de vetores $\hat{X}$ em $\mathbb{R}^{2}$ por

$$
\hat{X}\left(x_{1}, x_{2}\right)=D g\left(y_{1}, y_{2}, y_{3}\right) X_{\mathbb{S}^{2}}\left(y_{1}, y_{2}, y_{3}\right)
$$

onde $g\left(y_{1}, y_{2}, y_{3}\right)=\left(x_{1}, x_{2}\right)$.

Pelas mesmas observações feitas anteriormente, o campo $X_{\mathbb{S}^{2}}$ restrito a $\mathbb{S}^{2} \backslash(0,0,-1)$ 
e o campo $\hat{X}$ são diferenciavelmente conjugados, sendo $g$ a conjugação.

Então, se tomarmos o conjunto $\mathbb{D}=\left\{\left(x_{1}, x_{2}\right) \in \mathbb{R}^{2} \mid x_{1}^{2}+x_{2}^{2}=2\right\}$, o campo original $X$ e o campo $\hat{X}$ restrito a $\mathbb{D}$ são diferenciavelmente conjugados, sendo a conjugação $g \circ f_{+}$.

Por essas observações, definimos então o campo $X_{\mathbb{D}}=\left.\hat{X}\right|_{\mathbb{D}}$ como a compactificação de Poincaré de $X$ no disco $\mathbb{D}$.

Como $X_{\mathbb{D}}$ e $X_{\mathbb{S}^{2}}$ são diferenciavelmente conjugados inclusive nos conjuntos $\mathbb{S}^{1}$ e $\partial \mathbb{D}$, quando estivermos analisando esses campos no infinito, faremos os cálculos usando a compactificação que nos for mais apropriada.

Com a notação introduzida anteriormente, dizemos que uma singularidade é simples se no ponto de singularidade o campo é suave e o determinante da matriz jacobiana no ponto é não nulo, se além disso as partes reais de seus autovalores forem todas não nulas dizemos então que a singularidade é hiperbólica. Se o determinante do jacobiano nesse ponto for positivo, dizemos que o ponto é uma anti-sela, e se o determinante for negativo, dizemos que o ponto é uma sela.

Chamaremos de singularidades finitas as singularidades de $X_{\mathbb{S}^{2}}$, que não estão em $\mathbb{S}^{1}$, as singularidades em $\mathbb{S}^{1}$ serão chamadas de singularidades infinitas.

Da mesma forma uma órbita periódica será finita se não possui pontos em $\mathbb{S}^{1} \mathrm{e}$, lembrando que, como o equador é invariante por $X_{\mathbb{S}^{2}}$, quando $\mathbb{S}^{1}$ for uma órbita periódica diremos que ela é a órbita periódica no infinito.

Na figura 2.5 , temos a representação da imagem da grade $[\Gamma, \Delta]$ pela função $f_{+}$. Iremos nos referir a esse conjunto em $\mathbb{S}^{2}$ ou $\mathbb{D}$ como a grade compactificada.

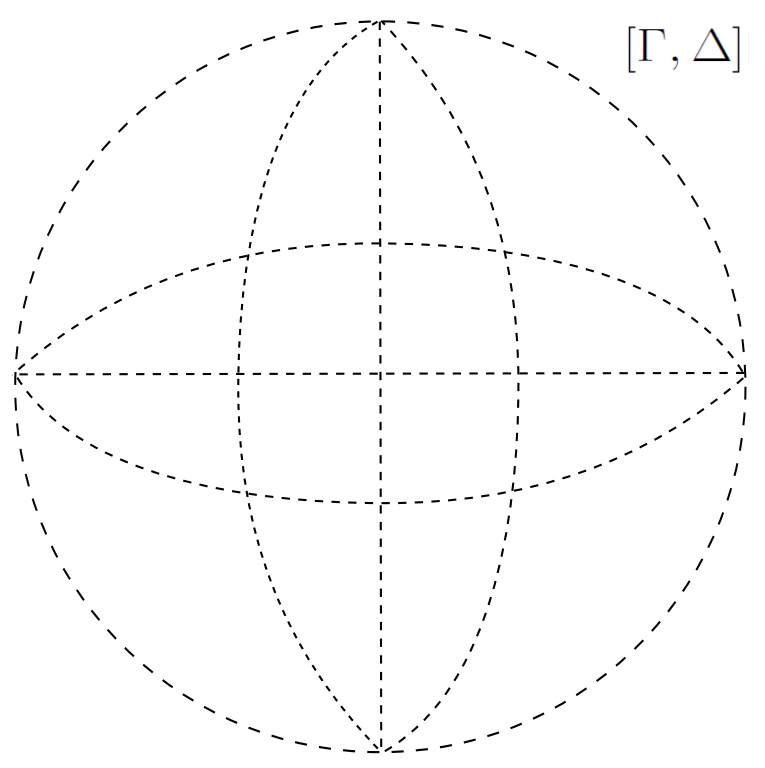

Figura 2.4: Grade compactificada 


\subsection{Comportamento dos campos no infinito via com- pactificação}

Analisaremos agora com detalhes o comportamento da compactificação dos campos de $\mathfrak{X}_{\Gamma, \Delta}$ no infinito.

Como veremos mais adiante, é de fundamental importância entendermos a relação entre as singularidades hiperbólicas no infinito e o campo de vetores $X$.

Dado um campo $X \in \mathfrak{X}_{\Gamma, \Delta}$, e analisando as expressões de $X_{\mathbb{S}^{2}}$, é fácil vermos que o campo de vetores $X_{\mathbb{S}^{2}}$ é determinado em

$$
\mathbb{S}^{1} \backslash\{(1,0,0),(-1,0,0),(0,1,0),(0,-1,0)\}
$$

pela expressão dos campos lineares não homogêneos nas células infinitas $\Omega_{00}, \Omega_{0 m}, \Omega_{n 0} \mathrm{e}$ $\Omega_{n m}$. A esse respeito provaremos a seguinte proposição.

Proposição 2.7 Considere o campo de vetores linear não homogêneo

$$
X(x, y)=A\left(\begin{array}{l}
x \\
y
\end{array}\right)+\vec{b}
$$

então para a compactificação de Poincaré $X_{\mathbb{S}^{2}}$ vale

(a)- $S e(\operatorname{tr} A)^{2}-4 \operatorname{det} A<0$, então $X_{\mathbb{S}^{2}}$ não possui singularidade no infinito;

(b)- $S e(\operatorname{tr} A)^{2}-4 \operatorname{det} A=0$ e $\operatorname{tr} A \neq 0$, então ou todo o equador $\mathbb{S}^{1}$ é composto por pontos de equilíbrio, ou $X_{\mathbb{S}^{2}}$ possui duas singularidades não hiperbólicas no infinito;

(c)- $S e(\operatorname{tr} A)^{2}-4 \operatorname{det} A>0$ e $\operatorname{det} A<0$, então $X_{\mathbb{S}^{2}}$ possui quatro singularidade no infinito, todas elas são nós hiperbólicos;

(d)- $S e(\operatorname{tr} A)^{2}-4 \operatorname{det} A>0$ e $\operatorname{det} A>0$, então $X_{\mathbb{S}^{2}}$ possui quatro singularidades no infinito, duas delas são nós hiperbólicos e duas são selas. As órbitas de $X$ que estão contidas na reta do auto espaço forte de $X$ são separatrizes das selas no infinito;

(e)- $S e(\operatorname{tr} A)^{2}-4 \operatorname{det} A>0 e \operatorname{det} A=0$, então $X_{\mathbb{S}^{2}}$ possui quatro singularidades não hiperbólicas no infinito. Pode existir uma reta de singularidades finitas que conecta duas singularidades infinitas;

(f)- Se $\operatorname{tr} A=0$ e $\operatorname{det} A=0$, então $X_{\mathbb{S}^{2}}$ possui duas singularidades não hiperbólicas no infinito. Pode existir uma reta de singularidades finitas que conecta as singularidades infinitas. 
Demonstração: É fácil verificarmos que as singularidade de $X_{\mathbb{S}^{2}}$ ocorrem aos pares e são antípodas em relação a origem. Além disso, se

$$
A=\left(\begin{array}{ll}
a_{11} & a_{12} \\
a_{21} & a_{22}
\end{array}\right) \text { e } \vec{b}=\left(\begin{array}{l}
b_{1} \\
b_{2}
\end{array}\right)
$$

então $\left(z_{1}, 0\right) \in \mathbb{S}^{1} \cap\left(U_{1} \cup V_{1}\right)$ é uma singularidade no infinito se $z_{1}$ for uma solução real de

$$
-a_{12} z_{1}^{2}+\left(a_{22}-a_{11}\right) z_{1}+a_{21}=0
$$

De forma análoga, $\left(z_{1}, 0\right) \in \mathbb{S}^{1} \cap\left(U_{2} \cup V_{2}\right)$ é uma singularidade no infinito se $z_{1}$ é uma solução real de

$$
-a_{21} z_{1}^{2}+\left(a_{11}-a_{22}\right) z_{1}+a_{12}=0
$$

Para as equações de segundo grau 2.15 e 2.16 encontramos $\Delta=(\operatorname{tr} A)^{2}-4 \operatorname{det} A$.

(a) Se $\Delta<0$, então 2.15 e 2.16 não possuem soluções reais. Portanto $X_{\mathbb{S}^{2}}$ não possui singularidades no infinito.

Considere então $\Delta \geqslant 0$. Daí as soluções da equação 2.15 são

$$
z_{1}=\frac{\left(a_{22}-a_{11}\right) \pm \sqrt{\Delta}}{2 a_{12}}
$$

e da equaçao 2.16 são

$$
z_{1}=\frac{\left(a_{11}-a_{22}\right) \pm \sqrt{\Delta}}{2 a_{21}} .
$$

Se $\left(z_{1}, 0\right) \in \mathbb{S}^{1} \cap\left(U_{1} \cup V_{1}\right)$ a diferencial do campo nesse ponto é

$$
\left(\begin{array}{cc}
-2 a_{12} z_{1}+\left(a_{22}-a_{11}\right) & b_{2}-z_{1} b_{1} \\
0 & -a_{11}-a_{12} z_{1}
\end{array}\right)
$$

e se $\left(z_{1}, 0\right) \in \mathbb{S}^{1} \cap\left(U_{2} \cup V_{2}\right)$ então a diferencial do campo nesse ponto é

$$
\left(\begin{array}{cc}
-2 a_{21} z_{1}+\left(a_{11}-a_{22}\right) & b_{1}-z_{1} b_{2} \\
0 & -a_{22}-a_{21} z_{1}
\end{array}\right)
$$

Sem perda de generalidade, iremos supor a partir de agora que a matriz $A$ está escrita na forma canônica de Jordan.

(b) Se $\Delta=0$ e $\operatorname{tr} A \neq 0$, precisamos considerar dois casos.

Se $A$ for diagonalizável, então as expressões 2.15 e 2.16 se anulam para todo $z_{1}$, logo todo ponto em $X_{\mathbb{S}^{2}}$ é singularidade. 
Agora se $A$ não for diagonalizável, podemos escrever

$$
A=\left(\begin{array}{ll}
\lambda & 1 \\
0 & \lambda
\end{array}\right)
$$

onde $\lambda$ é o autovalor de $A$. Logo 2.15 é

$$
-z_{1}^{2}=0
$$

e a diferencial do campo nesse ponto fica

$$
\left(\begin{array}{cc}
0 & b_{2} \\
0 & -\lambda
\end{array}\right)
$$

e portanto o ponto não é uma singularidade hiperbólica.

Além disso a expressão de 2.16 nunca se anula, e portanto $X_{\mathbb{S}^{2}}$ não possui singularidades em $\mathbb{S}^{1} \cap\left(U_{2} \cup V_{2}\right)$.

(c) Se $\Delta>0$ e $\operatorname{det} A<0$, podemos escrever

$$
A=\left(\begin{array}{cc}
\lambda_{1} & 0 \\
0 & \lambda_{2}
\end{array}\right)
$$

onde $\lambda_{1} \lambda_{2}<0$. Assim para 2.15 temos

$$
\left(\lambda_{2}-\lambda_{1}\right) z_{1}=0
$$

com a diferencial

$$
\left(\begin{array}{cc}
\lambda_{2}-\lambda_{1} & b_{2} \\
0 & -\lambda_{1}
\end{array}\right)
$$

e portanto o ponto é um nó hiperbólico, atrator se $\lambda_{1}>0$ e repulsor se $\lambda_{1}<0$.

Para 2.16 temos

$$
\left(\lambda_{1}-\lambda_{2}\right) z_{1}=0
$$

com a diferencial

$$
\left(\begin{array}{cc}
\lambda_{1}-\lambda_{2} & b_{1} \\
0 & -\lambda_{2}
\end{array}\right)
$$

e portanto o ponto é um nó hiperbólico, atrator se $\lambda_{2}>0$ e repulsor se $\lambda_{2}<0$.

Assim, concluímos que $X_{\mathbb{S}^{2}}$ possui quatro nós hiperbólicos no infinito.

(d) Considere agora que $\Delta>0$ e $\operatorname{det} A>0$, podemos escrever

$$
A=\left(\begin{array}{cc}
\lambda_{1} & 0 \\
0 & \lambda_{2}
\end{array}\right)
$$


com $\lambda_{1} \lambda_{2}>0$. Vamos supor que $\left|\lambda_{1}\right|>\left|\lambda_{2}\right|$, o outro caso pode ser tratado de forma semelhante.

Assim para 2.15 temos

$$
\left(\lambda_{2}-\lambda_{1}\right) z_{1}=0
$$

com a diferencial

$$
\left(\begin{array}{cc}
\lambda_{2}-\lambda_{1} & b_{2} \\
0 & -\lambda_{1}
\end{array}\right) .
$$

Analisando as possibilidades para o sinal de $\lambda_{1}$, concluímos que $\lambda_{2}-\lambda_{1}$ e $-\lambda_{1}$ possuem sinais iguais, logo o ponto é um nó hiperbólico no infinito, atrator se $\lambda_{1}>0$ e repulsor se $\lambda_{1}<0$.

Para 2.16 temos

$$
\left(\lambda_{1}-\lambda_{2}\right) z_{1}=0
$$

com a diferencial

$$
\left(\begin{array}{cc}
\lambda_{1}-\lambda_{2} & b_{1} \\
0 & -\lambda_{2}
\end{array}\right) .
$$

Novamente analisando as possibilidades para o sinal de $\lambda_{2}$, concluímos que $\lambda_{1}-\lambda_{2}$ e $-\lambda_{2}$ possuem sinais opostos, logo o ponto no infinito é uma sela hiperbólica.

Além disso, vemos facilmente que as órbitas de $X$ que estão contidas na reta do auto espaço forte de $A$ tendem para os pontos de sela no infinito quando o tempo cresce ou decresce. Logo, concluímos que a imagem dessas órbitas por $f_{+}$são separatrizes das selas no infinito.

(e) Se $\Delta>0$ e $\operatorname{det} A=0$, podemos escrever

$$
A=\left(\begin{array}{ll}
0 & 0 \\
0 & \lambda
\end{array}\right)
$$

Assim para 2.15 temos

$$
\lambda z_{1}=0
$$

com a diferencial

$$
\left(\begin{array}{cc}
\lambda & b_{2} \\
0 & 0
\end{array}\right)
$$

e portanto o ponto não é uma singularidade hiperbólica infinita.

Para 2.16 temos

$$
-\lambda z_{1}=0
$$

com a diferencial

$$
\left(\begin{array}{cc}
-\lambda & b_{1} \\
0 & 0
\end{array}\right)
$$

e o ponto não é uma singularidade hiperbólica no infinito. 
Além disso, se $b_{1} \neq 0, X$ não possui singularidades finitas, e se $b_{1}=0$, a reta $y=-\frac{b_{2}}{\lambda}$ é uma linha de singularidades. Essa reta de pontos singulares tende para as singularidades infinitas em $\mathbb{S}^{1} \cap\left(U_{1} \cup V_{1}\right)$.

(f) $\operatorname{Se} \operatorname{tr} A=0 \mathrm{e} \operatorname{det} A=0$, podemos escrever

$$
A=\left(\begin{array}{ll}
0 & 1 \\
0 & 0
\end{array}\right)
$$

Assim para 2.15 temos

$$
-z_{1}^{2}=0
$$

com a diferencial

$$
\left(\begin{array}{cc}
0 & b_{2} \\
0 & 0
\end{array}\right) .
$$

Logo o ponto não é uma singularidade hiperbólica no infinito.

Além disso, 2.16 nunca se anula, e portanto $X_{\mathbb{S}^{2}}$ não possui singularidades infinitas em $\mathbb{S}^{1} \cap\left(U_{2} \cup V_{2}\right)$.

Se $b_{2} \neq 0, X$ não possui singularidades finitas, e se $b_{2}=0$, então a reta $y=-b_{1}$ é uma linha de singularidades. Essa reta de pontos singulares tende para as singularidades infinitas de $X$.

Observe que, para uma matriz $A$ sem estar na forma canônica de Jordan, os valores de $z_{1}$ que são singularidades hiperbólicas no infinito são os coeficiente angulares das retas que contém os auto-espaços da matriz $A$. Logo, como as singularidades hiperbólicas de $X_{\mathbb{S}^{2}}$ estão associadas com as direções dos auto-espaços de $A$, e eles estão contidos em retas, podemos então em cada quadrante de $\mathbb{S}^{1}$ possuir no máximo duas singularidades infinitas hiperbólicas.

E como, para os campos em $\mathfrak{X}_{\Gamma, \Delta}$, as singularidades no infinito no interior de cada quadrante são determinadas por sistemas lineares não homogêneos, concluímos que tais campos podem possuir no máximo oito singularidades hiperbólicas em

$$
\mathbb{S}^{1} \backslash\{(1,0,0),(-1,0,0),(0,1,0),(0,-1,0)\}
$$

Introduziremos agora um outro sistema de coordenadas que facilitará nossos cálculos quando formos analisar o comportamento de $X_{\mathbb{S}^{2}}$ no infinito.

Considere a aplicação $S: \mathbb{R} \times \mathbb{R}_{+} \rightarrow \mathbb{S}_{+}^{2} \backslash(0,0,1)$ dada por

$$
S(\theta, \rho)=\frac{(\cos \theta, \operatorname{sen} \theta, \rho)}{\sqrt{\rho^{2}+1}} .
$$

Observe que $S$ é um recobrimento universal de $\mathbb{S}_{+}^{2} \backslash(0,0,1)$. Podemos então reescrever o campo $X_{\mathbb{S}^{2}}$ nas coordenadas $(\theta, \rho)$. 


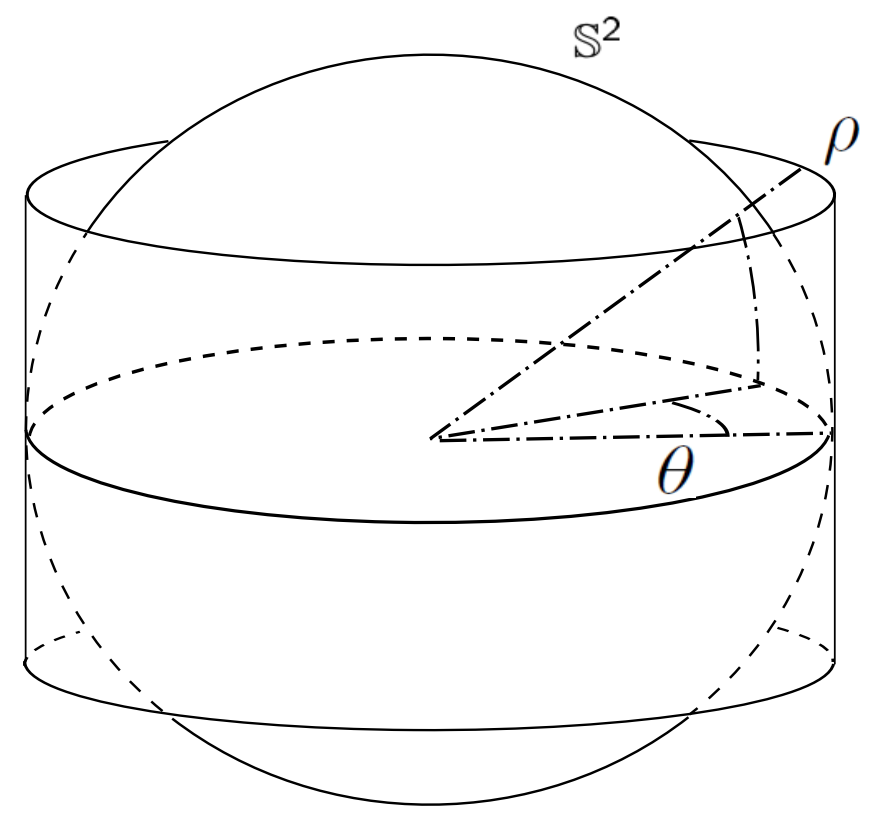

Figura 2.5: Mudança de coordenadas próximo do infinito

Lema 2.1 Nas coordenadas $(\theta, \rho)$ o campo de vetores $X_{\mathbb{S}^{2}}$ tem a seguinte expressão:

$$
\left(A_{1}(\theta, \rho)+\rho A_{0}(\theta)\right) \frac{\partial}{\partial \theta}-\rho\left(R_{1}(\theta, \rho)+\rho R_{0}(\theta)\right) \frac{\partial}{\partial \rho}
$$

onde

$$
\begin{gathered}
A_{0}(\theta)=a_{2} \cos \theta-a_{1} \operatorname{sen} \theta, \quad R_{0}(\theta)=a_{1} \cos \theta+a_{2} \operatorname{sen} \theta \\
A_{1}(\theta, \rho)=-\operatorname{sen} \theta\left(b_{11} \cos \theta+b_{12} \operatorname{sen} \theta+\sum_{i=1}^{n} c_{1}^{i}\left|\cos \theta-\rho \gamma_{i}\right|+\sum_{j=1}^{m} d_{1}^{j}\left|\operatorname{sen} \theta-\rho \delta_{j}\right|\right. \\
+\cos \theta\left(b_{21} \cos \theta+b_{22} \operatorname{sen} \theta+\sum_{i=1}^{n} c_{2}^{i}\left|\cos \theta-\rho \gamma_{i}\right|+\sum_{j=1}^{m} d_{2}^{j}\left|\operatorname{sen} \theta-\rho \delta_{j}\right|\right), \\
R_{1}(\theta, \rho)=\cos \theta\left(b_{11} \cos \theta+b_{12} \operatorname{sen} \theta+\sum_{i=1}^{n} c_{1}^{i}\left|\cos \theta-\rho \gamma_{i}\right|+\sum_{j=1}^{m} d_{1}^{j}\left|\operatorname{sen} \theta-\rho \delta_{j}\right|\right) \\
+\operatorname{sen} \theta\left(b_{21} \cos \theta+b_{22} \operatorname{sen} \theta+\sum_{i=1}^{n} c_{2}^{i}\left|\cos \theta-\rho \gamma_{i}\right|+\sum_{j=1}^{m} d_{2}^{j}\left|\operatorname{sen} \theta-\rho \delta_{j}\right|\right) .
\end{gathered}
$$

Demonstração: Levando em conta que as componentes $P$ e $Q$ são dadas pela equação 2.1, a prova decorre diferenciando as relações $\rho x=\cos \theta, \rho y=\operatorname{sen} \theta$ entre as coordenadas e substituindo $P$ e $Q$ nas expressões encontradas. Assim basta simplificar as expressões 


$$
\left\{\begin{array}{l}
\theta^{\prime}=\rho\left(-\operatorname{sen} \theta P\left(\frac{\cos \theta}{\rho}, \frac{\operatorname{sen} \theta}{\rho}\right)+\cos \theta Q\left(\frac{\cos \theta}{\rho}, \frac{\operatorname{sen} \theta}{\rho}\right)\right), \\
\rho^{\prime}=\rho^{2}\left(-\cos \theta P\left(\frac{\cos \theta}{\rho}, \frac{\operatorname{sen} \theta}{\rho}\right)-\operatorname{sen} \theta Q\left(\frac{\cos \theta}{\rho}, \frac{\operatorname{sen} \theta}{\rho}\right)\right) .
\end{array}\right.
$$

Nessas coordenadas é mais simples analisarmos as condições para que o campo se anule em algum ponto do infinito, pois o equador é representado nas coordenadas $(\theta, \rho)$ pelos pontos $\rho=0$ em $\mathbb{R} \times \mathbb{R}_{+}$. Assim para um ponto $p_{0} \in \mathbb{S}^{1} \cap \mathbb{S}^{2}$ ser uma singularidade de $X_{\mathbb{S}^{2}}$ deve existir $\theta_{0}$ tal que $A_{1}\left(\theta_{0}, 0\right)=0$. Temos então o seguinte lema:

Lema 2.2 As singularidades de $X_{\mathbb{S}^{2}}$ ao longo do equador $\mathbb{S}^{1}$ são dadas pelos zeros da componente angular $A_{1}(\theta, 0)$ da seguinte forma.

No $k$-quadrante, $k \in\{1,2,3,4\}$, são as soluções reais da equação $A_{1 k}(\theta)=0$ no intervalo $\left[(k-1) \frac{\pi}{2}, k \frac{\pi}{2}\right]$, onde:

$$
\begin{aligned}
& A_{11}(\theta)=\left[b_{21}+c_{2}\right] \cos ^{2} \theta+\left[b_{22}-b_{11}+d_{2}-c_{1}\right] \operatorname{sen} \theta \cos \theta-\left[b_{12}+d_{1}\right] \operatorname{sen}^{2} \theta, \\
& A_{12}(\theta)=\left[b_{21}-c_{2}\right] \cos ^{2} \theta+\left[b_{22}-b_{11}+d_{2}+c_{1}\right] \operatorname{sen} \theta \cos \theta-\left[b_{12}+d_{1}\right] \operatorname{sen}^{2} \theta, \\
& A_{13}(\theta)=\left[b_{21}-c_{2}\right] \cos ^{2} \theta+\left[b_{22}-b_{11}-d_{2}+c_{1}\right] \operatorname{sen} \theta \cos \theta-\left[b_{12}-d_{1}\right] \operatorname{sen}^{2} \theta, \\
& A_{14}(\theta)=\left[b_{21}+c_{2}\right] \cos ^{2} \theta+\left[b_{22}-b_{11}-d_{2}-c_{1}\right] \operatorname{sen} \theta \cos \theta-\left[b_{12}-d_{1}\right] \operatorname{sen}^{2} \theta .
\end{aligned}
$$

com

$$
c_{1}=\sum_{i=1}^{n} c_{1}^{i}, \quad c_{2}=\sum_{i=1}^{n} c_{2}^{i}, \quad d_{1}=\sum_{j=1}^{m} d_{1}^{j}, \quad d_{2}=\sum_{j=1}^{m} d_{2}^{j} .
$$

Demonstração: A demonstração é consequência direta de 2.17, calculada em pontos da forma $(\theta, 0)$.

Com as notações introduzidas anteriormente vale o seguinte corolário.

Corolário 2.1 Uma singularidade no infinito está na direção do eixo $x$ se e somente se:

$$
\left(b_{21}+c_{2}\right)\left(-b_{21}+c_{2}\right)=0 \text {. }
$$


E uma singularidade no infinito está na direção do eixo y se e somente se:

$$
\left(b_{12}+d_{1}\right)\left(-b_{12}+d_{1}\right)=0 .
$$

Demonstração: Basta observar que uma singularidade na direção do eixo $x$ acontece quando $A_{11}(0)=A_{14}(0)=0$ ou quando $A_{12}(\pi)=A_{13}(\pi)=0$, ou seja devemos ter $b_{21}+c_{2}=0 \mathrm{ou}-b_{21}+c_{2}=0$. O mesmo vale para as singularidades na direção do eixo y, calculando as funções em $\frac{\pi}{2}$ e $\frac{3 \pi}{2}$.

Lema 2.3 A função $R_{1}(\theta, 0)$ da componente radial do campo de vetores $X_{\mathbb{S}^{2}}$ calculada no equador $\rho=0$ tem a seguinte expressão. No $k$-quadrante, $k \in\{1,2,3,4\}$, a função é dada por:

$$
\begin{aligned}
& R_{11}(\theta)=\left[b_{11}+c_{1}\right] \cos ^{2} \theta+\left[b_{12}+b_{21}+d_{1}+c_{2}\right] \operatorname{sen} \theta \cos \theta+\left[b_{22}+d_{2}\right] \operatorname{sen}^{2} \theta, \\
& R_{12}(\theta)=\left[b_{11}-c_{1}\right] \cos ^{2} \theta+\left[b_{12}+b_{21}+d_{1}-c_{2}\right] \operatorname{sen} \theta \cos \theta+\left[b_{22}+d_{2}\right] \operatorname{sen}^{2} \theta, \\
& R_{13}(\theta)=\left[b_{11}-c_{1}\right] \cos ^{2} \theta+\left[b_{12}+b_{21}-d_{1}-c_{2}\right] \operatorname{sen} \theta \cos \theta+\left[b_{22}-d_{2}\right] \operatorname{sen}^{2} \theta, \\
& R_{14}(\theta)=\left[b_{11}+c_{1}\right] \cos ^{2} \theta+\left[b_{12}+b_{21}-d_{1}+c_{2}\right] \operatorname{sen} \theta \cos \theta+\left[b_{22}-d_{2}\right] \operatorname{sen}^{2} \theta .
\end{aligned}
$$

Demonstração: A demonstração é consequência direta de 2.18, calculada em pontos da forma $(\theta, 0)$.

Além de analisar se um ponto no infinito é uma singularidade queremos também determinar se essa singularidade é ou não hiperbólica. Logo para garantirmos que uma singularidade ao longo do equador é hiperbólica precisamos analisar as derivadas do campo $X_{\mathbb{S}}$ nas coordenadas $(\theta, \rho)$.

Dada uma singularidade no infinito $\left(\theta_{0}, 0\right)$, a linearização do campo de vetores $X_{\mathbb{S}}$ na singularidade é:

$$
D X_{\mathbb{S}}\left(\theta_{0}, 0\right)=\left(\begin{array}{cc}
\frac{\partial A_{1}\left(\theta_{0}, 0\right)}{\partial \theta} & \frac{\partial A_{1}\left(\theta_{0}, 0\right)}{\partial \rho}+A_{0}\left(\theta_{0}\right) \\
0 & -R_{1}\left(\theta_{0}, 0\right)
\end{array}\right) .
$$

Segue que a matriz da linearização em um ponto singular no infinito é sempre triangular. Portanto seus autovalores são sempre reais e iguais aos elementos na diagonal principal da matriz. Para o ponto $\left(\theta_{0}, 0\right)$ ser hiperbólico deve então valer:

$$
\operatorname{det} D X_{\mathbb{S}}\left(\theta_{0}, 0\right)=-\left(\frac{\partial A_{1}\left(\theta_{0}, 0\right)}{\partial \theta}\right)\left(R_{1}\left(\theta_{0}, 0\right)\right) \neq 0 \text {. }
$$




\subsubsection{Derivada da aplicação de retorno para a órbita no infinito}

Com as notações introduzidas na seção anterior agora podemos encontrar uma expressão para a derivada da aplicação de retorno de uma órbita no infinito.

Proposição 2.8 Suponha que o equador é uma órbita periódica do sistema $X_{\mathbb{S}}$. Podemos então definir a transformação de Poincaré $\pi$ associada à órbita no infinito, tal transformação é analítica e sua derivada é dada por:

$$
\pi^{\prime}(0)=\exp \left[\sum_{j=1}^{4} \int_{\theta_{j-1}}^{\theta_{j}}-\frac{R_{1 j}(\theta)}{A_{1 j}(\theta)} d \theta\right]
$$

onde

$$
\theta_{j}=\frac{\pi}{2} j, \quad j=0,1,2,3,4
$$

Demonstração: Observe que estamos supondo que o equador é uma órbita periódica do sistema $X_{\mathbb{S}^{2}}$. Não existem portanto singularidades no infinito e $A_{1}(\theta, 0) \neq 0, \forall \theta \in \mathbb{R}$. Além disso como o equador é uma órbita periódica, então nas coordenadas $(\theta, \rho)$ a reta $\rho=0$ é uma órbita do sistema.

Nas coordenadas $(\theta, \rho)$ a grade $[\Gamma, \Delta]$, gera curvas que são as pré-imagens das linhas $x=\gamma_{i}$ e $y=\delta_{j}$. Essas curvas são dadas pelas equações $\rho \gamma_{i}=\cos \theta$ e $\rho \delta_{j}=\operatorname{sen} \theta$.

Pelo fato do equador ser uma órbita periódica do sistema sabemos que o campo de vetores é paralelo ao eixo $\theta$ quando temos $\rho=0$.

As curvas mencionadas anteriormente cortam o eixo $\theta$ transversalmente, então podemos considerar pequenos arcos dessas curvas como seções transversais do sistema numa vizinhança do eixo $\theta$.

As curvas $\rho \gamma_{i}=\cos \theta$ cortam o eixo $\theta$ em um ângulo de coeficiente angular $-\frac{1}{\gamma_{i}}$ em $\theta=\frac{\pi}{2}$ e $\frac{1}{\gamma_{i}}$ em $\theta=\frac{3 \pi}{2}$, e as retas $\rho \delta_{j}=\operatorname{sen} \theta$ cortam o eixo $\theta$ em um ângulo de coeficiente angular $\frac{1}{\delta_{j}}$ em $\theta=0$ ou $\theta=2 \pi$ e $-\frac{1}{\delta_{j}}$ em $\theta=\pi$.

Para simplificar a notação vamos denotar as seções que estão em $\rho \gamma_{i}=\cos \theta$ por $\Sigma_{i}^{\frac{\pi}{2}}$ quando $\theta=\frac{\pi}{2}$ e $\Sigma_{i}^{\frac{3 \pi}{2}}$ quando $\theta=\frac{3 \pi}{2}$, e as seções que estão em $\rho \delta_{j}=\operatorname{sen} \theta$ serão $\Sigma_{j}^{0}$ quando $\theta=0, \Sigma_{j}^{\pi}$ quando $\theta=\pi$ e $\Sigma_{j}^{2 \pi}$ quando $\theta=2 \pi$.

Em cada célula definida pelas curvas previamente descritas, o campo de vetores é diferenciável. Podemos aplicar a proposição 2.3 para definir uma aplicação de transição entre os lados das célula.

No plano $(\theta, \rho)$ os pontos $(0,0)$ e $(2 \pi, 0)$ correspondem ao ponto $(1,0,0)$ de $\mathbb{S}^{1} \cap$ $\mathbb{S}^{2}$, enquanto os pontos $\left(\frac{\pi}{2}, 0\right),(\pi, 0)$ e $\left(\frac{3 \pi}{2}, 0\right)$ correspondem respectivamente aos pontos 
$(0,1,0),(-1,0,0)$ e $(0,-1,0)$ de $\mathbb{S}^{1} \cap \mathbb{S}^{2}$

Suponha agora que a órbita associada ao equador está orientada positivamente com relação a $\theta$. Logo analisar a estabilidade da órbita é o mesmo que analisar a derivada de uma aplicação de transição entre duas seções transversais $\Sigma_{j}^{0}$ e $\Sigma_{j}^{2 \pi}$.

Podemos então definir uma aplicação de retorno para essa órbita

$$
\pi: \Sigma_{j}^{0} \rightarrow \Sigma_{j}^{2 \pi}
$$

com

$$
\pi(p)=T_{2(n+m)} \circ \cdots \circ T_{2} \circ T_{1}(p)
$$

Onde $T_{k}(p)$ são as aplicações de transição entre seções transversais consecutivas do sistema. A figura 2.6 ilustra a situação que estamos considerando.

Como em cada célula o sistema é analítico, então as aplicações de transição $T_{k}$ são analíticas e portanto $\pi$ assim definida é analítica.

Aplicando a regra da cadeia à expressão em 2.21, encontramos que a derivada da aplicação de retorno tem a seguinte forma:

$$
\pi^{\prime}(0)=\Pi_{l=1}^{2(n+m)} T_{k}^{\prime}(0)
$$

Observe agora que, pela proposição 2.3, a derivada da aplicação de transição entre duas seções transversais quaisquer que se intersectam $\left(\theta_{0}, 0\right)$ e cortam o eixo $\theta$ transversalmente com ângulos $\alpha_{1}$ e $\alpha_{2}$ respectivamente é igual a $\operatorname{sen} \alpha_{1} / \operatorname{sen} \alpha_{2}$.

Como o equador é uma órbita, nas coordenadas $(\theta, \rho)$ a solução do sistema é escrito como $\Phi(t)=(\theta(t), 0)$, onde vale que $\theta^{\prime}(t)=A_{1}(\theta(t), 0)$.

Além disso, dada uma aplicação de transição $T$ entre duas seções transversais $\Sigma_{1}$ e $\Sigma_{2}$ que não se intersectam e que cortam o eixo $\theta$ transversalmente respectivamente em $\left(\theta_{1}, 0\right)$ e $\left(\theta_{2}, 0\right)$ com ângulos $\alpha_{1}$ e $\alpha_{2}$, onde $\theta_{2}>\theta_{1}, t_{2}>t_{1}, \theta\left(t_{1}\right)=\theta_{1}$ e $\theta\left(t_{2}\right)=\theta_{2}$, então por 2.3 a derivada de $T$ é:

$$
T^{\prime}(0)=\frac{\operatorname{det}\left(\begin{array}{cc}
A_{1}\left(\theta_{1}, 0\right) & \cos \alpha_{1} \\
0 & \operatorname{sen} \alpha_{1}
\end{array}\right)}{\operatorname{det}\left(\begin{array}{cc}
A_{1}\left(\theta_{2}, 0\right) & \cos \alpha_{2} \\
0 & \operatorname{sen} \alpha_{2}
\end{array}\right)} \exp \left[\int_{t_{1}}^{t_{2}}\left(\frac{\partial A_{1}(\theta(t), 0)}{\partial \theta}-R_{1}(\theta(t), 0)\right) d t\right] .
$$

Como $A_{1}(\theta, 0) \neq 0 \forall \theta \in \mathbb{R}$, então $\theta^{\prime}(t)=A_{1}(\theta(t), 0) \neq 0 \forall t \in \mathbb{R}$. Fazendo então a mudança de variáveis $\theta=\theta(t)$ temos: 


$$
T^{\prime}(0)=\frac{A_{1}\left(\theta_{1}, 0\right) \operatorname{sen} \alpha_{1}}{A_{1}\left(\theta_{2}, 0\right) \operatorname{sen} \alpha_{2}} \exp \left[\int_{\theta_{1}}^{\theta_{2}} \frac{\frac{\partial A_{1}(\theta, 0)}{\partial \theta}}{A_{1}(\theta, 0)}-\frac{R_{1}(\theta, 0)}{A_{1}(\theta, 0)} d \theta\right],
$$

ou seja

$$
T^{\prime}(0)=\frac{A_{1}\left(\theta_{1}, 0\right) \operatorname{sen} \alpha_{1}}{A_{1}\left(\theta_{2}, 0\right) \operatorname{sen} \alpha_{2}} \exp \left[\ln \left(\frac{A_{1}\left(\theta_{2}, 0\right)}{A_{1}\left(\theta_{1}, 0\right)}\right)+\int_{\theta_{1}}^{\theta_{2}}-\frac{R_{1}(\theta, 0)}{A_{1}(\theta, 0)} d \theta\right] .
$$

Daí concluímos

$$
T^{\prime}(0)=\frac{\operatorname{sen} \alpha_{1}}{\operatorname{sen} \alpha_{2}} \exp \left(\int_{\theta_{1}}^{\theta_{2}}-\frac{R_{1}(\theta, 0)}{A_{1}(\theta, 0)} d \theta\right)
$$

Agora nos resta observar que as aplicações de transição entre as seções consecutivas usadas na definição da aplicação de Poincaré apresentam simetria, ou seja, a primeira derivada dessas aplicações em pontos antipodais é recíproca. Assim os únicos termos que aparecem em 2.22 são os referentes as seções consecutivas em $\theta=\frac{\pi l}{2} l=0,1,2,3$.

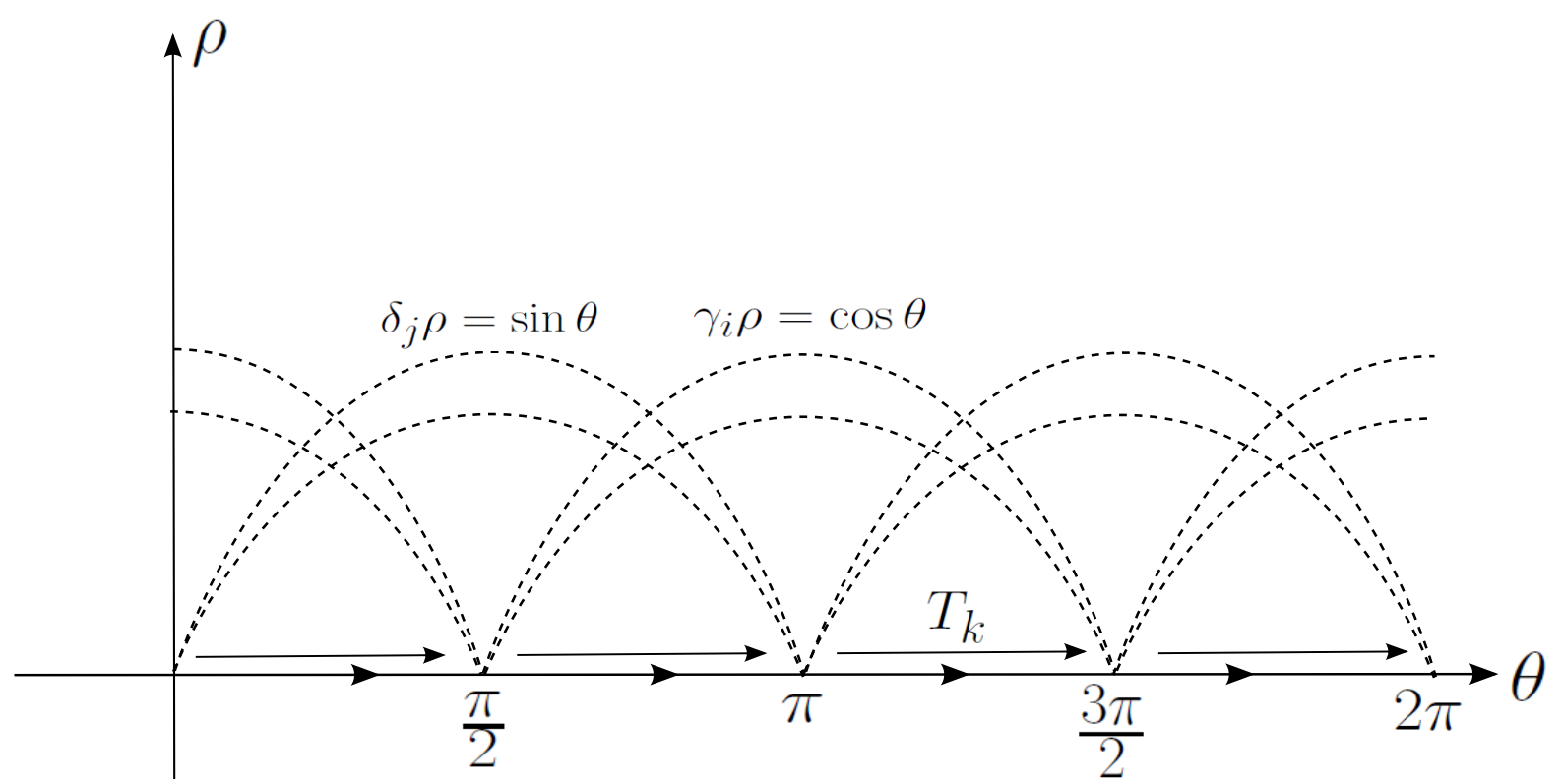

Figura 2.6: Aplicação de retorno para a órbita periódica no infinito

Enunciaremos aqui duas proposições que aparecerão mais tarde e indicam como dois tipos de perturbação afetam os pontos no infinito do campos seccionalmente lineares.

Proposição 2.9 Suponha que o equador é uma órbita periódica de $X_{\mathbb{S}^{2}}$. Considere a família a um parâmetro $X_{\nu}=X+\nu\left(x \frac{\partial}{\partial x}+y \frac{\partial}{\partial y}\right)$. Então o equador é uma órbita periódica hiperbólica de $X_{\nu \mathbb{S}^{2}}$ para todo $\nu \neq 0$ suficientemente pequeno.

Demonstração: Observe que para a família de campos a um parâmetro $X_{\nu}$ vale que 
$A_{\nu 1}(\theta, 0)=A_{1}(\theta, 0)$ e $R_{\nu 1}(\theta, 0)=\nu+R_{1}(\theta, 0)$.

Usando a expressão encontrada em 2.8 temos

$$
\pi_{\nu}^{\prime}(0)=\exp \left[\sum_{l=1}^{4} \int_{\frac{\pi(l-1)}{2}}^{\frac{\pi l}{2}}-\frac{R_{\nu 1, l}(\theta)}{A_{\nu 1, l}(\theta)} d \theta\right]
$$

e pelas relações acima

$$
\pi_{\nu}^{\prime}(0)=\exp \left[\sum_{l=1}^{4} \int_{\frac{\pi(l-1)}{2}}^{\frac{\pi l}{2}}-\frac{\nu+R_{1, l}(\theta)}{A_{1, l}(\theta)} d \theta\right] .
$$

Derivando a expressão anterior com relação a $\nu$ tem-se

$$
\frac{d}{d \nu} \pi_{\nu}^{\prime}(0)=\exp \left[\sum_{l=1}^{4} \int_{\frac{\pi(l-1)}{2}}^{\frac{\pi l}{2}}-\frac{\nu+R_{1, l}(\theta)}{A_{1, l}(\theta)} d \theta\right]\left(-\sum_{l=1}^{4} \int_{\frac{\pi(l-1)}{2}}^{\frac{\pi l}{2}} \frac{1}{A_{1, l}(\theta)} d \theta\right)
$$

ou seja

$$
\left.\frac{d}{d \nu} \pi_{\nu}^{\prime}(0)\right|_{\nu=0}=\pi^{\prime}(0)\left(-\sum_{l=1}^{4} \int_{\frac{\pi(l-1)}{2}}^{\frac{\pi l}{2}} \frac{1}{A_{1, l}(\theta)} d \theta\right) .
$$

Como estamos supondo que o equador é uma órbita periódica, então $A_{1}(\theta, 0) \neq 0$, $\forall \theta \in \mathbb{R}, \operatorname{assim} A_{1, l}(\theta) \neq 0, \forall \theta \in \mathbb{R}, l=1,2,3,4$. Por $A_{1}$ ser uma função contínua então $A_{1, l}$ possuem todas o mesmo sinal, logo concluímos que

$$
\left(-\sum_{l=1}^{4} \int_{\frac{\pi(l-1)}{2}}^{\frac{\pi l}{2}} \frac{1}{A_{1, l}(\theta)} d \theta\right) \neq 0
$$

e portanto temos $\frac{d}{d \nu} \pi_{\nu}^{\prime}(0) \neq 0$.

Assim a função $\pi_{\nu}^{\prime}(0)$ é crescente ou decrescente com relação a $\nu$ e portanto podemos escolher valores de $\nu$ suficientemente pequenos para que $\pi_{\nu}^{\prime}(0) \neq 1$.

Seja $X=(P, Q)$ um campo de vetores no plano $\mathbb{R}^{2}$. Definimos o campo $R_{\omega}(X)$ dado pela rotação do campo $X$ por um ângulo $\omega$ como sendo $R_{\omega}(X)=\left(P_{\omega}, Q_{\omega}\right)$ com

$$
\left(\begin{array}{c}
P_{\omega} \\
Q_{\omega}
\end{array}\right)=\left(\begin{array}{cc}
\cos \omega & \operatorname{sen} \omega \\
-\operatorname{sen} \omega & \cos \omega
\end{array}\right)\left(\begin{array}{l}
P \\
Q
\end{array}\right)
$$

Proposição 2.10 Seja $X \in \mathfrak{X}_{\Gamma, \Delta}$, seja $X_{\omega}$ o campo $X$ rotacionado por $\omega$, então vale as seguintes relações:

$$
\left(\begin{array}{l}
A_{\omega 1}(\theta) \\
R_{\omega 1}(\theta)
\end{array}\right)=\left(\begin{array}{cc}
\cos \omega & -\operatorname{sen} \omega \\
\operatorname{sen} \omega & \cos \omega
\end{array}\right)\left(\begin{array}{l}
A_{1}(\theta) \\
R_{1}(\theta)
\end{array}\right)
$$


Demonstração: Seja $X \in \mathfrak{X}_{\Gamma, \Delta}$, então $X(x, y)=(P(x, y), Q(x, y))$ pode ser escrito da seguinte forma:

$$
\begin{aligned}
& P(x, y)=a_{1}+b_{11} x+b_{12} y+\sum_{i=1}^{n} c_{1}^{i}\left|x-\gamma_{i}\right|+\sum_{j=1}^{m} d_{1}^{j}\left|y-\delta_{j}\right| \\
& Q(x, y)=a_{2}+b_{21} x+b_{22} y+\sum_{i=1}^{n} c_{2}^{i}\left|x-\gamma_{i}\right|+\sum_{j=1}^{m} d_{2}^{j}\left|y-\delta_{j}\right| .
\end{aligned}
$$

Como

$$
X_{\omega}(x, y)=\left(\begin{array}{cc}
\cos \omega & \operatorname{sen} \omega \\
-\operatorname{sen} \omega & \cos \omega
\end{array}\right)\left(\begin{array}{l}
P(x, y) \\
Q(x, y)
\end{array}\right),
$$

então o campo $X_{\omega}(x, y)=\left(P_{\omega}(x, y), Q_{\omega}(x, y)\right)$ pode ser escrito como:

$$
\begin{gathered}
P_{\omega}(x, y)=\left(a_{1} \cos \omega+a_{2} \operatorname{sen} \omega\right)+\left(b_{11} \cos \omega+b_{21} \operatorname{sen} \omega\right) x+\left(b_{12} \cos \omega+b_{22} \operatorname{sen} \omega\right) y+ \\
\sum_{i=1}^{n}\left(c_{1}^{i} \cos \omega+c_{2}^{i} \operatorname{sen} \omega\right)\left|x-\gamma_{i}\right|+\sum_{j=1}^{m}\left(d_{1}^{j} \cos \omega+d_{2}^{j} \operatorname{sen} \omega\right)\left|y-\delta_{j}\right|, \\
Q_{\omega}(x, y)=\left(-a_{1} \operatorname{sen} \omega+a_{2} \cos \omega\right)+\left(-b_{11} \operatorname{sen} \omega+b_{21} \cos \omega\right) x+\left(-b_{12} \operatorname{sen} \omega+b_{22} \cos \omega\right) y+ \\
\sum_{i=1}^{n}\left(-c_{1}^{i} \operatorname{sen} \omega+c_{2}^{i} \cos \omega\right)\left|x-\gamma_{i}\right|+\sum_{j=1}^{m}\left(-d_{1}^{j} \operatorname{sen} \omega+d_{2}^{j} \cos \omega\right)\left|y-\delta_{j}\right| .
\end{gathered}
$$

Logo, usando as equações encontradas em 2.19 e 2.20, e substituindo pelos termos equivalentes de $P_{\omega}$ e $Q_{\omega}$ encontramos a relação desejada.

\subsection{Tangências com a grade}

Introduziremos agora um conceito que será importante para a próxima seção.

Definição 2.2 Suponha que temos uma curva no plano definida por $f(p)=0$, onde $f$ é uma função de classe $C^{1}$ em que vale $\nabla f(p) \neq(0,0), \forall p \in f^{-1}(0)$. Tome $\varphi(t, p)$ o fluxo do campo de vetores $X$. Para $p_{0} \in f^{-1}(0)$, dizemos que o campo de vetores $X$ é tangente a curva em $p_{0}$ se

$$
\frac{\partial}{\partial t} f\left(\varphi\left(0, p_{0}\right)\right)=0
$$


ou seja

$$
\left\langle\nabla f\left(p_{0}\right), X\left(p_{0}\right)\right\rangle=0 .
$$

Além disso, se

$$
\frac{\partial^{2}}{\partial t^{2}} f\left(\varphi\left(0, p_{0}\right)\right) \neq 0
$$

dizemos que essa tangência é parabólica ou quadrática.

No caso que estamos estudando nos interessa como são as tangências de um campo $X$ com a grade $[\Gamma, \Delta]$.

Dessa forma podemos exprimir uma tangência vertical com a grade em pontos da forma $\left(\gamma_{i}, y\right)$ pela função $f(x, y)=x-\gamma_{i}$. Analogamente, tangências com a grade em pontos $\left(x, \delta_{j}\right)$ são expressos por $f(x, y)=y-\delta_{j}$.

Vamos terminar essa seção analisando o caso das tangências verticais. Dado um campo de vetores $X=(P, Q)$, considere a reta $x=\gamma_{i}$. Usando a definição anterior, temos uma tangência parabólica com essa reta se

$$
\begin{gathered}
\frac{\partial}{\partial t} f\left(\varphi\left(t, \gamma_{i}, y_{0}\right)\right)=\left\langle(1,0) ;\left(P\left(\gamma_{i}, y_{0}\right), Q\left(\gamma_{i}, y_{0}\right)\right)\right\rangle=P\left(\gamma_{i}, y_{0}\right)=0 \\
\frac{\partial^{2}}{\partial t^{2}} f\left(\varphi\left(t, \gamma_{i}, y_{0}\right)\right)=\left\langle\left(0, P_{y}\left(\gamma_{i}, y_{0}\right)\right) ;\left(0, Q\left(\gamma_{i}, y_{0}\right)\right)\right\rangle=P_{y}\left(\gamma_{i}, y_{0}\right) Q\left(\gamma_{i}, y_{0}\right) \neq 0 .
\end{gathered}
$$

Se eliminarmos o caso em que o ponto de tangência $\left(\gamma_{i}, y_{0}\right)$ é uma singularidade na grade $[\Gamma, \Delta]$, então temos $Q\left(\gamma_{i}, y_{0}\right) \neq 0$, e logo para a tangência ser parabólica deve valer $P_{y}\left(\gamma_{i}, y_{0}\right) \neq 0$.

Observe também que em cada intervalo $\Delta_{j}, j \in\{1,2, \ldots, m\}$, as tangências verticais são determinadas pela função linear $P\left(\gamma_{i}, y\right)=0$, logo se $P_{y}\left(\gamma_{i}, y\right) \neq 0$ então existe no máximo uma tangência do campo $X$ com a grade $[\Gamma, \Delta]$ em $\gamma_{i} \times \Delta_{j}$ e essa tangência é quadrática e isolada. 


\section{Capítulo 3}

\section{Estabilidade Estrutural}

Neste capítulo definiremos estabilidade estrutural em $\mathfrak{X}_{\Gamma, \Delta}$ e listaremos propriedades suficientes para que um campo de vetores $X$ seja estruturalmente estável. Tais condições são baseadas nos trabalhos de M. M. Peixoto e M. C. Peixoto [PP59] e J. Sotomayor [Sot85], onde temos uma rica análise do que acontece com tais condições quando os campos sofrem pequenas perturbações.

\subsection{Definição de Estabilidade Estrutural}

Em geral, para definirmos estabilidade estrutural, consideramos um espaço $\mathfrak{X}$ de campos de vetores em algum subconjunto $U \subset \mathbb{R}^{2}$ ou $\mathbb{S}^{2}$ e dotamos esse espaço com alguma topologia. Daí, dizemos que $X \in \mathfrak{X}$ é estruturalmente estável se existe uma vizinhança $\mathcal{U}$ de $X$ em $\mathfrak{X}$ tal que para todo $Y \in \mathcal{U}$ existe uma equivalência topológica definida em $U$, entre $X$ e $Y$.

Assim, como estamos considerando $\mathfrak{X}_{\Gamma, \Delta}$ com a topologia induzida pela métrica euclidiana de $\mathbb{R}^{N(\Gamma, \Delta)}$, e devido a relação desses campos com a grade $[\Gamma, \Delta]$, usando as notações introduzidas no capítulo anterior, é natural darmos a seguinte definição de estabilidade estrutural em $\mathfrak{X}_{\Gamma, \Delta}$.

Definição 3.1 Um elemento $X \in \mathfrak{X}_{\Gamma, \Delta}$ é dito estruturalmente estável se existe uma vizinhança $\mathcal{U}$ de $X$ em $\mathfrak{X}_{\Gamma, \Delta}$ tal que para todo $Y$ em $\mathcal{U}$ existe um homeomorfismo $h_{Y}$ : $\mathbb{D} \rightarrow \mathbb{D}$ que leva órbitas orientadas de $Y_{\mathbb{D}}$ em órbitas orientadas de $X_{\mathbb{D}}$ preservando $\partial \mathbb{D} e$ também a grade compactificada $[\Gamma, \Delta]$.

Com essas considerações, definimos $\mathscr{S}_{\Gamma, \Delta}$ como sendo o conjunto de todos os campos em $\mathfrak{X}_{\Gamma, \Delta}$ que são estruturalmente estáveis. 


\subsection{Propriedades de genericidade dos sistemas seccio- nalmente lineares}

Listaremos aqui algumas condições suficientes para que um campo de vetores $X \in \mathfrak{X}_{\Gamma, \Delta}$ esteja contido em $\mathscr{S}_{\Gamma, \Delta}$.

Tais condições estão no trabalho de J. Sotomayor e R. Garcia [SG03], e são reminiscentes das condições que aparecem no teorema de Andronov - Pontryagin - Peixoto. Usaremos a notação introduzida no capítulo anterior.

Definição 3.2 Um campo de vetores $X \in \mathfrak{X}_{\Gamma, \Delta}$ satisfaz as $[\Gamma, \Delta]$-condições para as singularidades se:

1. Todas as singularidades no infinito são hiperbólicas e disjuntas da direção dos eixos $x$ e $y$,

2. (respectivamente 2') Todas as suas singularidades finitas são hiperbólicas (respectivamente simples) e disjuntas das linhas $\Gamma \times \mathbb{R} e \mathbb{R} \times \Delta$,

3. Todas as tangências com as linhas $\Gamma \times \mathbb{R} e \mathbb{R} \times \Delta$ são quadráticas e disjuntas das esquinas $\Gamma \times \Delta$ da grade.

Definição 3.3 O conjunto dos campos de vetores satisfazendo as três condições será denotado por $\Sigma_{\Gamma, \Delta}(1)$, quando somente uma das condições for satisfeita (digamos a primeira), escreveremos $\Sigma_{\Gamma, \Delta}(1 ; 1)$, quando duas condições forem satisfeitas (digamos a primeira $e$ a terceira), escreveremos $\Sigma_{\Gamma, \Delta}(1 ; 1,3)$, e assim em diante.

Definição 3.4 Um campo de vetores $X \in \mathfrak{X}_{\Gamma, \Delta}$ satisfaz as $[\Gamma, \Delta]$-condições para órbitas periódicas se:

1. Todas as suas órbitas periódicas finitas são hiperbólicas e disjuntas das esquinas $\Gamma \times \Delta$ da grade e das tangências com as linhas $\Gamma \times \mathbb{R} e \mathbb{R} \times \Delta$,

2. A órbita periódica no infinito é hiperbólica.

Definição 3.5 O conjunto dos campos de vetores satisfazendo essas duas condições será denotado por $\Sigma_{\Gamma, \Delta}(2)$, quando somente uma dessas condições for satisfeita será escrito $\Sigma_{\Gamma, \Delta}(2 ; 1)$ ou $\Sigma_{\Gamma, \Delta}(2 ; 2)$, e assim por diante. 
Definição 3.6 Uma $[\Gamma, \Delta]$-conexão de separatrizes de $X$ é uma órbita finita de $X_{\mathbb{D}}$ que conecta:

1. dois pontos de sela que podem coincidir, nesse caso dizemos que temos uma conexão de selas ou, no caso de coincidência, um laço de sela,

2. um ponto de sela e um ponto de tangência, nesse caso dizemos que temos uma conexão de sela-tangência,

3. um ponto de sela e uma esquina da grade, nesse caso dizemos que temos uma conexão de sela-esquina,

4. dois pontos de tangência que podem coincidir, nesse caso dizemos que temos uma conexão de tangências ou, no caso de coincidência, um laço de tangência (o que é o mesmo que uma órbita periódica passando por uma tangência),

5. um ponto de tangência e uma esquina da grade, nesse caso dizemos que temos uma conexão de tangência-esquina,

6. dois pontos de esquina de grade que podem coincidir, nese caso dizemos que temos uma conexão de esquinas ou, no caso de coincidência, um laço de esquina (o que é o mesmo que uma órbita periódica passando por uma esquina).

Definição 3.7 O conjunto dos campos de vetores que não possuem $[\Gamma, \Delta]$-conexões de separatrizes será denotado por $\Sigma_{\Gamma, \Delta}(3)$.

Observação 3.1 Note que as conexões de sela contidas no infinito não foram consideradas conexões de separatrizes, isso ocorre pelo fato de que $\partial \mathbb{D}$ é invariante por $X_{\mathbb{D}}$, o que implica que essas conexões de sela persistem por pequenas perturbações.

\subsection{Teorema de Estabilidade e Genericidade}

Definidas essas condições e usando a notação da seção anterior podemos enunciar o seguinte teorema.

Teorema 3.1 Para o conjunto

$$
\Sigma_{\Gamma, \Delta}=\Sigma_{\Gamma, \Delta}(1) \cap \Sigma_{\Gamma, \Delta}(2) \cap \Sigma_{\Gamma, \Delta}(3)
$$

valem as seguintes afirmações: 


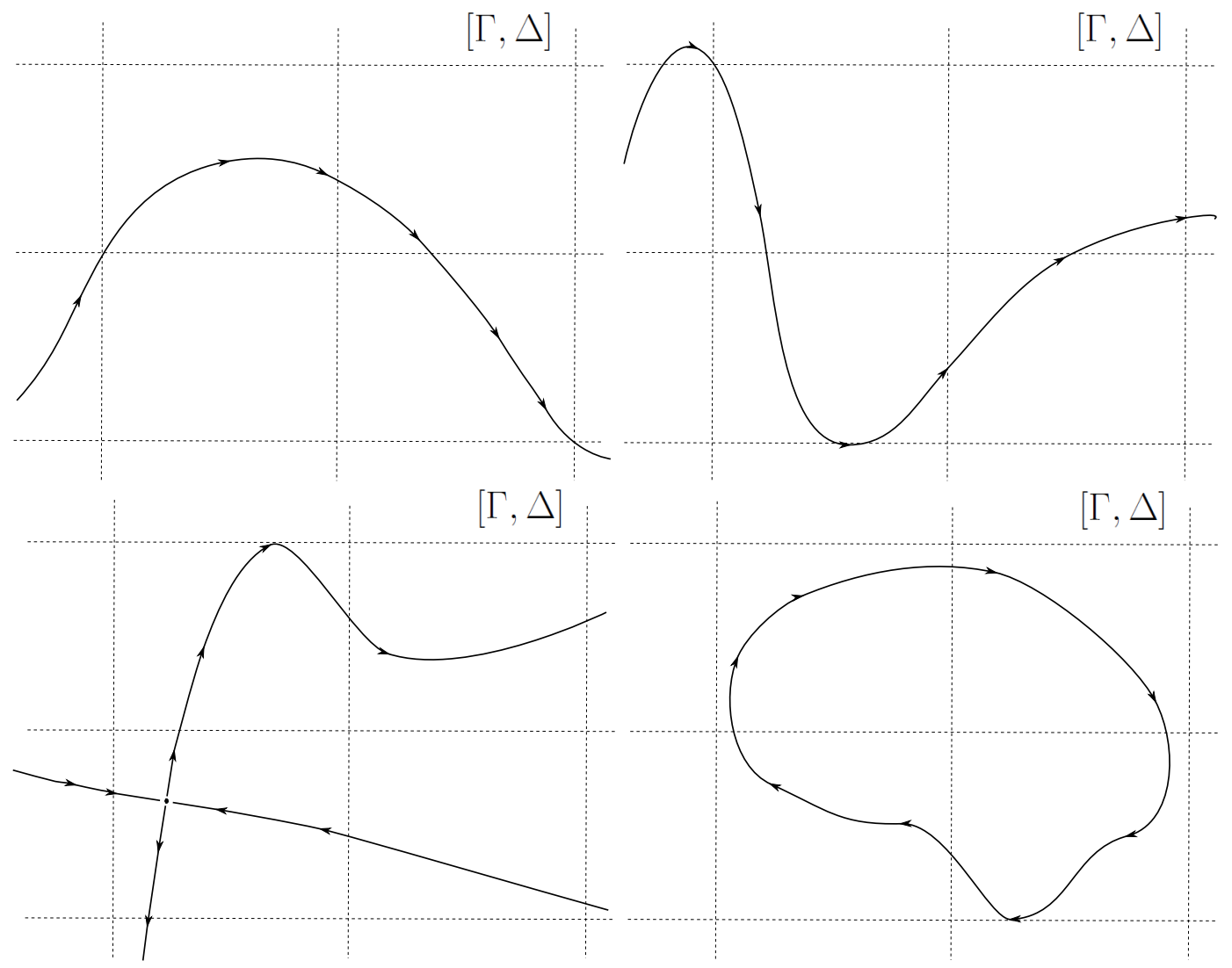

Figura 3.1: Exemplos de $[\Gamma, \Delta]$-conexões de separatrizes

1. $\Sigma_{\Gamma, \Delta}$ é aberto em $\mathfrak{X}_{\Gamma, \Delta}$,

2. $\Sigma_{\Gamma, \Delta}$ está contido no conjunto $\mathscr{S}_{\Gamma, \Delta}$,

3. $\Sigma_{\Gamma, \Delta}$ é denso em $\mathfrak{X}_{\Gamma, \Delta}$.

A demonstração da abertura no primeiro item e a construção do homeomorfismo no segundo item serão feitos pelo método das regiões canônicas com alguns ajustes necessários para garantir as condições relativas à grade $[\Gamma, \Delta]$.

A prova do terceiro item do teorema 3.1 será consequência do teorema 3.2, que garante que o complementar do conjunto $\Sigma_{\Gamma, \Delta}$, chamado de conjunto de bifurcação, tem medida de Lebesgue nula.

Teorema 3.2 O conjunto aberto $\Sigma_{\Gamma, \Delta}=\Sigma_{\Gamma, \Delta}(1) \cap \Sigma_{\Gamma, \Delta}(2) \cap \Sigma_{\Gamma, \Delta}(3)$ possui medida de Lebesgue total, em particular $\Sigma_{\Gamma, \Delta}$ é denso em $\mathfrak{X}_{\Gamma, \Delta}$.

Para provar o teorema anterior estudaremos o complementar do conjunto $\Sigma_{\Gamma, \Delta}$. 
Teorema 3.3 Para todo $X \in \mathfrak{X}_{\Gamma, \Delta}$ a família a cinco parâmetros definida por

$$
X_{\alpha_{1}, \alpha_{2}, \nu, \beta, \omega}(x, y)=R_{\omega}\left[X(x, y)+\alpha_{1} \frac{\partial}{\partial x}+\alpha_{2} \frac{\partial}{\partial y}+\nu\left(x \frac{\partial}{\partial x}+y \frac{\partial}{\partial y}\right)+\beta\left(-y \frac{\partial}{\partial x}+x \frac{\partial}{\partial y}\right)\right]
$$

intersecta o conjunto fechado $\mathfrak{X}_{\Gamma, \Delta}^{\prime}=\mathfrak{X}_{\Gamma, \Delta} \backslash \Sigma_{\Gamma, \Delta}$ em um conjunto de medida de Lebesgue nula.

A demonstração desses teoremas serão feitas no próximo capítulo.

\subsection{Lemas}

Apresentaremos agora alguns lemas que serão importantes para a demonstração da abertura do conjunto $\Sigma_{\Gamma, \Delta}$ e para a construção do homeomorfismo através do método das regiões canônicas.

Lema 3.1 Seja $X \in \Sigma_{\Gamma, \Delta}$ e seja $p_{E}$ um ponto singular finito hiperbólico de $X$. Então existem $\delta>0$ e uma vizinhança $\mathcal{V}_{p_{E}}$ de $p_{E}$ tal que essa vizinhança é um disco com bordo suave disjunto da grade $[\Gamma, \Delta]$. Todo $Y \in B_{\delta}(X)$ tem em $\mathcal{V}_{p_{E}}$ uma única singularidade $p_{E}(Y)$ do mesmo tipo que $p_{E}$ em $X$, isto é, uma sela, um nó ou um foco, atrator ou repulsor. Além disso, se $p_{E}$ não é uma sela, podemos tomar $\mathcal{V}_{p_{E}}$ tal que todo $Y \in B_{\delta}(X)$ é transversal a $\partial \mathcal{V}_{p_{E}}$.

Demonstração: Seja $X \in \Sigma_{\Gamma, \Delta}$ e tome $p_{E}$ um ponto singular finito de $X$. Como o campo está no conjunto $\Sigma_{\Gamma, \Delta}$, garantimos que existe uma célula $\Omega_{i j}$ tal que $p_{E}$ está no interior dessa célula.

Considere a função

$$
f: \mathbb{R}^{N(\Gamma, \Delta)} \times \mathbb{R}^{2} \rightarrow \mathbb{R}^{2}
$$

onde

$$
f(Y, q)=Y(q)
$$

Como o ponto $p_{E}$ é um ponto de equilíbrio de $X$, vale

$$
f\left(X, p_{E}\right)=X\left(p_{E}\right)=0 .
$$

Note também que, pelo fato de $p_{E}$ estar contido no interior de $\Omega_{i j}$, podemos, para todo $q \in \stackrel{\Omega}{\Omega}_{i j}$, escrever

$$
f(Y, q)=A_{i j}(Y) q+b_{i j}(Y)
$$


onde $A_{i j}(Y) \in \mathbb{M}_{2}(\mathbb{R})$ e $b_{i j}(Y) \in \mathbb{R}^{2}$ dependem continuamente de $Y$. Na verdade, com valores de $q$ restritos a célula $\Omega_{i j}, f$ é uma função analítica nas duas variáveis.

Logo, diferenciando $f$ com relação a $q$ e avaliando a diferencial no ponto $\left(X, p_{E}\right)$, temos

$$
D_{q} f\left(X, p_{E}\right)=A_{i j}(X)
$$

e como $p_{E}$ é um ponto singular hiperbólico de $X$, garantimos que $\operatorname{det} A_{i j}(X) \neq 0$.

Aplicando então o teorema da função implícita a $f$, temos que existe $\delta>0$, uma vizinhança $\mathcal{U}$ de $p_{E}$ e uma função $p_{E}: B_{\delta}(X) \rightarrow \mathcal{U}$ tal que

$$
f\left(Y, p_{E}(Y)\right)=Y\left(p_{E}(Y)\right)=0, \quad \forall Y \in B_{\delta}(X) .
$$

Diminuindo a vizinhança caso seja necessário, podemos considerar que $\mathcal{U}$ está contida no interior de $\Omega_{i j}$, e portanto é disjunta da grade $[\Gamma, \Delta]$.

Dessa forma provamos a existência e a unicidade do ponto singular $p_{E}(Y)$.

A hiperbolicidade e o tipo do ponto singular $p_{E}(Y)$ decorrem do fato de que as funções $\operatorname{det} A_{i j}(Y)$ e $\operatorname{tr} A_{i j}(Y)$ dependem continuamente de $Y$. Logo, diminuindo $\delta$ caso seja necessário, garantimos que $p_{E}(Y)$ é hiperbólico e do mesmo tipo que $p_{E}$, isto é, uma sela, um nó ou um foco. Além disso, se $p_{E}$ não for uma sela, $p_{E}(Y)$ tem o mesmo critério de estabilidade de $p_{E}$, ou seja, é um ponto atrator ou repulsor.

Suponha agora que $p_{E}$ não é uma sela. Vamos escolher $\mathcal{V}_{p_{E}}$ de tal forma que todo campo em $B_{\delta}(X)$ seja transversal ao bordo dessa vizinhança. Como enunciado em [Sot79,p.74], pelo fato do campo $X$ ser linear em $\Omega_{i j}$, existe uma forma quadrática, a saber

$$
\mathcal{Q}(q)=\int_{0}^{\infty}\left\langle e^{t A_{i j}} q, e^{t A_{i j}} q\right\rangle d t
$$

que é uma função de Lyapunov estrita para o campo $X$ em uma vizinhança de $p_{E}$. Como essa função é contínua e $\mathcal{Q}\left(p_{E}\right)=0$, podemos então escolher um dos níveis dessa forma quadrática como $\partial \mathcal{V}_{p_{E}} \operatorname{com} \mathcal{V}_{p_{E}} \subset \mathcal{U}$.

A transversalidade de $Y$ a $\partial \mathcal{V}_{p_{E}}$ resultará da estabilidade assintótica do ponto singular $p_{E}$ de $X($ ou $-X)$. De fato, como $p_{E}$ é assintoticamente estável e $\mathcal{Q}$ é uma função de Lyapunov estrita para $p_{E}$, então para todo $q \in \partial \mathcal{V}_{p_{E}}$, vale

$$
\langle\nabla \mathcal{Q}(q), X(q)\rangle<0
$$

Considere a função $\mathcal{G}(q, Y)=\langle\nabla \mathcal{Q}(q), Y(q)\rangle$. Essa função é contínua nas duas variáveis. Por $\partial \mathcal{V}_{p_{E}}$ ser compacto, tomemos

$$
\max \left\{\mathcal{G}(q, X) \mid q \in \partial \mathcal{V}_{p_{E}}\right\}=M<0
$$

Diminuindo $\delta$ caso seja necessário, para todo $Y \in B_{\delta}(X), \mathcal{G}(q, Y)<0, \forall q \in \partial \mathcal{V}_{p_{E}}$, ou 
seja, todo $Y$ em $B_{\delta}(X)$ é transversal ao bordo de $\mathcal{V}_{p_{E}}$.

De fato, se isso não ocorresse, existiriam sequências $\left(Y_{n}\right)_{n \in \mathbb{N}}$ de campos em $\mathfrak{X}_{\Gamma, \Delta}$ e $\left(q_{n}\right)_{n \in \mathbb{N}}$ de pontos em $\partial \mathcal{V}_{p_{E}}$ tal que

$$
Y_{n} \underset{n \rightarrow \infty}{\longrightarrow} X
$$

e

$$
q_{n} \underset{n \rightarrow \infty}{\longrightarrow} \tilde{p}
$$

$\operatorname{com} \tilde{p} \in \partial \mathcal{V}_{p_{E}}$, onde

$$
\mathcal{G}\left(q_{n}, Y_{n}\right) \geqslant 0
$$

Mas, como $\mathcal{G}$ é contínua e vale 3.1, temos

$$
\mathcal{G}\left(q_{n}, Y_{n}\right) \underset{n \rightarrow \infty}{\longrightarrow} \mathcal{G}(\tilde{p}, X) \leqslant M<0
$$

o que é uma contradição.

Isso termina a demonstração do lema.

Os campos em $\Sigma_{\Gamma, \Delta}$ possuem no máximo $(n+1)(m+1)$ singularidades finitas. Logo, se $p_{E_{1}}, p_{E_{2}}, \ldots, p_{E_{k}}$ são os pontos singulares finitos de $X$, podemos escolher $\delta>0$ e vizinhanças $\mathcal{V}_{p_{E_{s}}}$ de $p_{E_{s}}$ tal que o enunciado do lema anterior seja válido para todo campo $Y \in B_{\delta}(X)$ e todo ponto singular finito $p_{E_{s}}$.

Lema 3.2 Seja $X \in \Sigma_{\Gamma, \Delta}$ e seja $\gamma$ uma órbita periódica finita de $X$. Então existem $\delta>0$ e uma vizinhança $\mathcal{V}_{\gamma}$ de $\gamma$ tal que $\mathcal{V}_{\gamma}$ é uma coroa com bordo suave disjunta das esquinas da grade $[\Gamma, \Delta]$ e transversal a $Y$ para todo $Y \in B_{\delta}(X)$. Cada $Y \in B_{\delta}(X)$ tem em $\mathcal{V}_{\gamma}$ uma única órbita periódica $\gamma(Y)$ do mesmo tipo que $\gamma$ de $X$, isto é, hiperbólica atratora ou repulsora de acordo com o caso de $\gamma$. Além disso todas as órbitas de $Y$ em $\mathcal{V}_{\gamma}$ são transversais à grade $[\Gamma, \Delta]$.

Demonstração: Considere $X \in \Sigma_{\Gamma, \Delta}$ e seja $\gamma$ uma órbita periódica finita de $X$. Como o campo está no conjunto $\Sigma_{\Gamma, \Delta}$, garantimos que a órbita periódica $\gamma$ é hiperbólica e não possui pontos de esquina nem pontos de tangência com a grade $[\Gamma, \Delta]$.

Seja

$$
d=d(\gamma, \Gamma \times \Delta)
$$

a distância entre o conjunto dos pontos de esquina da grade $\Gamma \times \Delta$ e a órbita $\gamma$. Pelas considerações anteriores concluímos que $d>0$. 
Tome

$$
V_{\epsilon}=\left\{p \in \mathbb{R}^{2} \mid d(p, \gamma)<\epsilon\right\},
$$

onde $0<\epsilon<d$. Se $\epsilon$ for pequeno o suficiente, $V_{\epsilon}$ é uma vizinhança de $\gamma$ que não possui outras órbitas periódicas de $X$ nem pontos de esquina da grade.

Considere agora a família de campos rotacionados dada por

$$
X_{\omega}=R_{\omega}(X)
$$

Aplicando os resultados de [Duf53] sobre famílias de campos vetoriais rotacionados, concluímos que, por $\gamma$ ser hiperbólica existem, $C_{1}$ e $C_{2}$ órbitas periódicas dos campos $R_{\omega_{1}}(X)$ e $R_{\omega_{2}}(X)$ obtidas rotacionando o campo de vetores $X$ em sentidos opostos, ou seja, $\omega_{1}<0<\omega_{2}$, uma dessa órbitas está contida no interior de $\gamma$ e a outra contém $\gamma$, e $C_{1}$ e $C_{2}$ estão contidos em $V_{\epsilon}$.

Observemos ainda que o campo $X$ é transversal às curvas $C_{1}$ e $C_{2}$. De fato, os campos $X$ e $X_{\omega_{1}}$ formam um ângulo constante $\omega_{1}$ sobre a curva $C_{1}$ e $X$ e $X_{\omega_{2}}$ formam um ângulo constante $\omega_{2}$ sobre a curva $C_{2}$.

De forma análoga a demonstração no lema anterior, podemos encontrar um $\delta>0$ tal que todo $Y$ em $B_{\delta}(X)$ seja transversal as curvas $C_{1}$ e $C_{2}$.

Tome como vizinhança $\mathcal{V}_{\gamma}$ o anel cujo bordo é formado pelas curvas $C_{1}$ e $C_{2}$.

Vamos mostrar que, diminuindo $\delta$ caso seja necessário, todo $Y \in B_{\delta}(X)$ tem em $\mathcal{V}_{\gamma}$ uma única órbita periódica hiperbólica do mesmo tipo que $\gamma$.

Seja $L_{0}$ uma seção transversal para o $\gamma$ em $p_{0}$. Podemos considerar que $L_{0}$ está inteiramente contido em uma célula da grade $[\Gamma, \Delta]$.

Pelas observações 2.3 e 2.8 , se definirmos o campo de vetores $\Lambda: \mathbb{R}^{2} \times \mathbb{R}^{N(\Gamma, \Delta)} \rightarrow$ $\mathbb{R}^{2} \times \mathbb{R}^{N(\Gamma, \Delta)}$ por

$$
\Lambda(p, Y)=(Y(p), 0)
$$

diminuindo $\delta$ caso seja necessário, encontramos uma aplicação de retorno $\Pi$ para um ponto $\left(p_{0}, X\right)$ da órbita periódica $\Phi(t)=(\gamma(t), X)$, com $\Pi$ definida na seção transversal $\Sigma_{0}=L_{0} \times B_{\delta}(X)$.

Facilmente vemos que essa aplicação de retorno tem a seguinte forma

$$
\Pi(s, Y)=(\pi(s, Y), Y),
$$

supondo que $L_{0}$ é parametrizado por $\sigma: I \rightarrow \mathbb{R}^{2}, 0 \in I$ e $\sigma(0)=p_{0}$. Sendo que $\pi(s, X)$ é a aplicação de retorno de $\gamma$ em $L_{0}$.

Como $\gamma$ é uma órbita periódica hiperbólica

$$
\frac{\partial}{\partial s} \pi(0, X) \neq 1
$$


Aplicando o teorema da função implícita para

$$
f(s, X)=\pi(s, X)-s
$$

encontramos, que diminuindo $\delta$ caso seja necessário, existem $J$ vizinhança de 0 e uma função $s: B_{\delta}(X) \rightarrow J$ tal que $\delta(s(Y))$ é um ponto em $L_{0}$ que pertence a uma órbita periódica de $Y$ inteiramente contida em $\mathcal{V}_{\gamma}$, sendo essa órbita periódica a única de $Y$ em $\mathcal{V}_{\gamma}$.

Chame de $\gamma(Y)$ a órbita de $Y$ em $\mathcal{V}_{\gamma}$. Falta mostrarmos que para todo $Y$ essas órbitas são hiperbólicas e possuem o mesmo caráter de estabilidade que $\gamma$.

Para isso vamos encontrar uma fórmula para a derivada da aplicação de retorno para cada uma dessas órbitas.

Sabemos que a órbita periódica $\gamma$ está contida em um número finito de células da grade $[\Gamma, \Delta]$. Sejam então $\left\{\Omega_{i_{0} j_{0}}, \Omega_{i_{1} j_{1}}, \ldots, \Omega_{i_{k} j_{k}}\right\}$ as células da grade que contém pontos da órbita $\gamma$.

Como os campos em $\mathfrak{X}_{\Gamma, \Delta}$ são lineares em cada célula $\Omega_{i j}$, dado $X$, temos que $\operatorname{div} X(\varphi(t))$ é igual a $\operatorname{tr} A_{i j}(X)$, enquanto a curva $\varphi(t)$ permanecer em $\Omega_{i j}$.

Pela proposição 2.5, sabemos que a derivada da aplicação de retorno é

$$
\pi^{\prime}(X, 0)=\exp \left(\sum_{r=0}^{k} \operatorname{tr} A_{i_{r} j_{r}}(X)\left(t_{r+1}-t_{r}\right)\right) \neq 1
$$

onde a expressão de $\operatorname{tr} A_{i j}(Y)$ é

$$
\operatorname{tr} A_{i j}(Y)=b_{11}+b_{22}+\sum_{i^{\prime}=1}^{i} c_{1}^{i^{\prime}}-\sum_{i^{\prime}=i+1}^{n} c_{1}^{i^{\prime}}+\sum_{j^{\prime}=1}^{j} d_{2}^{j^{\prime}}-\sum_{j^{\prime}=j+1}^{m} d_{2}^{j^{\prime}}
$$

Considerando as aplicações de transição entre a seção transversal e os lados da grade $[\Gamma, \Delta]$, pelo teorema 2.1, concluímos que existem funções contínuas $\tilde{\tau}_{r}, r=1,2, \ldots, k$, que determinam o tempo mínimo necessário para que a órbita, ao passar por um ponto da grade, intersecte a grade novamente.

Todas essas funções são contínuas, logo fazendo a composição $\tilde{\tau}_{r} \operatorname{com}$ a função $s(Y)$ encontramos o tempo $\tau_{r}(Y)$ que um ponto da órbita periódica $\gamma(Y)$ leva para ir de um lado da grade para outro, assim, podemos escrever a derivada da transformação de retorno para $\gamma(Y)$ como

$$
\pi^{\prime}(Y, s(Y))=\exp \left(\sum_{r=0}^{k} \operatorname{tr} A_{i_{r} j_{r}}(Y)\left(\tau_{r+1}(Y)-\tau_{r}(Y)\right)\right) .
$$

Observe que essa função é contínua, pois $\operatorname{tr} A_{i j}(Y)$ e $\tau_{r}(Y)$ são contínuas, logo diminuindo $\delta$ caso seja necessário, para todo $Y \in B_{\delta}(X)$, temos que $\pi^{\prime}(Y, s(Y)) \neq 1$, e 
portanto $\gamma_{k}(Y)$ é hiperbólica e tem o mesmo caráter de estabilidade que $\gamma$.

Isso termina a demonstração do lema.

Observe que os campos em $\Sigma_{\Gamma, \Delta}$ possuem um número finito de órbitas periódicas finitas. Logo, se $\gamma_{1}, \gamma_{2}, \ldots, \gamma_{j}$ são as órbitas periódicas finitas de $X$, podemos tomar $\delta>0$ e vizinhanças $\mathcal{V}_{\gamma_{s}}$ de $\gamma_{s}$ tal que vale o enunciado do lema anterior para todo $Y \in B_{\delta}(X)$ e toda órbita periódica finita $\gamma_{s}$.

Lema 3.3 Seja $X \in \Sigma_{\Gamma, \Delta}$ e seja $p_{I}$ uma singularidade hiperbólica infinita de $X$. Então existe $\delta>0$ e uma vizinhança $\mathcal{V}_{p_{I}}$ de $p_{I}$ tal que $\mathcal{V}_{p_{I}}$ é um disco com bordo suave disjunto da grade $[\Gamma, \Delta]$. Todo $Y \in B_{\delta}(X)$ tem em $\mathcal{V}_{p_{I}}$ uma única singularidade $p_{I}(Y)$, sendo ela infinita, do mesmo tipo que $p_{I}$ em $X$. Além disso se $p_{I}$ não é uma sela podemos tomar $\mathcal{V}_{p_{I}}$ tal que todo $Y \in B_{\delta}(X)$ é transversal ao bordo de $\mathcal{V}_{p_{I}}$

Demonstração: A demonstração desse lema é semelhante a demonstração do lema 3.1. De fato se $p_{I}=\left(\theta_{I}, 0\right)$ é um ponto singular infinito de $X$ basta analisar o campo nas coordenadas $(\theta, \rho)$ e observar que a equação para as singularidades hiperbólicas no infinito são dadas pelas funções

$$
A_{1}(Y)(\theta, 0)=0
$$

e

$$
\left(\frac{\partial}{\partial \theta} A_{1}(Y)(\theta, 0)\right) R_{1}(Y)(\theta, 0) \neq 0 .
$$

Como em pontos próximos de $p_{I}$ essas funções são diferenciáveis em $\theta$ e $Y$, garantimos a existência, a unicidade e o tipo do ponto hiperbólico $p_{I}(Y)$.

Se $p_{I}$ não for uma sela basta usarmos a forma quadrática

$$
Q(p)=\int_{0}^{\infty}\left\langle e^{M t} p, e^{M t} p\right\rangle d t
$$

onde $M=D X\left(\theta_{i}, 0\right)$ para garantir que $Y$ é transversal ao bordo de $\mathcal{V}_{p_{I}}$.

Pela proposição 2.7, concluímos que um campo $X$ em $\Sigma_{\Gamma, \Delta}$ possui no máximo 8 singularidades no infinito, podendo ser 2 em cada quadrante. Logo, se $p_{I_{1}}, \ldots, p_{I_{k}}$ são as singularidade no infinito de $X$, podemos tomar $\delta>0$ tal que o lema anterior seja válido para todo $Y \in B_{\delta}(X)$ e toda singularidade infinita $p_{I_{s}}$.

Lema 3.4 Seja $X \in \Sigma_{\Gamma, \Delta}$ e seja $\psi$ a órbita periódica no infinito de $X$. Então existe 


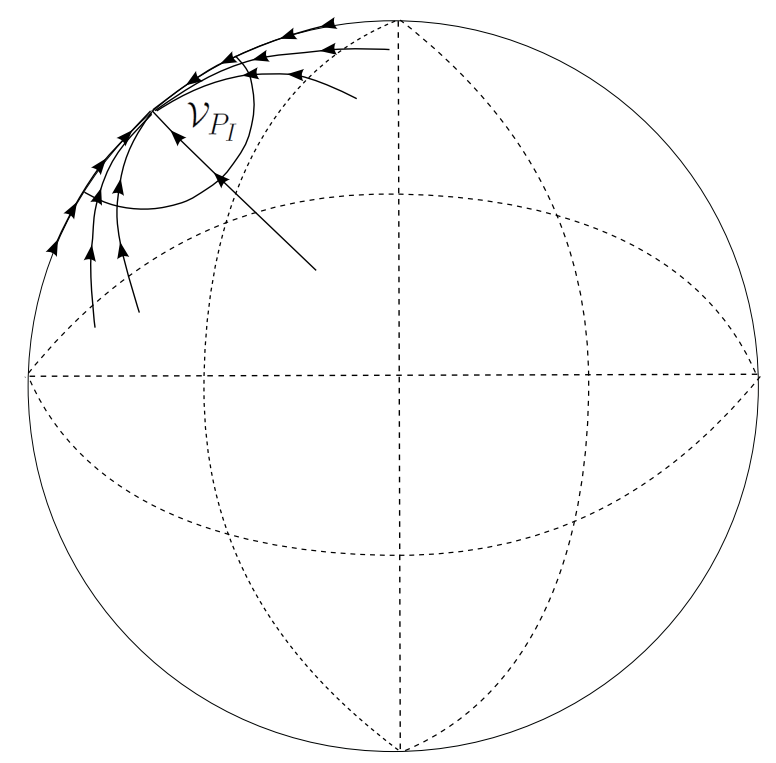

Figura 3.2: Vizinhança $\mathcal{V}_{p_{I}}$ da singularidade no infinito

$\delta>0$ e uma vizinhança $\mathcal{V}_{\psi}$ de $\psi$ tal que todo $Y \in B_{\delta}(X)$ é transversal ao bordo de $\mathcal{V}_{\psi}$ e tem a órbita no infinito $\psi_{Y}$ como única órbita periódica em $\mathcal{V}_{\psi}$ mantendo o seu caráter de estabilidade.

Demonstração: Seja $X \in \mathfrak{X}_{\Gamma, \Delta}$ e suponha que o infinito é uma órbita periódica hiperbólica para o campo. Observe que para termos uma órbita periódica no infinito devemos ter $A_{1}(X)(\theta, 0) \neq 0, \forall \theta \in[0,2 \pi]$. Como a função $A_{1}(Y)(\theta, 0)$ é contínua em $Y$ e $\theta$, e $[0,2 \pi]$ é compacto, garantimos que existe $\delta>0$ tal que para todo $Y \in B_{\delta}(X)$ vale $A_{1}(Y)(\theta, 0) \neq 0$, $\forall \theta \in[0,2 \pi]$.

Logo $\psi_{Y}$ é uma órbita periódica para $Y$. A estabilidade de $\psi_{Y}$ decorre do fato de que

$$
\pi_{Y}^{\prime}(0)=\exp \left[\sum_{j=1}^{4} \int_{\theta_{j-1}}^{\theta_{j}}-\frac{R_{1 j}(Y)(\theta)}{A_{1 j}(Y)(\theta)} d \theta\right]
$$

é uma função contínua para $Y \in B_{\delta}(X)$, logo, diminuindo $\delta$ caso seja necessário, temos $\pi_{Y}^{\prime}(0) \neq 1$. Assim garantimos o critério de estabilidade de $\psi_{Y}$.

Para encontrarmos a vizinhança $\mathcal{V}_{\psi}$, basta considerarmos o campo $X_{\mathbb{D}}$. Assim, se tomarmos um campo rotacionado $R_{\omega}\left(X_{\mathbb{D}}\right)$, pela teoria de campos rotacionados [Duf53], existe um $\omega$ próximo de zero, e uma curva $C$ contida no interior de $\partial \mathbb{D}$, tal que $C$ é uma órbita periódica de $R_{\omega}\left(X_{\mathbb{D}}\right)$, e $X_{\mathbb{D}}$ é transversal a $C$. Logo, analogamente ao que fizemos nos lemas anteriores, existe um $\delta>0$ tal que todo $Y \in B_{\delta}(X)$ é transversal a $C$. Assim, podemos tomar como vizinhança de $\psi$ a região que está contida no exterior de $C$ e no interior $\mathbb{D}$. Como queríamos. 


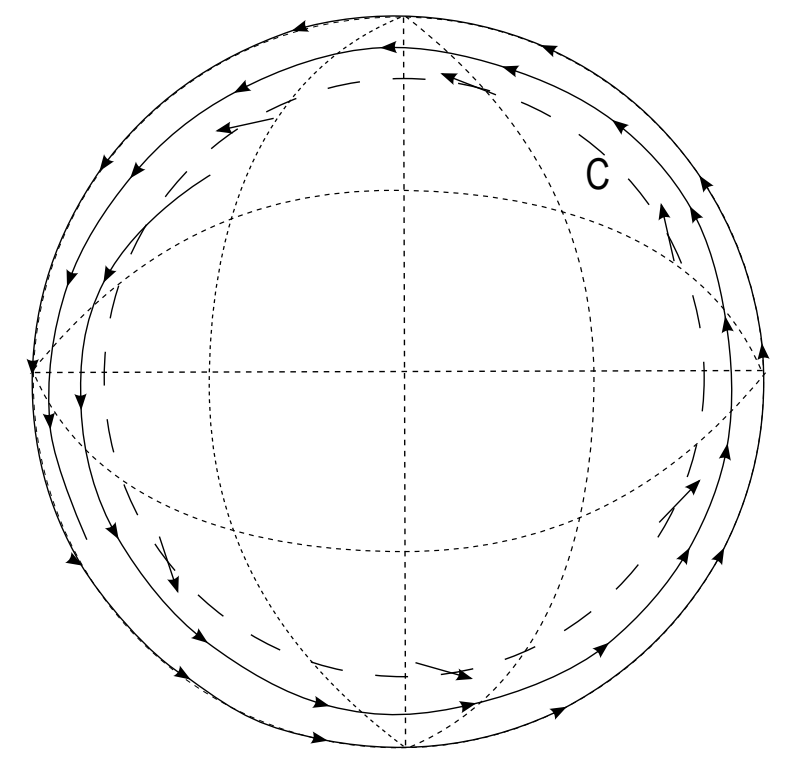

Figura 3.3: Vizinhança da órbita periódica no infinito

Lema 3.5 Seja $X \in \Sigma_{\Gamma, \Delta}$. Se $\delta>0$ é suficientemente pequeno e $Y \in B_{\delta}(X)$, então todo ponto singular de $Y$ está no interior das vizinhanças dadas por 3.1 e 3.3 e toda órbita periódica de $Y$ está no interior das vizinhanças dadas por 3.2 e 3.4.

Demonstração: A primeira afirmação do lema é facilmente verificada levando em consideração a continuidade do fluxo com relação aos campos e condições iniciais.

Para a segunda afirmação considere $\mathrm{C}$ como sendo o fecho do conjunto

$$
\mathbb{D}-\left[\mathcal{V}_{\psi} \cup\left(\bigcup_{j=1}^{k} \mathcal{V}_{\gamma_{j}}\right)\right]
$$

onde $\psi$ é a órbita periódica no infinito, $\gamma_{j}, j=1,2, \ldots, k$ as órbitas periódicas finitas de $X$ e $\mathcal{V}_{\gamma_{j}}$ e $\mathcal{V}_{\psi}$ são as vizinhanças dadas pelos lemas 3.2 e 3.4 .

Em virtude dos lemas 3.2 e 3.4 nós temos apenas que mostrar que existe $\delta>0$ tal que qualquer trajetória de $Y \in B_{\delta}(X)$ passando por um ponto de $C$ não é fechada.

Primeiro considere $C^{\prime} \subset C$ o conjunto obtido de $C$ retirando o interior de todas as vizinhanças $\mathcal{V}_{p_{S}}$ contendo pontos de sela de $X$. Esse conjunto é fechado em $\mathbb{D}$ e portanto compacto.

Afirmamos que para $\delta$ suficientemente pequeno, $Y \in B_{\delta}(X)$ não possui órbitas periódicas em $C^{\prime}$. De fato, se isso não ocorresse existiriam sequências $\left(Y_{n}\right)_{n \in \mathbb{N}}$ e $\left(p_{n}\right)_{n \in \mathbb{N}}$ tais que

$$
Y_{n} \underset{n \rightarrow \infty}{\longrightarrow} X
$$

e

$$
p_{n} \underset{n \rightarrow \infty}{\longrightarrow} \tilde{p}
$$


com $\tilde{p} \in C^{\prime}$, tal que $\varphi\left(t, p_{n}, Y_{n}\right)$ é uma órbita periódica contida em $C^{\prime}$.

Seja $\varphi(t, \tilde{p}, X)$ a trajetória de $X$ passando por $\tilde{p}$. Sabemos que $\varphi(t, \tilde{p}, X)$ não é fechada, dado que estamos assumindo que todas as órbitas periódicas de $X$ estão contidas em $\mathcal{V}_{\psi} \cup\left(\bigcup_{j=1}^{k} \mathcal{V}_{\gamma_{j}}\right)$

Então $\tilde{p}$ divide a trajetória em duas partes, e como estamos supondo que $X \in \Sigma_{\Gamma, \Delta}$, pelo menos uma dessas semi-órbitas não tende para um ponto de sela. Essa semi-órbita tende para um ciclo limite, um foco ou um nó. Pela continuidade do fluxo com relação aos campos e condições iniciais, para $p_{n}$ próximo de $\tilde{p}$ a órbita $\varphi\left(t, p_{n}, Y_{n}\right)$ entrará numa vizinhança $\mathcal{V}_{\lambda}$, onde $\lambda$ é um foco, nó ou ciclo limite, e permanecerá nessa vizinhança, pois o bordo da região é transversal a $Y$. Como a outra semi-órbita não pode entrar na mesma vizinhança, concluímos que essa trajetória não é fechada. Isso gera uma contradição.

Então existe $\delta>0$ tal que toda trajetória de $Y \in B_{\delta}(X)$ através de pontos de $C^{\prime}$ não é fechada.

Vemos facilmente também que qualquer trajetória de $Y$ através de um ponto de $C \backslash C^{\prime}$ não é fechada. De fato seja $\varphi(t, q, Y)$ uma trajetória de $Y$ através de $q \in \mathcal{V}_{p_{S}}$, onde $\mathcal{V}_{p_{S}}$ contém um ponto de sela. Se essa trajetória fosse fechada deveria estar contida em $\mathcal{V}_{p_{S}}$, mas nessa vizinhança o campo é linear não homogêneo com sua única singularidade sendo um ponto de sela, logo não existem órbitas periódicas em $\mathcal{V}_{p_{S}}$.

Isso termina a demonstração do lema.

Observação 3.2 Podemos escolher as vizinhanças dadas pelos lemas 3.1 e 3.3 pequenas o suficiente tal que se $p_{S}$ é um ponto de sela de $X$ então nenhuma outra separatriz de um outro ponto de sela de $X$ intersecta a vizinhança de $\mathcal{V}_{p_{S}}$.

Observação 3.3 Seja agora $p_{T_{i}}, i=1,2,3, \ldots, k$ os pontos de tangência de $X$ com a grade compactificada $[\Gamma, \Delta]$. Para todo $p_{T_{i}}$, escolhemos um pequeno arco aberto $\lambda_{i}$ de $[\Gamma, \Delta]$ contendo $p_{T_{i}}$ tal que todos esses arcos são disjuntos e não contém nenhum ponto relativo às outras separatrizes do sistema $X$. Isso é possível pois estamos considerando $X \in \Sigma_{\Gamma, \Delta}$. Seja $K$ o fecho da grade compactificada em $\mathbb{D}$.

Considere agora o conjunto compacto

$$
L=K-\bigcup_{i=1}^{n} \lambda_{i}
$$

Pela forma como esse conjunto é construído, garantimos que $X$ é transversal a $L$ em todos os pontos.

Pela continuidade do fluxo com relação aos parâmetros e condições iniciais e por $L$ ser compacto, podemos então escolher $\delta>0$ tal que todo $Y \in B_{\delta}(X)$ é transversal ao conjunto $L$. 
De agora em diante sempre que tomarmos $\delta>0$, estaremos considerando que esse valor é tal que sejam válidos os lemas 3.1, 3.2, 3.3, 3.4 e 3.5, bem como a observação anterior.

Definição 3.8 Uma fonte (respectivamente um poço) de $X_{\mathbb{D}}$ em $\mathbb{D}$ é um nó, um foco ou uma órbita periódica, tal que órbitas próximas tendem para esse conjunto quando $t \rightarrow-\infty$ (respectivamente $t \rightarrow \infty$ ).

Analisaremos a seguir o comportamento das órbitas de $X$ que passam por pontos de tangência, pontos de esquina ou são separatrizes de sela do sistema.

É de fundamental importância entendermos como tais órbitas se comportam quando consideramos campos em $\mathfrak{X}_{\Gamma, \Delta}$ próximos de $X$, pois como veremos mais adiante, essas trajetórias são importantes para a determinação das regiões canônicas e a construção do homeomorfismo que conjuga os campos.

Observação 3.4 Considere $X$ um campo em $\Sigma_{\Gamma, \Delta}$ e $\varphi$ uma órbita que liga uma fonte $\alpha$ com um poço $\omega$, sendo neste caso esses últimos formados por pontos singulares hiperbólicos ou órbitas periódicas hiperbólicas do campo. Considere $\mathcal{V}_{\alpha}$ e $\mathcal{V}_{\omega}$ respectivamente as vizinhanças da fonte e do poço dados pelos lemas 3.1, 3.2, 3.3 e 3.4.

O conjunto $[\Gamma, \Delta] \cup \partial \mathcal{V}_{\alpha} \cup \partial \mathcal{V}_{\omega}$ formado pelos pontos da grade $[\Gamma, \Delta]$ que não estão no interior de $\mathcal{V}_{\alpha} \cup \mathcal{V}_{\omega}$ particiona a órbita $\varphi$ em um número finito de arcos. De fato, se percorrermos a órbita $\varphi$ no sentido positivo, vemos facilmente que existe uma sequência finita de pontos $E_{1}, E_{2}, \ldots, E_{n}, \operatorname{com} E_{1} \in \partial \mathcal{V}_{\alpha}, E_{n} \in \partial \mathcal{V}_{\omega}$ e $E_{j} \in[\Gamma, \Delta], j=2,3, \ldots, n-1$, de forma que os arcos mencionados são os arcos de trajetória com extremos em $E_{j}$ e $E_{j+1}$.

No máximo um ponto $E_{j}$ pode ser um ponto de tangência ou esquina da grade. Em todos os outros pontos $\varphi$ intersecta o conjunto $[\Gamma, \Delta] \cup \partial \mathcal{V}_{\alpha} \cup \partial \mathcal{V}_{\omega}$ transversalmente.

Na figura 3.4 temos as órbitas $\varphi$ e $\tilde{\varphi}$ ilustrando essa situação.

Observação 3.5 O mesmo vale para órbitas que são separatrizes de sela, se considerarmos que, nesse caso, para uma separatriz instável $p_{S}=E_{1}$ e para uma separatriz estável $p_{S}=E_{n}$, onde $p_{S}$ é o ponto de sela.

Lema 3.6 Seja $X \in \Sigma_{\Gamma, \Delta}, p_{T}$ um ponto de tangência com a grade $[\Gamma, \Delta]$ e $\lambda$ o arco contendo $p_{T}$ escolhido como na observação 3.3. Então existe $\delta>0$ tal que todo $Y \in B_{\delta}(X)$ satisfaz as seguintes propriedades:

(a) - Y tem em $\lambda$ um único ponto de tangência parabólica $p_{T}(Y)$ com a grade e do mesmo tipo que $p_{T}$, isto é, externa ou interna com relação a alguma célula de $[\Gamma, \Delta]$; 


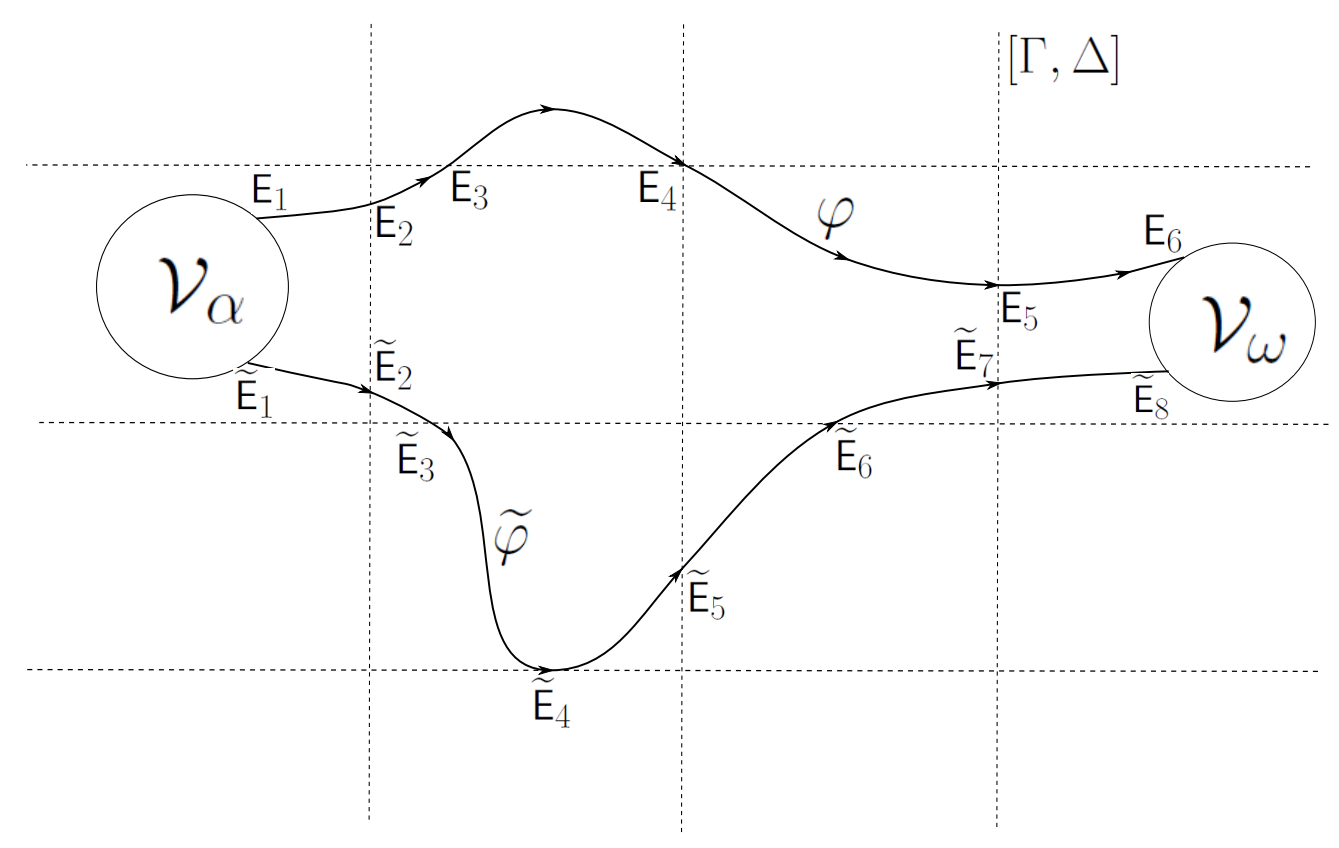

Figura 3.4: Casos com tangência e pontos de esquina

(b) - Se $\varphi\left(t, p_{T}, X\right)$ liga uma fonte $\alpha_{X}$ com um poço $\omega_{X}$, então $\varphi\left(t, p_{T}(Y), Y\right)$ liga uma fonte $\alpha_{Y}$ e um poço $\omega_{Y}$ contidos respectivamente nas vizinhanças $\mathcal{V}_{\alpha_{X}}$ e $\mathcal{V}_{\omega_{X}}$;

(c) - A órbita $\varphi\left(t, p_{T}(Y), Y\right)$ não contém pontos de esquina de $[\Gamma, \Delta]$ e contém somente $p_{T}(Y)$ como ponto de tangência;

(d) - Se $E_{j}, j=1,2, \ldots, n$, são os pontos descritos na observação 3.4, com $E_{j_{0}}=p_{T}$, então $\varphi\left(t, p_{T}(Y), Y\right)$ intersecta o conjunto $[\Gamma, \Delta] \cup \partial \mathcal{V}_{\alpha_{X}} \cup \partial \mathcal{V}_{\omega_{X}}$ em pontos $E_{j}(Y), j=$ $1,2, \ldots, n$, com $E_{j}(Y)$ próximo de $E_{j}$, e com exceção de $E_{j_{0}}(Y)=p_{T}(Y)$, sempre o faz transversalmente e disjunto das esquinas da grade.

Demonstração: Seja $p_{T}$ um ponto de tangência de $X$ com a grade $[\Gamma, \Delta]$. Sem perda de generalidade podemos supor $p_{T}=\left(x_{T}, \delta_{j}\right)$ uma tangência horizontal com a reta $y=\delta_{j}$.

Como $p_{T}$ é uma tangência parabólica, temos

$$
Q\left(x_{T}, \delta_{j}, X\right)=0
$$

e

$$
Q_{x}\left(x_{T}, \delta_{j}, X\right) \neq 0
$$

Se $Q_{x}\left(x_{T}, \delta_{j}, X\right)>0$ (respectivamente $\left.Q_{x}\left(x_{T}, \delta_{j}, X\right)<0\right)$, então para valores pequenos de $t, \varphi\left(t, p_{T}, X\right)$ está contida na célula abaixo (respectivamente acima) da reta $y=\delta_{j}$.

No arco $\lambda$ os pontos de tangência de $Y$ com a grade são dados pelos zeros da função

$$
f(x, Y)=Q\left(x, \delta_{j}, Y\right)
$$


Aplicando o teorema da função implícita em $f$ no ponto $\left(x_{T}, X\right)$ concluímos que existe $\delta>0$ tal que todo $Y \in B_{\delta}(X)$ tem uma única tangência $p_{T}(Y)=\left(x_{T}(Y), \delta_{j}\right)$ em $\lambda$. Se $Q_{x}\left(x_{T}, \delta_{j}, X\right)>0($ respectivamente $<0)$, então $Q_{x}\left(x_{T}(Y), \delta_{j}, Y\right)>0$ (respectivamente $<0)$.

Isso é suficiente para provarmos o item $(a)$.

Vamos mostrar agora que diminuindo $\delta$ caso seja necessário, $\varphi(t, T(Y), Y)$ irá satisfazer $(b),(c)$ e $(d)$.

Considere o ponto de tangência $p_{T}=E_{j_{0}}$. Tome agora $E_{j_{0}+1}$. $\mathrm{O}$ arco de trajetória $E_{j_{0}} E_{j_{0}+1}$ está inteiramente contido em uma célula da grade $[\Gamma, \Delta]$. Sabemos que ou $E_{j_{0}+1}$ pertence a $[\Gamma, \Delta]$ ou $E_{j_{0}+1}$ pertence a $\partial \mathcal{V}_{\omega_{X}}$. Vamos supor que $E_{j_{0}+1} \in[\Gamma, \Delta]$, o outro caso é semelhante a esse.

Podemos então tomar um pequeno arco $L_{E_{j_{0}+1}}$ de $[\Gamma, \Delta]-(\Gamma \times \Delta)$ em volta de $E_{j_{0}+1}$ como seção transversal para o sistema $X$. Para valores pequenos de $\delta, L_{E_{j_{0}+1}}$ também é uma seção transversal para $Y \in B_{\delta}(X)$. Logo, pela continuidade do fluxo com relação as condições iniciais e aos campos, para todo $Y \in B_{\delta}(X), \varphi(t, T(Y), Y)$ intersecta $L_{E_{j_{0}+1}}$ em um ponto $E_{j_{0}+1}(Y)$, e o arco de trajetória $p_{T}(Y) E_{j_{0}+1}(Y)$ está contido na célula e próximo do arco de trajetória $E_{j_{0}} E_{j_{0}+1}$, não contendo pontos de esquina da grade ou outro ponto de tangência além de $p_{T}(Y)$.

Considere agora os pontos $E_{j}, j=j_{0}+1, j_{0}+2, \ldots, n$. Podemos tomar para cada ponto $E_{j}, j=j_{0}+1, j_{0}+2, \ldots, n-1$ um pequeno arco $L_{E_{j}}$ de $[\Gamma, \Delta]-(\Gamma \times \Delta)$ em volta de $E_{j}$ como seção transversal de $X$. Para $E_{n}$ tomamos $L_{E_{n}}$ um pequeno arco em $\partial \mathcal{V}_{\omega_{X}}$. Cada arco de trajetória $E_{j} E_{j+1}$ está inteiramente contido em uma célula e não contém pontos de tangência ou de esquina da grade. Podemos então, para cada um desses pontos e arcos, definir uma aplicação de transição entre as seções transversais em questão.

De fato considere $E_{j_{0}+1}$, então pela continuidade do fluxo com relação as condições iniciais e aos campos, existe $\delta_{j_{0}+1}$ e uma aplicação de transição $\Pi_{j_{0}+1}: L_{E_{j_{0}+1}} \times B_{\delta_{j_{0}+1}} \rightarrow$ $L_{E_{j_{0}+2}}$. Assim para toda órbita de $Y \in B_{\delta_{j_{0}+1}}$ passando por um ponto próximo de $E_{j_{0}+1}$ em $L_{E_{j_{0}+1}}$ ficará próximo do arco de trajetória $E_{j_{0}+1} E_{j_{0}+2}$ e irá intersectar $L_{E_{j_{0}+1}}$ em um ponto próximo de $E_{j_{0}+2}$. Em particular, se tomarmos $E_{j_{0}+2}(Y)=\Pi_{j_{0}+1}\left(E_{j_{0}+1}(Y), Y\right)$, então $E_{j_{0}+1}(Y) E_{j_{0}+2}(Y)$ é um arco de trajetória de $\varphi\left(t, p_{T}(Y), Y\right)$, que está próximo de $E_{j_{0}+1} E_{j_{0}+2}$ e não contém pontos de tangência ou esquina com a grade.

Podemos repetir o processo para todos os pontos $E_{j}$ até chegarmos em $E_{n}$, tomando o cuidado de tomarmos $\delta_{j}>0$ pequeno o suficiente para que a composição das aplicações de transição estejam bem definidas. Dessa forma, encontramos pontos $E_{j+1}(Y)=$ $\Pi_{j}\left(E_{j}(Y), Y\right)$, com $E_{j}(Y)$ próximo de $E_{j}$, e os arcos de trajetória $E_{j}(Y) E_{j+1}(Y)$ estão suficientemente próximos de $E_{j} E_{j+1}$ de forma que eles não possuam ponto de tangência ou esquina com a grade. E como $E_{n}(Y) \in \partial \mathcal{V}_{\omega_{X}}$, e $Y$ é transversal a $\partial \mathcal{V}_{\omega_{X}}$ garantimos que $\varphi\left(t, p_{T}(Y), Y\right)$ tende para um poço $\omega_{Y}$ contido em $\mathcal{V}_{\omega_{X}}$.

Podemos repetir o mesmo processo para os pontos $E_{j}, j=1,2, \ldots, j_{0}-1$, percorrendo 
a órbita no sentido negativo. Assim, como na análise anterior tomamos um número finito de $\delta^{\prime} s$, podemos escolher então $\delta>0$ pequeno o suficiente para que todo $Y \in B_{\delta}(Y)$, a órbita $\varphi\left(t, p_{T}(Y), Y\right)$ não possua pontos de esquina com a grade, tenha $p_{T}(Y)$ como seu único ponto de tangência com $[\Gamma, \Delta]$, sendo que $\varphi\left(t, p_{T}(Y), Y\right)$ liga uma fonte $\alpha_{Y} \in \mathcal{V}_{\alpha_{X}}$ com um poço $\omega_{Y} \in \mathcal{V}_{\omega_{X}}$, e intersecta o conjunto $[\Gamma, \Delta] \cup \partial \mathcal{V}_{\alpha} \cup \partial \mathcal{V}_{\omega}$ em pontos $E_{j}(Y)$, $j=1,2, \ldots, n, \operatorname{com} E_{j}(Y)$ próximo de $E_{j}$.

Isso demonstra a proposição.

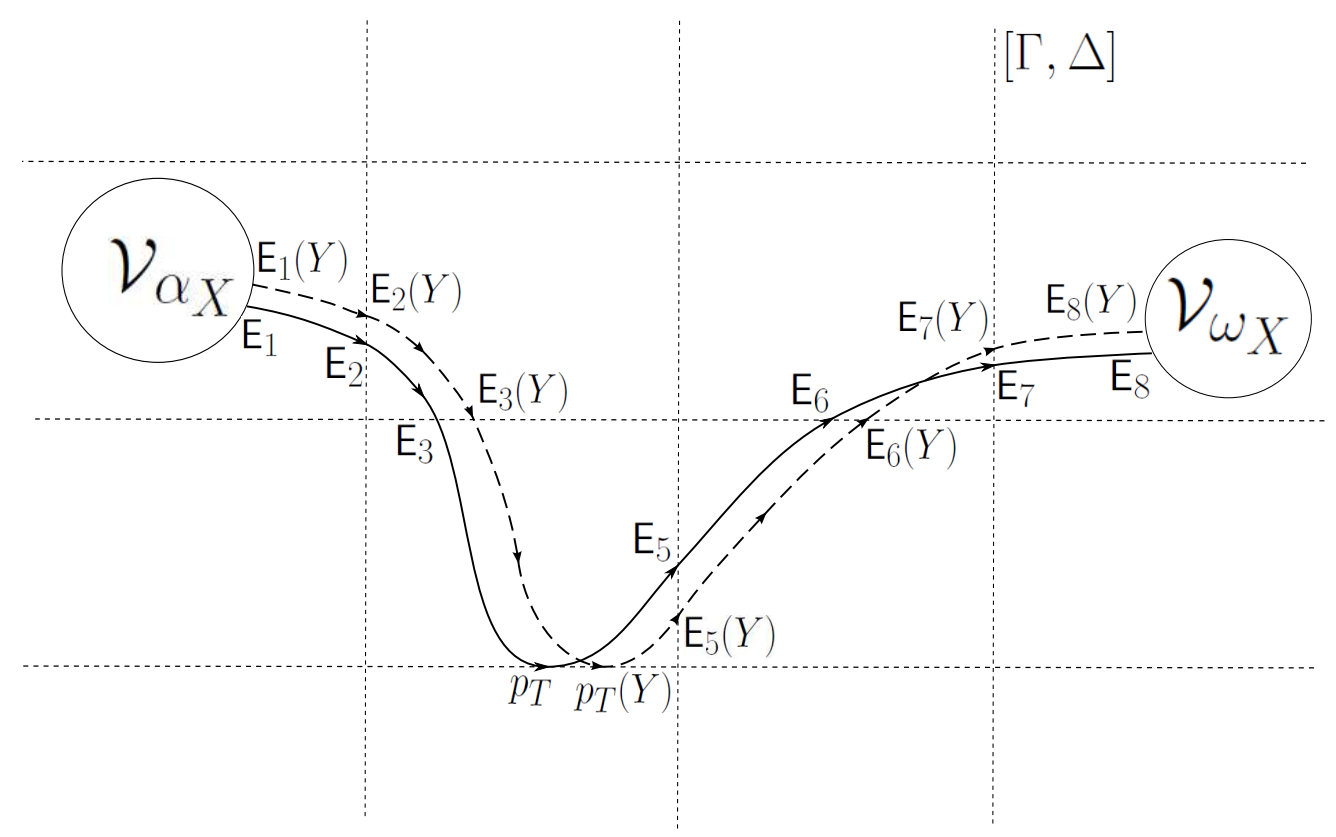

Figura 3.5: Órbitas $\varphi\left(t, p_{T}, X\right)$ e $\varphi\left(t, p_{T}(Y), Y\right)$

Lema 3.7 Seja $X \in \Sigma_{\Gamma, \Delta}$ e $e_{i j}=\left(\gamma_{i}, \delta_{j}\right)$ um ponto de esquina da grade $[\Gamma, \Delta]$. Então existe $\delta>0$ tal que todo $Y \in B_{\delta}(X)$ satisfaz as seguintes propriedades:

(a) - A órbita $\varphi\left(t, e_{i j}, Y\right)$ tem o mesmo comportamento que $\varphi\left(t, e_{i j}, X\right)$, isto é, ela intersecta a grade $[\Gamma, \Delta]$ transversalmente e percorre as mesmas células que $\varphi\left(t, e_{i j}, X\right)$;

(b) - Se $\varphi\left(t, e_{i j}, X\right)$ liga uma fonte $\alpha_{X}$ com um poço $\omega_{X}$, então $\varphi\left(t, e_{i j}, Y\right)$ liga uma fonte $\alpha_{Y}$ e um poço $\omega_{Y}$ contidos respectivamente nas vizinhanças $\mathcal{V}_{\alpha_{X}}$ e $\mathcal{V}_{\omega_{X}}$;

(c) - A órbita $\varphi\left(t, e_{i j}(Y), Y\right)$ não contém pontos de tangência com a grade $[\Gamma, \Delta]$ e contém somente $e_{i j}$ como ponto de esquina;

(d) - Se $E_{j}, j=1,2, \ldots, n$, são os pontos descritos na observação 3.4, com $E_{j_{0}}=e_{i j}$, então $\varphi\left(t, e_{i j}, Y\right)$ intersecta o conjunto $[\Gamma, \Delta] \cup \partial \mathcal{V}_{\alpha_{X}} \cup \partial \mathcal{V}_{\omega_{X}}$ em pontos $E_{j}(Y), j=$ $1,2, \ldots, n$, com $E_{j}(Y)$ próximo de $E_{j}$, e com exceção de $E_{j_{0}}(Y)=e_{i j}$, sempre o faz transversalmente e disjunto das esquinas da grade. 
Demonstração: Observe que o ponto $e_{i j}=\left(\gamma_{i}, \delta_{j}\right)$ de esquina com a grade $[\Gamma, \Delta]$ está contido no bordo de quatro células. Como $X \in \Sigma_{\Gamma, \Delta}$, sabemos que $\varphi\left(t, e_{i j}, X\right)$ para valores pequenos de $t$, deve estar contido em $\Omega_{(i-1)(j-1)} \cup \Omega_{i j}$ ou $\Omega_{(i-1) j} \cup \Omega_{i(j-1)}$. Isso ocorre pois no ponto $e_{i j}$ o campo $X$ não é tangente as retas $x=\gamma_{i}$ e $y=\delta_{j}$.

O primeiro caso ocorre quando $P\left(\gamma_{i}, \delta_{j}, X\right) Q\left(\gamma_{i}, \delta_{j}, X\right)>0$ e o segundo caso ocorre quando $P\left(\gamma_{i}, \delta_{j}, X\right) Q\left(\gamma_{i}, \delta_{j}, X\right)<0$. Logo, pela continuidade de $P$ e $Q$ com relação aos campos é claro que podemos escolher $\delta>0$ tal que a órbita $\varphi\left(t, e_{i j}, Y\right)$ de $Y \in B_{\delta_{1}}(X)$ tenha o mesmo comportamento local que $\varphi\left(t, e_{i j}, X\right)$.

A demonstração dos itens $(b),(c)$ e $(d)$ é idêntica a demonstração dos mesmos itens do lema anterior. De fato basta observar que podemos definir o ponto $E_{j_{0}+1}(Y)$ de forma análoga ao que fizemos no lema anterior, e garantimos que o arco de trajetória $e_{i j} E_{j_{0}+1}(Y)$ possui somente $e_{i j}$ como ponto de esquina, não possui pontos de tangência e intersecta $L_{E_{j_{0}+1}}$ em $E_{j_{0}+1}(Y)$ próximo de $E_{j_{0}+1}$. O resto da demonstração é análogo ao feito no lema anterior.

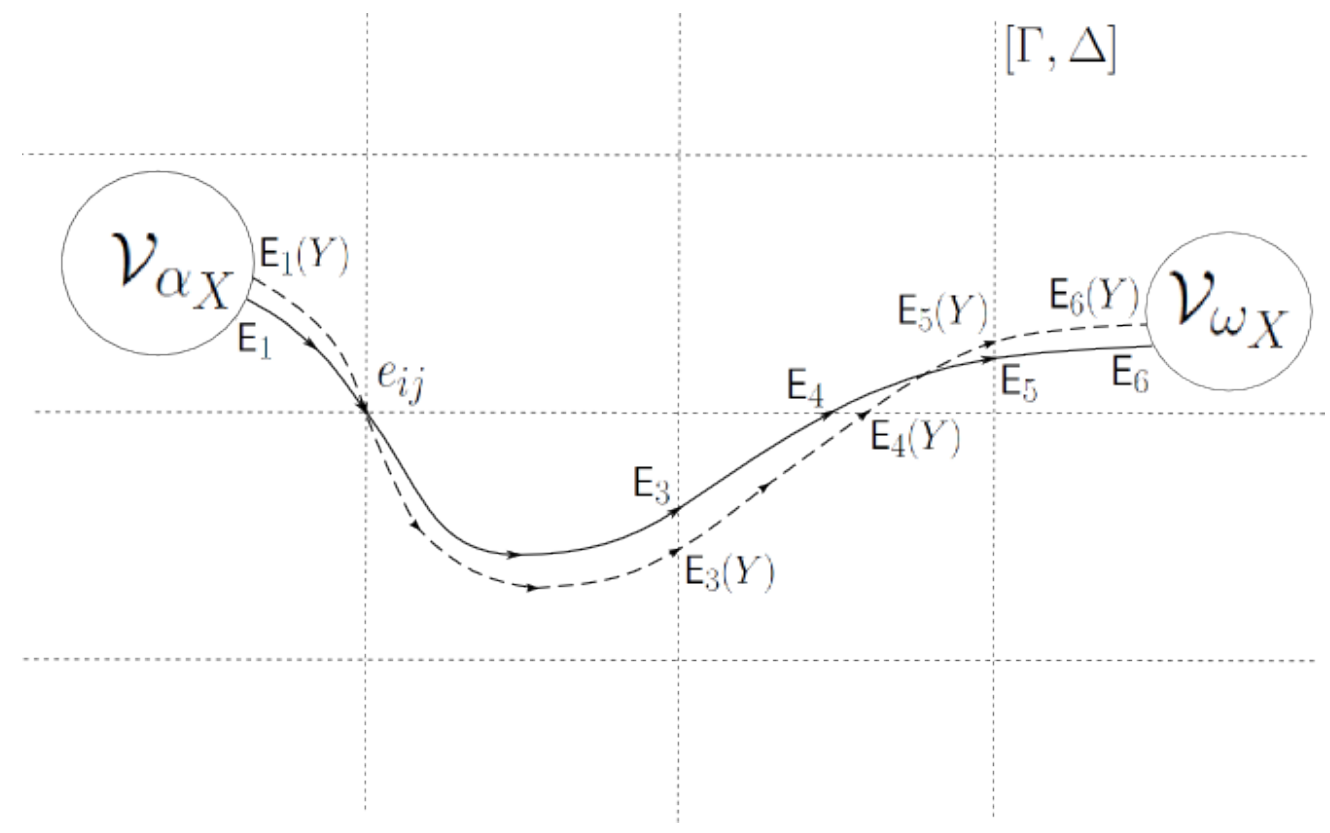

Figura 3.6: Órbitas $\varphi\left(t, e_{i j}, X\right)$ e $\varphi\left(t, e_{i j}, Y\right)$

Lema 3.8 Seja $X \in \Sigma_{\Gamma, \Delta}$ e $p_{S}$ um ponto de sela de $X$. Se $\gamma_{X}$ é uma separatriz de $p_{S}$, então existe $\delta>0$ tal que todo $Y \in B_{\delta}(X)$ satisfaz as seguintes propriedades:

(a) - Se $\gamma_{X}$ é uma separatriz instável (respectivamente estável) que tende para um poço $\omega_{X}$ (respectivamente vem de uma fonte $\alpha_{X}$ ), então a separatriz equivalente $\gamma_{Y}$ de $p_{S}(Y)$ tende para um poço $\omega_{Y}$ contido na vizinhança $\mathcal{V}_{\omega_{X}}$ (respectivamente vem de uma fonte $\alpha_{Y}$ contida na vizinhança $\mathcal{V}_{\alpha_{X}}$ ); 


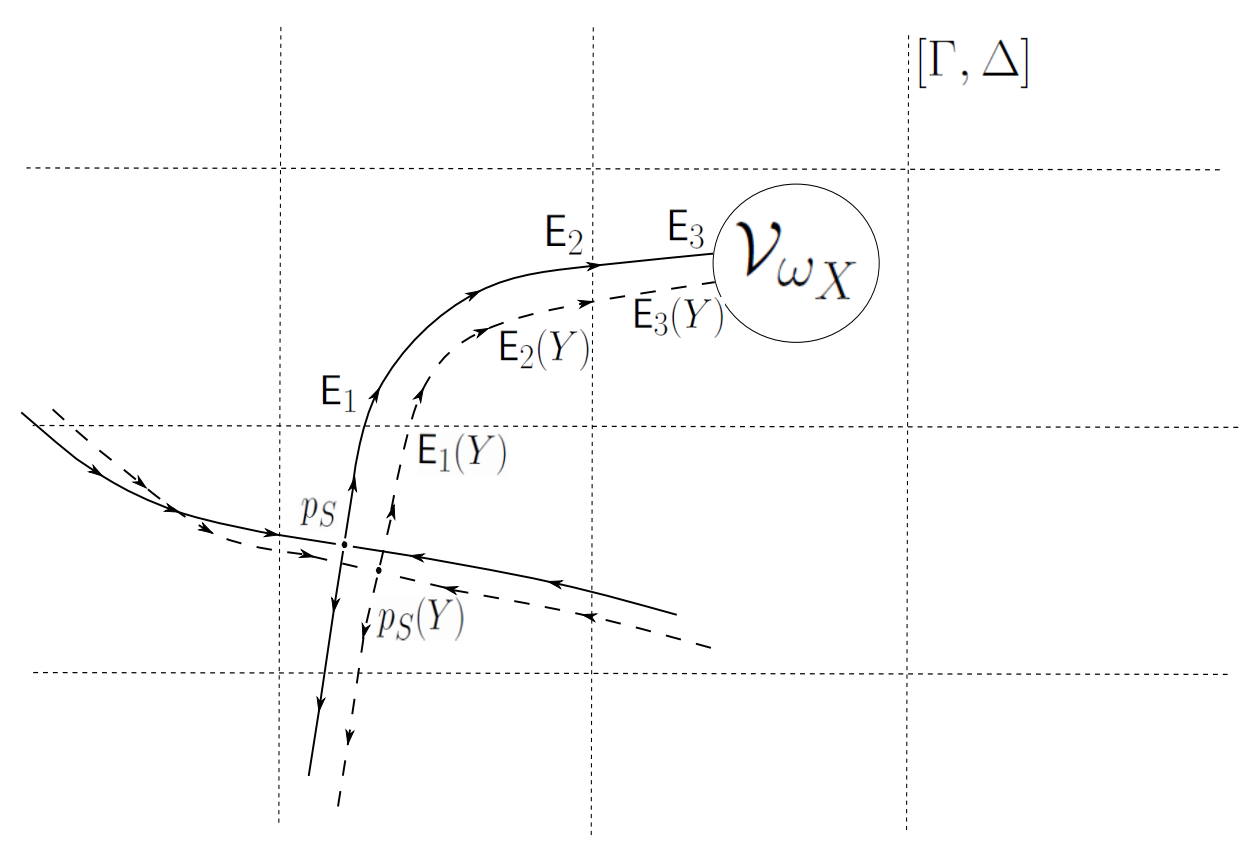

Figura 3.7: Separatrizes de sela

(b) - A órbita $\gamma_{Y}$ não contém pontos de tangência ou de esquina da grade $[\Gamma, \Delta]$;

(c) - Se $E_{j}, j=1,2, \ldots, n$, são os pontos descritos na observação 3.5, então $\gamma_{Y}$ intersecta o conjunto $[\Gamma, \Delta] \cup \partial \mathcal{V}_{\alpha_{X}} \cup \partial \mathcal{V}_{\omega_{X}}$ em pontos $E_{j}(Y), j=1,2, \ldots, n$, com $E_{j}(Y)$ próximo de $E_{j}$, sempre o faz transversalmente e disjunto das esquinas da grade.

Demonstração: Seja $X$ um campo em $\Sigma_{\Gamma, \Delta}$. Suponha que $p_{S}$ é um ponto de sela finito. Localmente as separatrizes de sela de $X$ são dadas pelos auto-espaços da matriz $A_{i j}(X)$. Por exemplo, se escrevermos a matriz

$$
A_{i j}(X)=\left(\begin{array}{ll}
a_{11}^{i j}(X) & a_{12}^{i j}(X) \\
a_{21}^{i j}(X) & a_{22}^{i j}(X)
\end{array}\right)
$$

e se supusermos $a_{12}^{i j}(X) \neq 0$, então como $A_{i j}(Y)$ depende continuamente de $Y$ podemos escrever o coeficiente angular dos auto-espaços de $A_{i j}(Y)$ como

$$
m_{i}(Y)=\frac{\left(a_{11}^{i j}(Y)-\lambda_{i}(Y)\right)}{a_{12}^{i j}(Y)},
$$

onde $\lambda_{i}, i=1,2$, são os autovalores de $A_{i j}(Y)$ que podem ser expressos por

$$
\lambda_{1,2}=\frac{\operatorname{tr} A_{i j}(Y) \pm \sqrt{\operatorname{tr} A_{i j}(Y)^{2}-4 \operatorname{det} A_{i j}(Y)}}{2 a_{12}^{i j}(Y)} .
$$

Como essas funções são todas contínuas, dada uma separatriz $\gamma_{X}$ que intercepta um 
lado da grade $\Omega_{i j}$ num ponto $E_{1}$, então para $\delta$ suficientemente pequeno, a separatriz equivalente de $Y$ interceptará o mesmo lado da grade em um ponto $E_{1}(Y)$ próximo de $E_{1}$.

O mesmo acontece para separatrizes de pontos de sela no infinito, pois basta observarmos que essas separatrizes são dadas pelos auto-espaços de nós na célula infinita que contém $p_{S}$. Logo podemos fazer a mesma análise que no caso de uma célula finita, pois esses auto-espaços dependem continuamente de $Y$.

O resto da demonstração é semelhante a dos lemas anteriores, pois basta compormos as aplicações de transição entre os lados da grade $[\Gamma, \Delta]$ até chegarmos em $\mathcal{V}_{\omega_{X}}$ ou $\mathcal{V}_{\alpha_{\mathcal{X}}}$. 


\section{Capítulo 4}

\section{Abertura, Densidade e Estabilidade Estrutural de $\Sigma_{\Gamma, \Delta}$}

Neste capítulo demonstraremos o teorema 3.1, o qual é o resultado principal deste trabalho.

Dividiremos sua demonstração em três partes: primeiro vamos demonstrar que o conjunto $\Sigma_{\Gamma, \Delta}$ é aberto em $\mathfrak{X}_{\Gamma, \Delta}$; depois vamos, através do método das regiões canônicas, construir os homeomorfismos necessários para mostrarmos que os campos que estão em $\Sigma_{\Gamma, \Delta}$ são estruturalmente estáveis; e por fim vamos demonstrar o teorema 3.3, o que implicará que o conjunto $\Sigma_{\Gamma, \Delta}$ é denso em $\mathfrak{X}_{\Gamma, \Delta}$.

Usaremos os métodos assimilados de [SG03, PP59, Sot81, Sot85, Duf53], com as alterações e observações necessárias para sua aplicação aos campos aqui considerados.

\subsection{Abertura}

Proposição 4.1 O conjunto $\Sigma_{\Gamma, \Delta}=\Sigma_{\Gamma, \Delta}(1) \cap \Sigma_{\Gamma, \Delta}(2) \cap \Sigma_{\Gamma, \Delta}(3)$ é aberto em $\mathfrak{X}_{\Gamma, \Delta}$.

Demonstração: É uma consequência direta dos lemas 3.1, 3.2, 3.3, 3.4, 3.5, 3.6, 3.7 e 3.8. De fato, dado $X \in \Sigma_{\Gamma, \Delta}$, basta tomarmos $\delta>0$ pequeno o suficiente para que os lemas mencionados anteriormente sejam válidos, garantindo assim que todo $Y \in B_{\delta}(X)$ satisfaz as condições para estar no conjunto $\Sigma_{\Gamma, \Delta}$.

\subsection{Regiões canônicas}


Seja $X \in \Sigma_{\Gamma, \Delta}$ e considere o campo de vetores compactificado $X_{\mathbb{D}}$.

Definição 4.1 Uma trajetória $\gamma$ do campo de vetores $X \in \Sigma_{\Gamma, \Delta}$ é dita uma separatriz do sistema se ela for de um dos seguintes tipos:

(a) Ponto singular,

(b) Órbita periódica,

(c) Trajetória contendo um ponto de tangência com a grade $[\Gamma, \Delta]$,

(d) Trajetória contendo um ponto de esquina da grade $[\Gamma, \Delta]$,

(e) Separatrizes de sela,

(f) Trajetória no infinito.

Dizemos que uma trajetória de $X$ é ordinária se ela não for uma separatriz. Chamaremos de $S_{X}$ o conjunto fechado em $\mathbb{D}$ formado pelo fecho da união de todas as separatrizes de $X$.

Definição 4.2 Dizemos que uma sub-região $R$ de $\mathbb{D}$ é uma região paralela relativa ao campo $X$ se o conjunto de trajetórias de $X_{\mathbb{D}}$ em $R$ é equivalente a um dos casos seguintes:

(i) O conjunto de segmentos de reta horizontais preenchendo uma faixa vertical aberta, semi-aberta ou fechada, limitada por duas linhas paralelas,

(ii) O conjunto de todos os raios em um disco aberto ou fechado, excluindo o centro,

(iii) O conjunto de circunferências concêntricas, excluindo o centro.

Sejam agora $\mathcal{V}_{p_{E_{1}}}, \mathcal{V}_{p_{E_{2}}}, \ldots, \mathcal{V}_{p_{E_{k}}}$ as vizinhanças dos pontos singulares hiperbólicos atratores ou repulsores de $X$ dadas pelos lemas 3.1 e 3.3 e $\mathcal{V}_{\gamma_{1}}, \mathcal{V}_{\gamma_{2}}, \ldots, \mathcal{V}_{\gamma_{l}}$ as vizinhanças da órbitas periódicas hiperbólicas de $X$ dadas pelos lemas 3.2 e 3.4 .

Considere $D$ o conjunto fechado formado retirando-se de $\mathbb{D}$ o interior das vizinhanças $\mathcal{V}_{p_{E_{r}}}$ e $\mathcal{V}_{\gamma_{s}}$. Tome também o conjunto aberto $D^{\prime}=\stackrel{\circ}{D} \backslash S_{X}$.

Com essa notação introduzimos a seguinte definição.

Definição $4.3 R$ é uma região canônica de $X$ se for a intersecção de uma componente conexa do aberto $D^{\prime}$ com o interior de uma célula $\Omega_{i j}$. 
Observação 4.1 A nossa definição de região canônica é baseada na definição de região canônica que aparece no artigo [PP59]. Acrescentamos somente a exigência de que a região canônica $R$ esteja inteiramente contida em alguma célula $\Omega_{i j}$. Isso porquê devemos levar em consideração que a grade $[\Gamma, \Delta]$ deve ser invariante pelo homeomorfismo que dá a equivalência topológica entre os campos. Dessa forma as regiões canônicas que aparecem nesse trabalho são as mesmas existentes no caso de campos de vetores de classe $C^{1}$ se considerarmos que o conjunto compacto onde o campo está definido é uma célula da grade compactificada $[\Gamma, \Delta]$.

Observação 4.2 É fácil ver que o interior das regiões canônicas que aparecem associadas aos campos em $\Sigma_{\Gamma, \Delta}$ são somente regiões paralelas do tipo $(i)$ e $(i i)$.

Chamaremos de conjunto $\alpha_{R}$ o arco contido em $[\Gamma, \Delta]$ ou em $\mathcal{V}_{\alpha}$, onde $\alpha$ é uma fonte do campo de vetores, por onde as órbitas de $X$ entram na região canônica $R$.

De forma semelhante chamaremos de conjunto $\omega_{R}$ o arco contido em $[\Gamma, \Delta]$ ou $\mathcal{V}_{\omega}$, onde $\omega$ é um poço do campo de vetores, por onde as órbitas de $X$ deixam a região canônica $R$.

Esses arcos são determinados pelas separatrizes $S_{X}$ do sistema, logo é fácil observar que para toda região canônica $R$ não existem pontos de alguma separatriz de $X$ no interior dos conjuntos $\alpha_{R}$ ou $\omega_{R}$.

Listaremos agora os possíveis tipos de regiões canônicas de $X$ em $\mathbb{D}$. Da forma como essas regiões foram definidas, fica claro que a fronteira de cada região canônica possui exatamente um conjunto $\alpha_{R}$ e um conjunto $\omega_{R}$. Além disso, como $X$ está em $\Sigma_{\Gamma, \Delta}$, sabemos que em cada célula finita o campo de vetores possui no máximo um ponto singular e ainda, em cada célula infinita, podemos ter no máximo um ponto de sela. Logo o bordo de uma região canônica $R$ do campo de vetores $X$ poderá ter no máximo um ponto de sela.

Podemos portanto classificar as regiões canônicas de acordo com o número e tipos de separatrizes em seu bordo. Os possíveis tipos de regiões canônicas estão esquematizados na figura 4.1 e descritos mais adiante. Nas figuras $p_{S}$ representa um ponto de sela e em todos os casos os conjuntos $\alpha_{R}$ e $\omega_{R}$ podem ser permutados.m

\section{Possíveis regiões canônicas:}

I - A fronteira de $R$ consiste de um conjunto $\alpha_{R}$ e um conjunto $\omega_{R}$. Exemplo: (a) Se o sistema possui apenas um ponto singular que é um foco e a grade $[\Gamma, \Delta]$ é vazia, então $\alpha_{R}$ e $\omega_{R}$ são os bordos das vizinhanças do foco e da órbita no infinito.

II - A fronteira de $R$ consiste de uma sela $p_{S}$ com duas separatrizes tendendo a ela, um conjunto $\alpha_{R}$, um conjunto $\omega_{R}$ e um arco de separatriz $\gamma$, que pode ser somente um ponto. Exemplos: (a) Em uma célula $\Omega_{i j}$ temos um ponto de sela $p_{S}$, a separatriz 

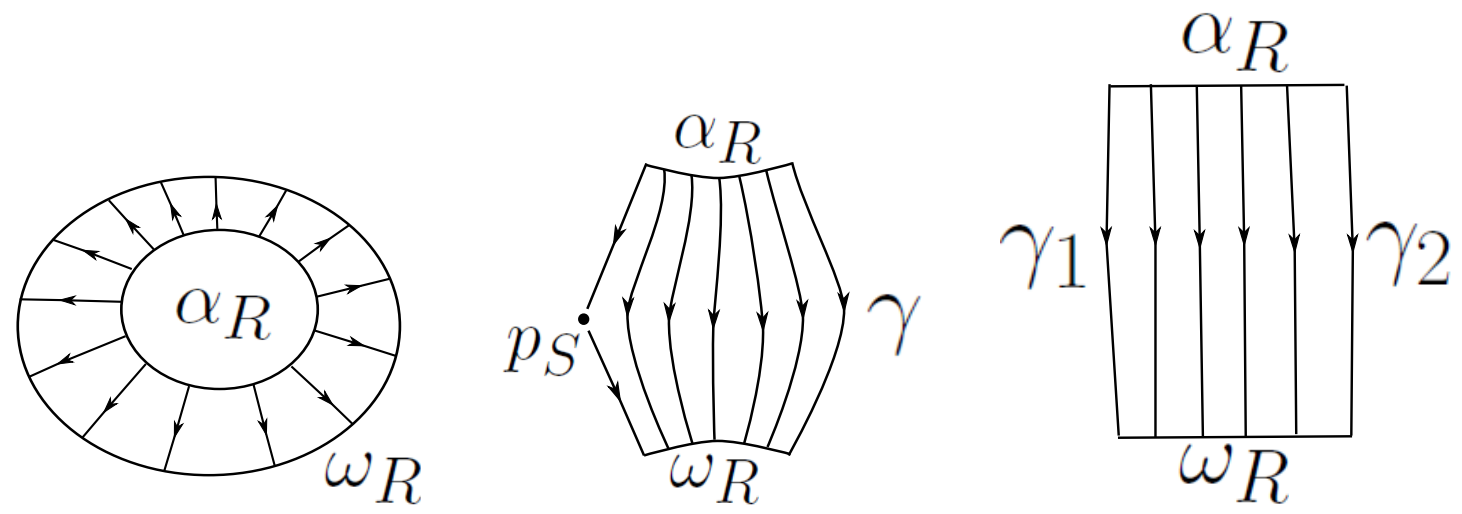

Figura 4.1: Regiões canônicas esquematizadas do tipo I, II e III respectivamente

$\gamma$ é um ponto de tangência com a grade $[\Gamma, \Delta], \alpha_{R}$ e $\omega_{R}$ são arcos na fronteira de $\Omega_{i j}$. (b) Em uma célula $\Omega_{i j}$ temos um ponto sela $p_{S}$, a separatriz $\gamma$ é uma órbita passando por uma esquina da grade $[\Gamma, \Delta], \alpha_{R}$ e $\omega_{R}$ são arcos na fronteira de $\Omega_{i j}$.

(c) Em uma célula infinita $\Omega_{i j}$ temos um ponto de sela $p_{S}$, a separatriz $\gamma$ é um arco no infinito, $\alpha_{R}$ e $\omega_{R}$ são nós no infinito.
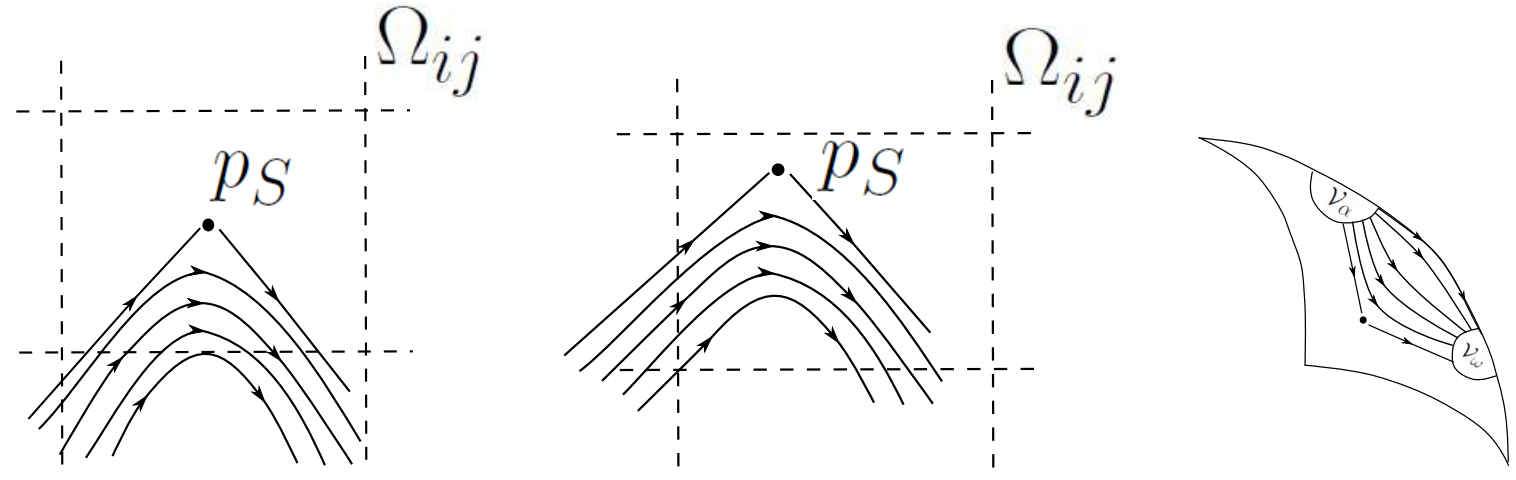

Figura 4.2: Exemplos regiões canônicas tipo II

III - A fronteira consiste de um conjunto $\alpha_{R}$, um conjunto $\omega_{R}$ e arcos de duas separatrizes $\gamma_{1}$ e $\gamma_{2}$. Exemplos: (a) A fronteira da região tem duas órbitas passando por esquinas de $\Omega_{i j}$ e tendendo para um poço, sendo $\alpha_{R}$ um arco da fronteira de $\Omega_{i j}$ e $\omega_{R}$ um arco na vizinhança do poço. (b) A fronteira da região tem duas órbitas tangentes a grade em $\Omega_{i j}$ e tendendo para um poço, sendo $\alpha_{R}$ um arco da fronteira de $\Omega_{i j}$ e $\omega_{R}$ um arco na vizinhança do poço.

Dado um campo $X$ em $\Sigma_{\Gamma, \Delta}$, tome $S_{X}$ o conjunto de todas as separatrizes de $X$. Para todo $Y$ próximo de $X$ considere $S_{Y}$ seu conjunto de separatrizes. Vale então o seguinte teorema.

Teorema 4.1 Se $\delta>0$ é suficientemente pequeno e $Y \in B_{\delta}(X)$, então existe uma correspondência biunívoca entre os conjuntos $S_{X}$ e $S_{Y}$ de forma que: 


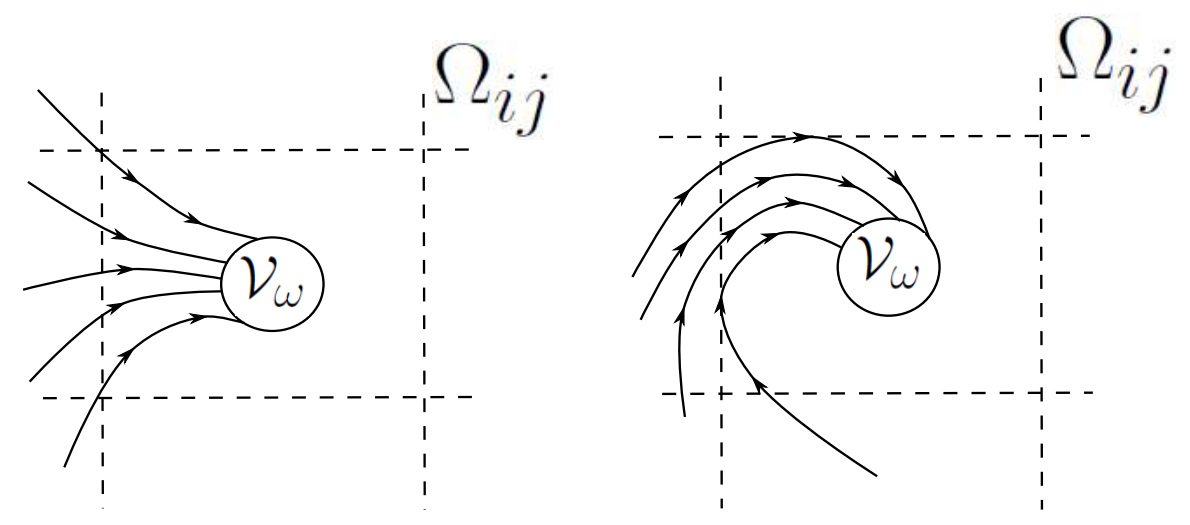

Figura 4.3: Exemplos regiões canônicas tipo III

- Separatrizes correspondentes são do mesmo tipo,

- Um subconjunto de $S_{X}$ limita uma região canônica de $X$ se e somente se o subconjunto correspondente de $S_{Y}$ limita uma região canônica equivalente em $Y$.

Demonstração: Isso é uma consequência dos lemas 3.1, 3.2, 3.3, 3.4, 3.5, 3.6, 3.7 e 3.8. De fato, suponha $\delta>0$ pequeno o suficiente tal que os lemas sejam válidos para todos os pontos singulares de $X$, finitos ou infinitos, todas as órbitas periódicas, todos os pontos de tangência e esquina com a grade $[\Gamma, \Delta]$. Tome agora um campo de vetores $Y \in B_{\delta}(X)$.

Pelos lemas 3.1, 3.2, 3.3, 3.4 e 3.5, nós garantimos a correspondência um a um entre os conjuntos de pontos singulares e órbitas periódicas de $X$ e $Y$. Esta correspondência preserva o tipo e a estabilidade desses pontos e órbitas, bem como a maneira que esses conjuntos intersectam a grade $[\Gamma, \Delta]$.

Os lemas 3.6, 3.7 e 3.8 garantem a correspondência entre as separatrizes de $X$ e de $Y$ que são do mesmo tipo, isto é, as separatrizes que tendem para pontos de sela, possuem pontos de tangência ou passam por pontos de esquina com a grade. Essa correspondência é tal que separatrizes associadas conectam fontes, poços e selas associadas, e cortam a grade $[\Gamma, \Delta]$ de uma forma equivalente.

Daí é claro que se algum conjunto limita uma região canônica $R_{X}$ de $X$, então as separatrizes correspondentes de $Y$ limitam uma região canônica $R_{Y}$ equivalente. Isso demonstra o teorema.

De fato, as regiões canônicas junto com as vizinhanças das fontes e dos poços de $X$ particionam $\mathbb{D}$ em um número finito de sub-regiões. Pelo teorema anterior, se $Y$ estiver próximo o suficiente de $X$, então as regiões canônicas de $Y$ e as vizinhanças das fontes e poços particionam $\mathbb{D}$ em sub-regiões equivalentes às de $X$, inclusive em quantidade. Logo para construirmos o homeomorfismo $h_{Y}$ entre os campos de vetores $X$ e $Y$ basta construirmos os homeomorfismos para as vizinhanças das fontes e dos poços e para as 
regiões canônicas equivalentes dos campos.

Porém, devemos tomar o cuidado para estendermos coerentemente esses homeomorfismos para os bordos dessas regiões de modo que quando juntarmos todos eles consigamos um homeomorfismo $h_{Y}: \mathbb{D} \rightarrow \mathbb{D}$.

É importante notar que, quando temos um campo de vetores $X$ e duas regiões canônicas adjacentes, então é possível que o conjunto $\omega$ de uma região seja também o conjunto $\alpha$ da outra região. Assim devemos tomar o cuidado de construirmos os homeomorfismos na ordem correta.

Por exemplo, se $R$ e $\tilde{R}$ são duas regiões canônicas de $X$ e $\omega_{R}=\alpha_{\tilde{R}}$, então devemos primeiro construir o homeomorfismo para a região $R$ e somente depois construir o homeomorfismo para a região $\tilde{R}$, levando em consideração que os valores do homeomorfismo em $\alpha_{\tilde{R}}$ já estão determinados e são iguais aos valores em $\omega_{R}$ do homeomorfismo definido na região canônica $R$.

A figura 4.4 ilustra algumas regiões canônicas adjacentes. Para definirmos os homeomorfismos para as regiões ilustradas na figura devemos primeiro construir o homeomorfismo para a região adjacente a $\mathcal{V}_{\alpha}$ e prosseguirmos sucessivamente até definirmos o homeomorfismo para a região adjacente a $\mathcal{V}_{\omega}$.

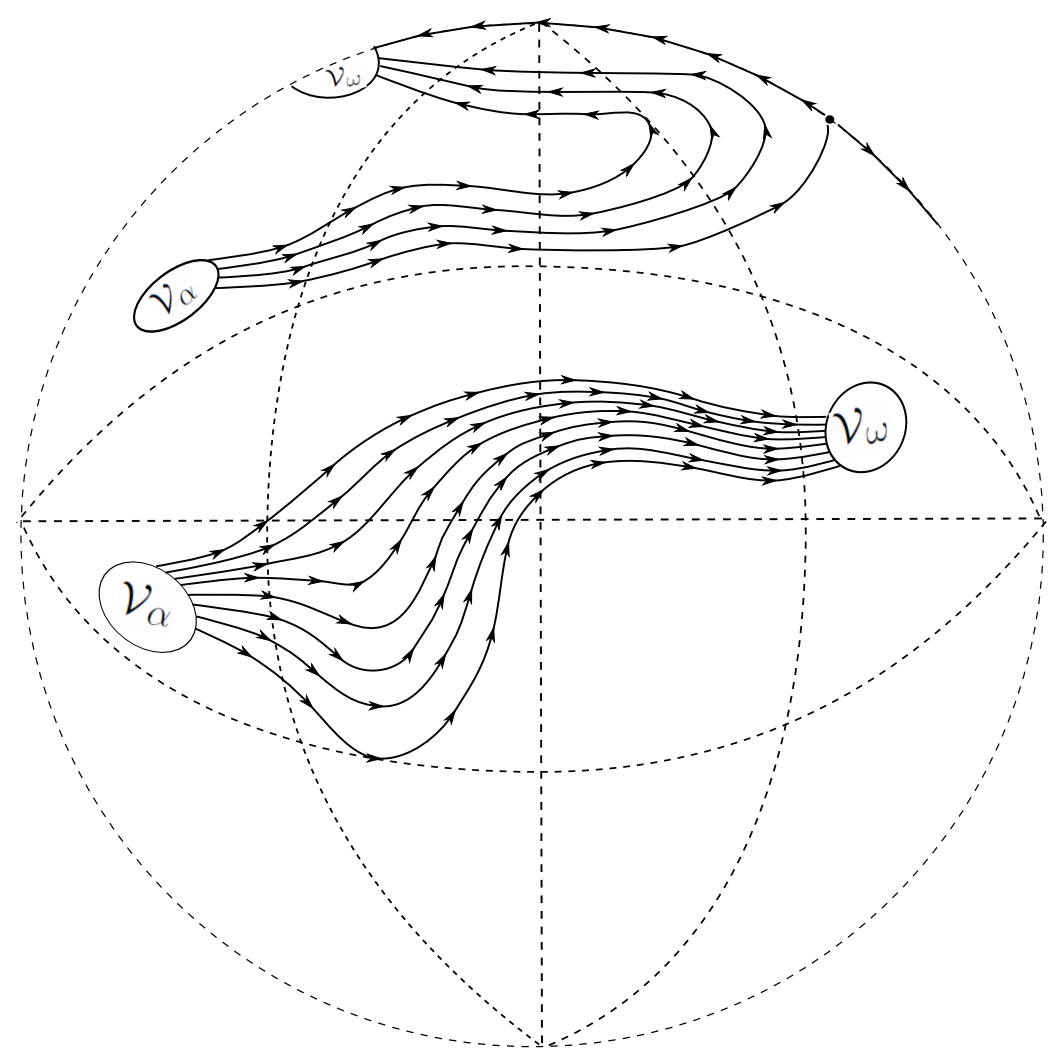

Figura 4.4: Regiões canônicas adjacentes 


\subsubsection{Construção do homeomorfismo}

Para a construção do homeomorfismo precisaremos dos seguintes lemas.

Lema 4.1 Seja $p_{S}$ um ponto de sela de $X_{\mathbb{D}}$ para o qual tendem duas separatrizes $\gamma_{1}$ $(t \rightarrow \infty)$ e $\gamma_{2}(t \rightarrow-\infty)$. Nos pontos $M_{1} \in \gamma_{1} e M_{2} \in \gamma_{2}$ considere duas seções transversais, $L_{1}$ e $L_{2}$. Chame de $N_{2}$ o ponto onde a trajetória através de $N_{1} \in L_{1}$ intersecta $L_{2}$. Então quando $N_{1} \rightarrow M_{1}$, temos que $\left|N_{1} N_{2}\right| \rightarrow\left|M_{1} p_{S}\right|+\left|p_{S} M_{2}\right|$.

Demonstração: A demonstração desse lema pode ser vista em [PP59].

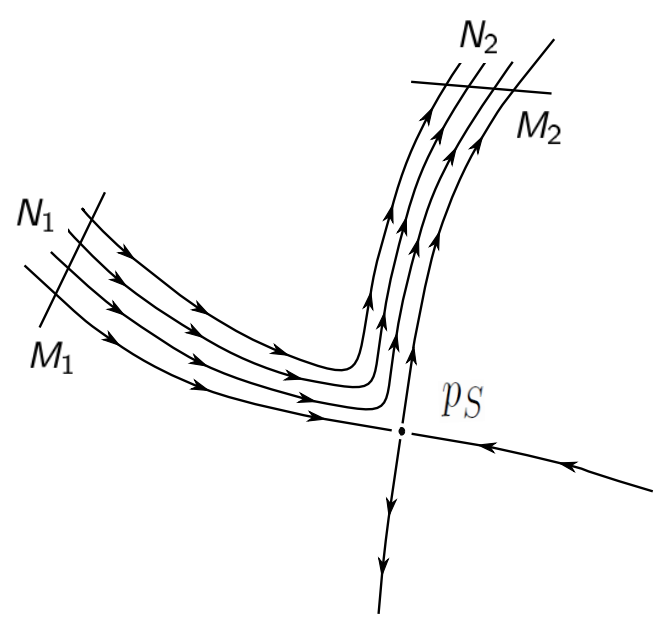

Lema 4.2 Seja $A B$ um arco e $\left(A_{n} B_{n}\right)_{n \in \mathbb{N}}$ uma sequência de arcos convergindo uniformemente para $A B$ de forma que $\left|A_{n} B_{n}\right| \rightarrow|A B|$ quando $n \rightarrow \infty$. Então:

(a)- Uma sequência de pontos $M_{n} \in A_{n} B_{n}$ com $z_{n}=\frac{A_{n} M_{n}}{A_{n} B_{n}}$ converge para um ponto $M \in A B$ se, e somente se, $z_{n} \rightarrow z=\frac{A M}{A B}$,

(b)- Os pontos em $A_{n} B_{n}$ convergem uniformemente para pontos em AB com iguais coordenadas z.

Demonstração: A demonstração desse lema pode ser vista em [PP59].

Proposição 4.2 Seja $X \in \Sigma_{\Gamma, \Delta}$. Então existe $\delta>0$ tal que se $Y \in B_{\delta}(X)$, existe um homeomorfismo $h_{Y}: \mathbb{D} \longrightarrow \mathbb{D}$ que transforma trajetórias de $X$ em trajetórias de $Y$ preservando a grade compactificada $[\Gamma, \Delta]$ e a fronteira $\partial \mathbb{D}$. 
Demonstração: Considere um campo de vetores $X \in \Sigma_{\Gamma, \Delta}$ fixado e seja $\delta>0$ suficientemente pequeno para que o teorema 4.1 seja satisfeito. Vamos construir o homeomorfismo $h_{Y}$ para todo $Y \in B_{\delta}(X)$.

Observe que, pelo teorema 4.1, existe uma correspondência um a um entre as regiões canônicas de $X$ e $Y$. Como todas essas regiões canônicas são regiões paralelas é claro que existe um homeomorfismo que preserva trajetórias transformando uma região em outra. Mas, como observado anteriormente, precisamos estender coerentemente estes homeomorfismos para os respectivos bordos dessas regiões para obtermos $h_{Y}$.

Primeiro vamos construir o homeomorfismo no conjunto fechado $D$.

Para isso sejam $R_{X}$ e $R_{Y}$ duas regiões canônicas correspondentes de $X$ e $Y$. Construiremos o homeomorfismo $f: R_{X} \rightarrow R_{Y}$.

Faremos isso para cada tipo de região canônica que apresentamos anteriormente.

Primeiro suponha que $R_{X}$ é uma região canônica do tipo I. Nesse caso temos $R_{X}=$ $R_{Y}$. Essa região é limitada por duas curvas fechadas $\alpha_{R}$ e $\omega_{R}$ que são curvas limitando vizinhanças de uma fonte e um poço respectivamente.

Tome agora um homeomorfismo $g$ de $\alpha_{R}$ em um círculo, isto é, $0 \leqslant u \leqslant 1$ com os pontos 0 e 1 identificados. Então para todo $M$ em $R_{X}, M \in A B$, nos fazemos $\Phi_{X}(M)=(u, v)$, onde $u=g(A) \mathrm{e}$

$$
v=\frac{|A M|}{|A B|}
$$

Isto define um homeomorfismo de $R_{X}$ no produto do círculo pelo intervalo $0 \leqslant v \leqslant 1$. Fazendo o mesmo para $R_{Y}$ e identificando os pontos com mesma coordenada $(u, v)$ temos o homeomorfismo desejado $f: R_{X} \rightarrow R_{Y}$.

Suponha agora que $R_{X}$ é uma região canônica do tipo II. Então $R_{X}$ será limitado por duas curvas $A_{0} p_{X} B_{0}$ e $A_{1} B_{1}$, sendo a primeira formada por uma sela $p_{X}$ e dois arcos de separatrizes, e a segunda sendo uma separatriz do campo $X$. O resto da fronteira de $R_{X}$ é formado por um conjunto $\alpha_{R_{X}}=A_{0} A_{1}$ e por um conjunto $\omega_{R_{X}}=B_{0} B_{1}$, cada um sendo um arco de $[\Gamma, \Delta]$ ou de uma curva que limita uma vizinhança de um poço ou uma fonte.

De forma análoga, $R_{Y}$ será limitado por duas curvas $\tilde{A}_{0} p_{Y} \tilde{B}_{0}$ e $\tilde{A}_{1} \tilde{B}_{1}$, sendo $p_{Y}$ um ponto de sela, e por conjuntos $\alpha_{R_{Y}}=\tilde{A}_{0} \tilde{A}_{1}$ e $\omega_{R_{Y}}=\tilde{B}_{0} \tilde{B}_{1}$ que são arcos das mesmas curvas que $\alpha_{R_{X}}$ e $\omega_{R_{X}}$ respectivamente.

Para a construção do homeomorfismo, vamos supor que nos segmentos $A_{1} B_{1}$ e $\tilde{A}_{1} \tilde{B}_{1}$ nos temos dois pontos de tangência com a grade $t$ e $\tilde{t}$ respectivamente. Os outros casos que aparecem em regiões canônicas do tipo II são tratados de forma semelhante.

Definimos então uma aplicação $\Phi_{X}: R_{X} \rightarrow U, U=\{(u, v) \mid 0 \leqslant u \leqslant 1 e 0 \leqslant v \leqslant 2\}$, da seguinte maneira:

O arco $A_{0} A_{1}$ é levado em $\{(u, 0) \mid 0 \leqslant u \leqslant 1\}$ por um homeomorfismo $g$. Assim se $A \in A_{0} A_{1}, \Phi_{X}(A)=(g(A), 0)$.

Estenda agora $\Phi_{X}$ de forma que o arco $A_{0} p_{X}$ seja levado em $\{(0, v) \mid 0 \leqslant v \leqslant 1\}$ por 


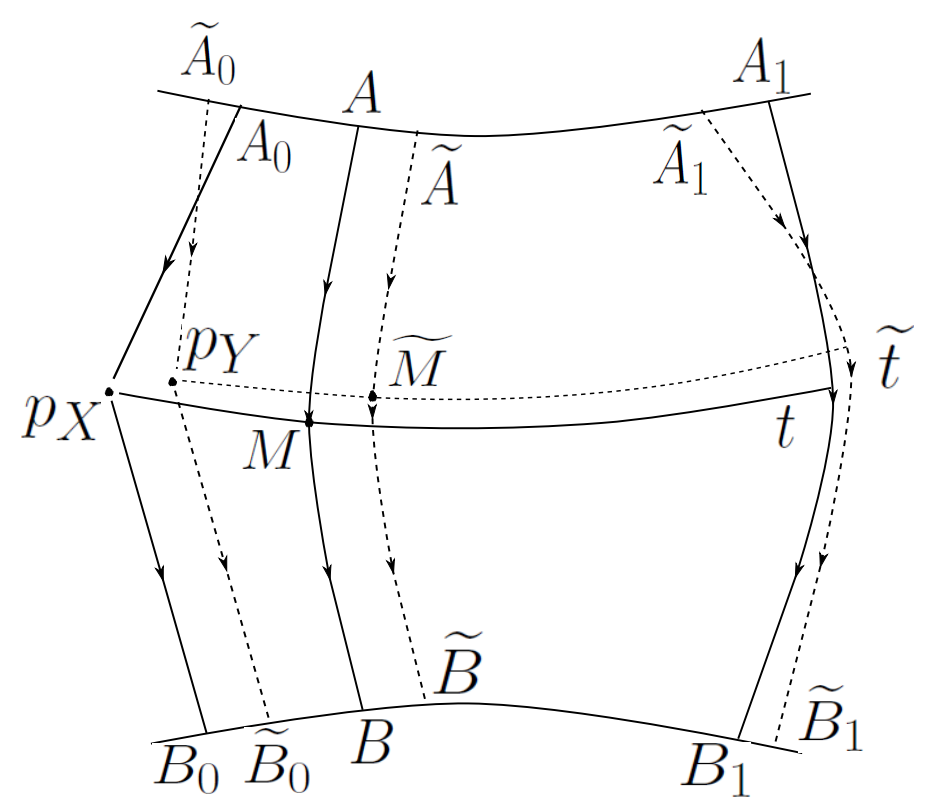

razão do comprimento de arco. Ou seja, se $A \in A_{0} p_{X}$, então $\Phi_{X}(A)=(0, v)$, onde

$$
v=\frac{\left|A_{0} A\right|}{\left|A_{0} p_{X}\right|} .
$$

Da mesma forma estenda $\Phi_{X}$ por razão do comprimento de arco tal que $\Phi_{X}\left(p_{X} B_{0}\right)=$ $(0,1 \leqslant v \leqslant 2), \Phi_{X}\left(A_{1} t\right)=(1,0 \leqslant v \leqslant 1)$ e $\Phi_{X}\left(t B_{1}\right)=(1,1 \leqslant v \leqslant 2)$.

Para estender $\Phi_{X}$ para as partes restantes de $R_{X}$, seja $\mu_{0}=\frac{\left|A_{0} p_{X}\right|}{\left|A_{0} B_{0}\right|}$ e $\mu_{1}=\frac{\left|A_{1} t\right|}{\left|A_{1} B_{1}\right|}$. Em cada trajetória $A B$ começando em um ponto $A$ tal que $g(A)=u$, consideramos um ponto $M$ tal que

$$
\frac{|A M|}{|A B|}=(1-u) \mu_{0}+u \mu_{1}
$$

e definimos $\Phi_{X}(M)=(u, 1)$.

Se $P \in A B$ definimos $\Phi_{X}(P)=(u, v)$, onde

$$
v=\frac{|A P|}{|A M|}
$$

quando $P \in A M$ e

$$
v=1+\frac{|M P|}{|M B|}
$$

quando $P \in M B$.

Como por todo ponto de $R_{X}$ onde $\Phi_{X}$ não estava definido passa uma trajetória $A B$, $\Phi_{X}$ fica então definida em todo $R_{X}$.

Claramente $\Phi_{X}$ é bijetora. A continuidade de $\Phi_{X}$ em $R_{X}$ segue da continuidade do fluxo com relação às condições iniciais junto com os lemas 4.2 e 4.1. Da mesma forma podemos concluir que $\Phi_{X}^{-1}$ é contínua, sendo portanto $\Phi_{X}$ um homeomorfismo entre $R_{X}$ e $U$. 
Analogamente podemos construir um homeomorfismo $\Phi_{Y}: R_{Y} \rightarrow U$. Portanto se tomarmos $f=\Phi_{Y}^{-1} \circ \Phi_{X}: R_{X} \longrightarrow R_{Y}$ temos o homeomorfismo desejado entre $R_{X}$ e $R_{Y}$.

As regiões canônicas do tipo III podem ser tratadas da mesma forma que as regiões do tipo II. De fato, dada a construção que fizemos para as regiões do tipo II, fica claro como devem ser construído o homeomorfismo para tais regiões.

Da forma como os homeomorfismos foram construídos, dadas duas regiões canônicas adjacentes, fica claro que as funções são iguais na parte comum de seus domínios de definição. De fato, se os homeomorfismos forem construídos na ordem correta as funções coincidem nos conjuntos $\alpha$ e $\omega$ que pertencem a mais de uma região canônica, além disso, nos arcos das separatrizes do campo sempre definimos as funções pela razão de comprimento de arco.

Isso garante que, quando juntamos todos os homeomorfismos definidos nas regiões canônicas dos campos $X$ e $Y$, obtemos um homeomorfismo $f_{D}: D \rightarrow D$ que leva órbitas de $X$ em órbitas de $Y$ e preserva a grade compactificada e o conjunto $\partial \mathbb{D}$.

Devemos agora definir o homeomorfismo $f$ para as vizinhanças $\mathcal{V}_{\alpha}$ e $\mathcal{V}_{\omega}$ associadas a fontes e poços do campo de vetores.

Sem perda de generalidade faremos as construções dos homeomorfismos supondo que $\mathcal{V}_{\omega}$ é vizinhança de um poço. A construção para as vizinhanças das fontes é análoga. As seguintes construções se aplicam tanto para poços finitos quanto para poços infinitos.

Suponha que o poço $\omega=p_{X}$ é um ponto singular atrator de $X$, portanto um nó ou um foco atrator.

Seja $p_{Y}$ o ponto singular atrator de $Y$ em $\mathcal{V}_{p_{X}}$. Tome o homeomorfismo $f_{D}$ restrito a $\partial \mathcal{V}_{\omega}$

Claramente, todo ponto $M$ de $\mathcal{V}_{\omega} \backslash\left\{p_{X}\right\}$ está contido em uma órbita $\varphi(t, p, X), 0 \leqslant$ $t<\infty, p \in \partial \mathcal{V}_{p_{X}}, \operatorname{com} \lim _{t \rightarrow \infty} \varphi(t, p, X)=p_{X}$.

O mesmo vale para o campo $Y$ em $\mathcal{V}_{\omega} \backslash\left\{p_{Y}\right\}$.

Defina então $f: \mathcal{V}_{\omega} \rightarrow \mathcal{V}_{\omega}$ da seguinte maneira:

$$
f\left(p_{X}\right)=p_{Y}
$$

e se $M=\varphi(t, p, X)$, então

$$
f(M)=\varphi\left(t, f_{D}(p), Y\right) .
$$

Claramente $f$ é uma função sobrejetora em $\mathcal{V}_{\omega}$. A continuidade de $f$ em $\mathcal{V}_{\omega} \backslash\left\{p_{X}\right\}$ e de $f^{-1}$ em $\mathcal{V}_{\omega} \backslash\left\{p_{Y}\right\}$ é consequência da dependência contínua do fluxo com relação às condições iniciais e aos campos.

Falta apenas mostrar que $f$ é continua em $p_{X}$. De fato, suponha que isso não fosse verdade. 
Então existiria uma sequência de pontos $\left(M_{n}\right)_{n \in \mathbb{N}}$ tal que

$$
M_{n} \underset{n \rightarrow \infty}{\longrightarrow} p_{X}
$$

e

$$
f\left(M_{n}\right) \underset{n \rightarrow \infty}{\longrightarrow} M_{Y} \neq p_{Y}
$$

Logo $f^{-1}\left(M_{Y}\right)=M_{X} \neq p_{X}$, e pela continuidade de $f^{-1}$ em $M_{Y}$, temos

$$
f^{-1}\left(f\left(M_{n}\right)\right)=M_{n} \underset{n \rightarrow \infty}{\longrightarrow} M_{X} \neq p_{X}
$$

o que é uma contradição.

Assim $f$ é contínua em $\mathcal{V}_{\omega}$ e de forma análoga o mesmo vale para $f^{-1}$. Daí $f$ é um homeomorfismo de $\mathcal{V}_{\omega}$ nele mesmo que leva órbitas de $X$ em órbitas de $Y$ e que coincide com $f_{D}$ no bordo $\partial \mathcal{V}_{\omega}$.

Vamos agora definir o homeomorfismo $f$ para uma vizinhança $\mathcal{V}_{\omega}$ onde $\omega=\gamma_{X}$ é uma órbita periódica atratora de $X$. Faremos a construção para uma região contendo uma órbita periódica finita, mas o procedimento para a construção do homeomorfismo para uma região contendo uma órbita periódica infinita é essencialmente o mesmo.

Seja $\gamma_{Y}$ a órbita periódica atratora de $Y$ contida em $\mathcal{V}_{\omega}$. Sejam $C_{1}$ e $C_{2}$ as curvas que formam o bordo de $\mathcal{V}_{\omega}$.

Considere os arcos da grade $[\Gamma, \Delta]$ que estão contidos no interior de $\mathcal{V}_{\omega}$ e são seções transversais para os campos $X$ e $Y$. Nomeie essas seções por $L_{0}, L_{1}, \ldots, L_{m}$.

Tome então pontos $E_{w}, w \in\{0,1, \ldots, m+1\}$, em $\gamma_{X}$, com $E_{m+1}=E_{0}=L_{0} \cap \gamma_{X} \mathrm{e}$ $E_{j}=L_{j} \cap \gamma_{X}, j=1,2, \ldots, m$.

De forma análoga teremos pontos $F_{u}, u \in\{0,1, \ldots, m+1\}$, em $C_{1}$ e $G_{v}, v \in$ $\{0,1, \ldots, m+1\}$, em $C_{2}$.

É fácil ver que da forma como as regiões canônicas são definidas e os homeomorfismos construídos, que dado $f_{D}$ o homeomorfismo definido em $D$, então esse homeomorfismo restrito as curvas $C_{1}$ e $C_{2}$ é tal que $f_{D}\left(F_{u}\right)=F_{u}$ e $f_{D}\left(G_{v}\right)=G_{v}$, para $u, v \in\{0,1, \ldots, m\}$.

Nós queremos definir um homeomorfismo $f$ que coincida com $f_{D}$ em $C_{1}$ e $C_{2}$ e que leve $\gamma_{X}$ em $\gamma_{Y}$. Vemos que com essas condições, $f$ deve levar o anel aberto limitado por $C_{1}$ e $\gamma_{X}$ no anel aberto limitado por $C_{1}$ e $\gamma_{Y}$, e deve levar o anel aberto limitado por $C_{2}$ e $\gamma_{X}$ no limitado por $C_{2}$ e $\gamma_{Y}$.

Defina então o homeomorfismo como $f(M)=f_{D}(M)$ se $M \in C_{1} \cup C_{2}$. Vamos definir $f$ no anel limitado por $C_{1}$ e $\gamma_{X}$.

Seja $\varphi(t, M, X)$ a trajetória de $X$ por $M \in C_{1}$. Conforme $t$ aumenta, a trajetória corta cada uma dessas seções transversais $L_{j}$ uma quantidade enumerável de vezes em 


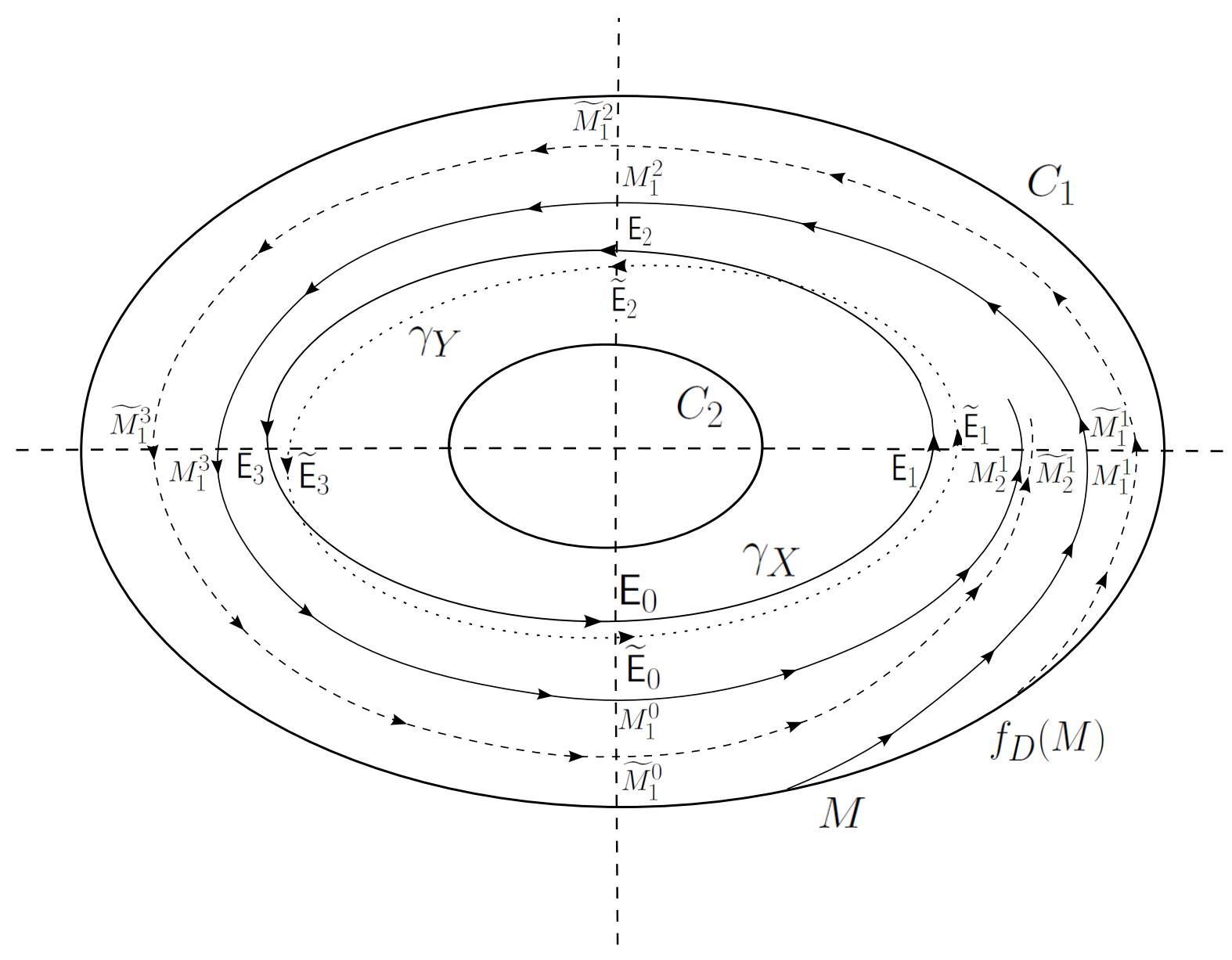

uma sequência monótona $\left(M_{n}^{j}\right)_{n \in \mathbb{N}}$, onde

$$
M_{n}^{j} \underset{n \rightarrow \infty}{\longrightarrow} E_{j}
$$

Assim a trajetória $\varphi(t, M, X)$ está particionada em um número infinito de arcos $\gamma_{n}^{j}=$ $M_{n}^{j} M_{n}^{j+1}, j=0,1, \ldots, m$ e $\gamma_{n}^{m+1}=M_{n}^{m+1} M_{n}^{0}$.

Faça o mesmo para $\varphi\left(t, f_{D}(M), Y\right)$ e sejam $\tilde{M}_{n}^{j}, \tilde{\gamma}_{n}^{j}$ e $\tilde{E}_{j}$.

Defina agora $f$ de forma que o arco fechado $\gamma_{n}^{j}$ seja levado em $\tilde{\gamma}_{n}^{j}$ pela razão comprimento de arco, isto é, se $M \in \gamma_{n}^{j}$, então $f(M)=\tilde{M}$ onde $\tilde{M} \in \tilde{\gamma}_{n}^{j}$ e

$$
\frac{\left|M M_{n}^{j+1}\right|}{\left|M_{n}^{j} M_{n}^{j+1}\right|}=\frac{\left|\tilde{M} \tilde{M}_{n}^{j+1}\right|}{\left|\tilde{M}_{n}^{j} \tilde{M}_{n}^{j+1}\right|} .
$$

Dessa forma definimos $f$ no disco limitado por $C_{1}$ e $\gamma_{X}$. Analogamente definimos $f$ para o disco limitado por $C_{2}$ e $\gamma_{X}$.

Para estender $f$ para a curva $\gamma_{X}$, parametrizamos $\gamma_{X}$ e $\gamma_{Y}$ por razão de comprimento de arco da seguinte maneira: para todo ponto $M \in E_{j} E_{j+1}$, definimos $f(M)=\tilde{M}$, onde $\tilde{M} \in \tilde{E}_{j} \tilde{E}_{j+1} \mathrm{com}$

$$
\frac{\left|E_{j} M\right|}{\left|E_{j} E_{j+1}\right|}=\frac{\left|\tilde{E}_{j} \tilde{M}\right|}{\left|\tilde{E}_{j} \tilde{E}_{j+1}\right|} .
$$


Precisamos agora mostrar que $f$ é um homeomorfismo em $\mathcal{V}_{\gamma_{X}}$. Claramente $f$ é sobrejetora. O fato de que $f$ e $f^{-1}$ são contínuas decorre do seguinte.

Cada ponto $M$ de $\mathcal{V}_{\gamma_{X}}$ pertence a algum arco de trajetória do campo $X$ em $\mathcal{V}_{\gamma_{X}}$. Sem perda de generalidade vamos $\operatorname{supor} M \in \gamma_{n_{0}}^{j_{0}}=M_{n_{0}}^{j_{0}} M_{n_{0}}^{j_{0}+1}$ e que existe uma sequência de pontos $\left(N_{n}\right)_{n \in \mathbb{N}}$ é tal que

$$
N_{n} \underset{n \rightarrow \infty}{\longrightarrow} M
$$

e $\left(M_{n}^{j_{0}} M_{n}^{j_{0}+1}\right)_{n \in \mathbb{N}}$ é a sequência de arcos correspondentes a $N_{n}$.

Por continuidade, é claro que

$$
\left|M_{n}^{j_{0}} M_{n}^{j_{0}+1}\right| \underset{n \rightarrow \infty}{\longrightarrow}\left|M_{n_{0}}^{j_{0}} M_{n_{0}}^{j_{0}+1}\right| .
$$

Logo, pelo lema 4.2 concluímos que $f\left(N_{n}\right) \rightarrow f(M)$ quando $n \rightarrow \infty$. O mesmo vale para $f^{-1}$.

Isso prova que $f$ é um homeomorfismo em $\mathcal{V}_{\gamma_{X}}$ que leva órbitas de $X$ em órbitas de $Y$ e que coincide com $f_{D}$ em $\partial \omega$. Como queríamos provar.

Fica então dessa forma definido um homeomorfismo para cada vizinhança de uma fonte ou de um poço em $\mathbb{D}$ que coincide com $f_{D}$ em seu pontos comuns..

Chame então de $h_{Y}$ o homeomorfismo de $\mathbb{D}$ nele mesmo obtido juntando os homeomorfismos $f_{D}$ com todos os homeomorfismos definidos nas vizinhanças das fontes e dos poços.

Portanto $h_{Y}$ é um homeomorfismo de $\mathbb{D}$ nele mesmo, que leva órbitas de $X$ em órbitas de $Y$, preserva a grade $[\Gamma, \Delta]$ e o bordo $\partial \mathbb{D}$.

Com a proposição acima provamos que todo $X \in \Sigma_{\Gamma, \Delta}$ é estruturalmente estável, o que concluí a demonstração do item (2) do teorema 3.1.

\subsection{Densidade}

As duas proposições apresentadas a seguir nos auxiliarão na demonstração do teorema 3.3 .

Proposição 4.3 Para todo $X \in \mathfrak{X}_{\Gamma, \Delta}$ a família a quatro parâmetros $X_{\alpha_{1}, \alpha_{2}, \nu, \beta}$ definida por

$$
X_{\alpha_{1}, \alpha_{2}, \nu, \beta}(x, y)=X(x, y)+\alpha_{1} \frac{\partial}{\partial x}+\alpha_{2} \frac{\partial}{\partial y}+\nu\left(x \frac{\partial}{\partial x}+y \frac{\partial}{\partial y}\right)+\beta\left(-y \frac{\partial}{\partial x}+x \frac{\partial}{\partial y}\right)
$$


intersecta o conjunto $\mathfrak{X}_{\Gamma, \Delta} \backslash \Sigma_{\Gamma, \Delta}\left(1 ; 1,2^{\prime}, 3\right) \cap \Sigma_{\Gamma, \Delta}(2 ; 2)$ em um conjunto de medida de Lebesgue nula em $\mathbb{R}^{4}$.

Demonstração: Vamos analisar o efeito dos parâmetros $\alpha_{1}, \alpha_{2}, \nu, \beta$ no retrato de fase de um campo $X \in \mathfrak{X}_{\Gamma, \Delta}$ alterando suas singularidades, tangências e órbita periódica no infinito.

Analisaremos primeiro as singularidades no infinito. Considere a expressão do campo de vetores $X_{\nu, \beta}$ nas coordenadas $(\theta, \rho)$

$$
\left(A_{1}^{\nu, \beta}(\theta, \rho)+\rho A_{0}^{\nu, \beta}(\theta)\right) \frac{\partial}{\partial \theta}-\rho\left(R_{1}^{\nu, \beta}(\theta, \rho)+\rho R_{0}^{\nu, \beta}(\theta)\right) \frac{\partial}{\partial \rho} .
$$

As singularidades no infinito de $X_{\beta, \nu}$ são os pontos da forma $(\theta, 0)$ onde

$$
A_{1}^{\nu, \beta}(\theta, 0)=0
$$

Suponha agora que $\left(\theta_{0}, 0\right)$ é uma singularidade no infinito para o campo com $\theta_{0} \neq \frac{\pi}{2} z$, $z \in \mathbb{Z}$. Calculando o jacobiano do campo no ponto encontramos

$$
\left(\begin{array}{cc}
\frac{\partial}{\partial \theta} A_{1}^{\nu, \beta}\left(\theta_{0}, 0\right) & \frac{\partial}{\partial \rho} A_{1}^{\nu, \beta}\left(\theta_{0}, 0\right)+A_{0}^{\nu, \beta}\left(\theta_{0}\right) \\
0 & -R_{1}^{\nu, \beta}\left(\theta_{0}, 0\right)
\end{array}\right) .
$$

Como a matriz acima é triangular, seus autovalores são os elementos da diagonal principal, portanto, para a singularidade no infinito $\left(\theta_{0}, 0\right)$ ser hiperbólica, devemos ter

$$
\left(\frac{\partial}{\partial \theta} A_{1}^{\nu, \beta}\left(\theta_{0}, 0\right)\right) R_{1}^{\nu, \beta}\left(\theta_{0}, 0\right) \neq 0
$$

As funções $A_{1}(\theta, 0)$ e $R_{1}(\theta, 0)$ de $X$ se relacionam com as funções $A_{1}^{\nu, \beta}(\theta, 0)$ e $R_{1}^{\nu, \beta}(\theta, 0)$ de $X_{\nu, \beta}$ da seguinte maneira

$$
\begin{aligned}
& A_{1}^{\nu, \beta}(\theta, 0)=\beta+A_{1}(\theta, 0), \\
& R_{1}^{\nu, \beta}(\theta, 0)=\nu+R_{1}(\theta, 0),
\end{aligned}
$$

o que nos garante a seguinte relação

$$
\begin{aligned}
& A_{1}^{\nu, \beta}(\theta, 0)=0 \Leftrightarrow \beta=-A_{1}(\theta, 0), \\
& R_{1}^{\nu, \beta}(\theta, 0)=0 \Leftrightarrow \nu=-R_{1}(\theta, 0) .
\end{aligned}
$$

Considere agora no espaço tridimensional $(\nu, \beta, \theta)$ a superfície formada pelas singula- 
ridades no infinito de $X_{\nu, \beta}$, ou seja

$$
S(\nu, \theta)=\left(\nu,-A_{1}(\theta, 0), \theta\right)
$$

Contida nessa superfície nós temos a curva

$$
C(\theta)=\left(-R_{1}(\theta, 0),-A_{1}(\theta, 0), \theta\right)
$$

formada pelas singularidades não hiperbólicas no infinito tais que $R_{1}^{\beta, \nu}(\theta, 0)$ se anula.

As funções $A_{1}(\theta, 0)$ e $R_{1}(\theta, 0)$ são contínuas e, restritas a cada intervalo da forma $\left[(k-1) \frac{\pi}{2}, k \frac{\pi}{2}\right], k \in\{1,2,3,4\}$, são quadráticas com suas expressões dadas por 2.19 e 2.20.

Portanto os pontos onde $\frac{\partial}{\partial \theta} A_{1}^{\beta, \nu}(\theta, 0)=0$ equivalem aos pontos críticos das funções $A_{1 k}(\theta)$ em cada intervalo $](k-1) \frac{\pi}{2}, k \frac{\pi}{2}[$.

Verifica-se facilmente que cada função $A_{1 k}(\theta)$ tem no máximo um ponto crítico em ]$(k-1) \frac{\pi}{2}, k \frac{\pi}{2}\left[\right.$. Logo a função $A_{1}(\theta, 0)$ tem no máximo quatro valores críticos no conjunto ] $0,2 \pi\left[\backslash\left\{\frac{\pi}{2}, \pi, 3 \frac{\pi}{2}\right\}\right.$.

Como queremos evitar também que as singularidades no infinito estejam localizadas na direção dos eixos $x$ e $y$ devemos desconsiderar os valores $\beta=A_{1}(\theta, 0)$ quando $\theta \in$ $\left\{0, \frac{\pi}{2}, \pi, 3 \frac{\pi}{2}\right\}$.

Assim os valores de $(\nu, \beta)$ para os quais temos uma singularidade não hiperbólica no infinito ou na direção dos eixos definem nove curvas na superfície $S$.

Projetando essas curvas no plano $(\nu, \beta)$ encontramos no máximo oito retas paralelas ao eixo $\nu$ e uma curva que é a projeção de $C$. Essa curva que é a projeção de $C$ é composta por quatro arcos de curvas analíticas, cada um correspondendo a um intervalo $\left[(k-1) \frac{\pi}{2}, k \frac{\pi}{2}\right], k \in\{1,2,3,4\}$. Chame de $c_{1}, c_{2}, \ldots, c_{9}$ essas retas e a curva que é projeção de $C$.

Observe agora que, para $X_{\alpha_{1}, \alpha_{2}, \nu, \beta}$ e $X_{\nu, \beta}$, vale

$$
\begin{aligned}
& A_{1}^{\alpha_{1}, \alpha_{2}, \nu, \beta}(\theta, 0)=A_{1}^{\nu, \beta}(\theta, 0), \\
& R_{1}^{\alpha_{1}, \alpha_{2}, \nu, \beta}(\theta, 0)=R_{1}^{\nu, \beta}(\theta, 0) .
\end{aligned}
$$

Logo, os pontos $\left(\alpha_{1}, \alpha_{2}, \nu, \beta\right)$ para os quais $X_{\alpha_{1}, \alpha_{2}, \nu, \beta} \notin \Sigma_{\Gamma, \Delta}(1,1)$ estão contidos na união finita de conjuntos de medida de Lebesgue nula em $\mathbb{R}^{4}$, cada um desses conjuntos sendo da forma $\left\{\left(\alpha_{1}, \alpha_{2}, \nu, \beta\right) \in \mathbb{R}^{4} \mid(\nu, \beta) \in c_{k}\right\}, k \in\{1,2, \ldots, 9\}$.

Vamos analisar agora a órbita no infinito. Um campo $X_{\nu, \beta}$ possui uma órbita periódica no infinito se $A_{1}^{\nu, \beta}(\theta, 0) \neq 0, \forall \theta \in[0,2 \pi]$. Sejam $M$ e $m$ o máximo e o mínimo respectivamente da função $-A_{1}(\theta, 0)$ restrita ao compacto $[0,2 \pi]$. Para todo $\beta \in \mathbb{R}, \beta \notin[m, M]$, o a função $A_{1}^{\nu, \beta}$ não se anula em $[0,2 \pi]$, $\operatorname{logo} X_{\nu, \beta}$ possui uma órbita periódica no infinito.

Para todo $(\nu, \beta) \in \mathbb{R}^{2}, \beta \notin[m, M]$, temos que a aplicação de retorno associada à órbita 
no infinito de $X_{\nu, \beta}$ é

$$
\pi_{\nu, \beta}^{\prime}(0)=\exp \left[\sum_{j=1}^{4} \int_{\theta_{j-1}}^{\theta_{j}}-\frac{\nu+R_{1 j}(\theta)}{\beta+A_{1 j}(\theta)} d \theta\right] .
$$

Pela proposição 2.9, concluímos que $\frac{\partial}{\partial \nu} \pi_{\nu, \beta}^{\prime}(0) \neq 0$. Fixado $\beta$ a função $\pi_{\nu, \beta}^{\prime}(0)$ é crescente ou decrescente quando variamos $\nu$, logo existe no máximo um valor de $\nu$ tal que a órbita periódica no infinito não é hiperbólica. Aplicando o teorema da função implícita para $\pi_{\nu, \beta}^{\prime}(0)=1$ encontramos que os pontos pontos $(\nu, \beta)$ onde $X_{\nu, \beta}$ tem uma órbita periódica não hiperbólica no infinito estão contidos em uma curva regular da forma $(\nu(\beta), \beta), \operatorname{com} \beta \notin[m, M]$.

Assim, da mesma forma como analisamos as singularidades no infinito, concluímos que os $\left(\alpha_{1}, \alpha_{2}, \nu, \beta\right)$ tal que $X_{\alpha_{1}, \alpha_{2}, \nu, \beta} \notin \Sigma_{\Gamma, \Delta}(2 ; 2)$ estão contidos no conjunto $\left\{\left(\alpha_{1}, \alpha_{2}, \nu, \beta\right) \in\right.$ $\left.\mathbb{R}^{4} \mid \nu=\nu(\beta)\right\}$, que é um conjunto de medida de Lebesgue nula em $\mathbb{R}^{4}$.

Para as tangências do sistema com a grade faremos a análise somente para uma tangência vertical, pois as tangências horizontais geram um resultado semelhante.

Fixe uma linha vertical $x=\gamma_{i}, i \in\{1,2, \ldots, n\}$. Para termos uma tangência vertical a primeira componente do campo de vetores $X_{\alpha_{1}, \alpha_{2}, \nu, \beta}$ deve se anular num ponto da forma $\left(\gamma_{i}, y\right)$. Logo

$$
\alpha_{1}+\nu \gamma_{i}-\beta y+P\left(\gamma_{i}, y\right)=0
$$

Assim, para cada $y_{0}$ fixado, os valores de $\left(\alpha_{1}, \alpha_{2}, \nu, \beta\right)$ para os quais $X_{\alpha_{1}, \alpha_{2}, \nu, \beta}$ tem uma tangência vertical em $\left(\gamma_{i}, y_{0}\right)$ estão contidos no hiperplano $H_{y_{0}}$ dado por

$$
\alpha_{1}+\nu \gamma_{i}-\beta y_{0}+P\left(\gamma_{i}, y_{0}\right)=0
$$

Portanto, para evitarmos as que as tangências ocorram nas esquinas da grade $[\Gamma, \Delta]$, devemos desconsiderar os $\left(\alpha_{1}, \alpha_{2}, \nu, \beta\right)$ contidos nos hiperplanos $H_{\delta_{j}}, j \in\{1,2, \ldots, m\}$.

Além disso, para termos sempre tangências parabólicas, considerando $\left(\alpha_{1}, \alpha_{2}, \nu, \beta\right) \in$ $H_{y}$, deve valer

$$
\frac{d}{d t}\left(\alpha_{1}+\nu \gamma_{i}-\beta y+P\left(\gamma_{i}, y\right)\right) \neq 0
$$

ou seja

$$
\left.P_{y}\left(\gamma_{i}, y\right)\right) \neq \beta
$$

Mas $P\left(\gamma_{k}, y\right)$ é uma função linear em cada segmento $\Delta_{j}, j \in\{1,2, \ldots, m\}$. Logo $P_{y}\left(\gamma_{i}, y\right)$ assume no máximo $m+1$ valores. Sejam $v_{1}, v_{2}, \ldots, v_{m+1}$ os valores que $P_{y}\left(\gamma_{i}, y\right)$ pode assumir. Assim, se desconsiderarmos os pontos nas reta $\left(\alpha_{1}, \alpha_{2}, \nu, v_{k}\right), k \in\{1,2, \ldots, m+$ $1\}$, garantimos que o campo $X_{\alpha_{1}, \alpha_{2}, \nu, \beta}$ possui somente tangências parabólicas com a reta da grade $x=\gamma_{i}$.

Fazendo esse processo para todas as retas verticais e horizontais, encontramos que os 
pontos $\left(\alpha_{1}, \alpha_{2}, \nu, \beta\right)$ tais que $X_{\alpha_{1}, \alpha_{2}, \nu, \beta} \notin \Sigma_{\Gamma, \Delta}(1 ; 3)$ estão contidos em um conjunto de medida nula em $\mathbb{R}^{4}$, pois ele é a união finita de retas e hiperplanos.

Para as singularidades finitas, considere uma célula $\Omega_{i j}$. Nela o campo de vetores é escrito como

$$
X(x, y)=A_{i j}\left(\begin{array}{l}
x \\
y
\end{array}\right)+b_{i j}
$$

Escreva

$$
A_{i j}=\left(\begin{array}{cc}
a_{11}^{i j} & a_{12}^{i j} \\
a_{21}^{i j} & a_{22}^{i j}
\end{array}\right), \quad b_{i j}=\left(\begin{array}{c}
b_{1}^{i j} \\
b_{2}^{i j}
\end{array}\right) .
$$

Dessa forma, na célula $\Omega_{i j}$, o campo de vetores $X_{\alpha_{1}, \alpha_{2}, \nu, \beta}$ é

$$
\left(\begin{array}{cc}
a_{11}^{i j}+\nu & a_{12}^{i j}-\beta \\
a_{21}^{i j}+\beta & a_{22}^{i j}+\nu
\end{array}\right)\left(\begin{array}{l}
x \\
y
\end{array}\right)+\left(\begin{array}{c}
b_{1}^{i j}+\alpha_{1} \\
b_{2}^{i j}+\alpha_{2}
\end{array}\right)
$$

Para termos singularidades não simples em $\Omega_{i j}$, devemos ter

$$
\operatorname{det}\left(\begin{array}{cc}
a_{11}^{i j}+\nu & a_{12}^{i j}-\beta \\
a_{21}^{i j}+\beta & a_{22}^{i j}+\nu
\end{array}\right)=0
$$

ou seja

$$
f_{i j}(\nu, \beta)=\nu^{2}+\beta^{2}+\operatorname{tr} A_{i j} \nu+\left(a_{21}^{i j}-a_{12}^{i j}\right) \beta+\operatorname{det} A_{i j}=0 .
$$

Essa é a equação de uma seção cônica, logo os valores $(\nu, \beta)$ que satisfazem a equação 4.2 podem estar contidos em uma elipse, uma parábola, uma hipérbole, retas, um ponto, ou ser o conjunto vazio. Em todos os casos, quando fixamos $(\nu, \beta)$, e tomamos a imagem de $\Omega_{i j}$ por $X_{\alpha_{1}, \alpha_{2}, \nu, \beta}$, encontramos um ponto, um segmento de reta ou uma semirreta. Logo, os valores de $\left(\alpha_{1}, \alpha_{2}, \nu, \beta\right)$ para os quais o campo de vetores $X_{\alpha_{1}, \alpha_{2}, \nu, \beta}$ possui uma singularidade não simples em $\Omega_{i j}$ está contido no conjunto $\left\{\left(\alpha_{1}, \alpha_{2}, \nu, \beta\right) \mid f_{i j}(\nu, \beta)=0\right\}$, que é um conjunto de medida de Lebesgue nula em $\mathbb{R}^{4}$.

Tome agora os pontos $(\nu, \beta)$ fora do conjunto $f_{i j}(\nu, \beta)=0$. Queremos evitar que apareçam singularidade em $\partial \Omega_{i j}$. Vamos fazer a análise supondo que estamos em uma célula finita, o outro caso é idêntico.

Tome $\left(\gamma_{i}, \delta_{j}\right)$ e $\left(\gamma_{i}, \delta_{j+1}\right)$ pontos de esquina da grade contidos em $\Omega_{i j}$. Para termos uma singularidade no segmento de reta da grade delimitado por esses pontos deve valer

$$
\left(\begin{array}{cc}
a_{11}^{i j}+\nu & a_{12}^{i j}-\beta \\
a_{21}^{i j}+\beta & a_{22}^{i j}+\nu
\end{array}\right)\left(\begin{array}{c}
\gamma_{i} \\
\delta_{j}+t\left(\delta_{j+1}-\delta_{j}\right)
\end{array}\right)+\left(\begin{array}{c}
b_{1}^{i j}+\alpha_{1} \\
b_{2}^{i j}+\alpha_{2}
\end{array}\right)=\left(\begin{array}{l}
0 \\
0
\end{array}\right)
$$

para $t \in[0,1]$. 
Se fizermos

$$
\begin{aligned}
& \alpha_{1}(\nu, \beta, t)=-\left(b_{1}^{i j}+\left(a_{11}^{i j}+\beta\right) \gamma_{i}+\left(a_{12}^{i j}+\nu\right)\left[\delta_{j}+t\left(\delta_{j+1}-\delta_{j}\right)\right]\right), \\
& \alpha_{2}(\nu, \beta, t)=-\left(b_{2}^{i j}+\left(a_{21}^{i j}+\beta\right) \gamma_{i}+\left(a_{22}^{i j}+\nu\right)\left[\delta_{j}+t\left(\delta_{j+1}-\delta_{j}\right)\right]\right),
\end{aligned}
$$

os $\left(\alpha_{1}, \alpha_{2}, \nu, \beta\right)$ tais que $X_{\alpha_{1}, \alpha_{2}, \nu, \beta}$ possui uma singularidade no segmento de reta da grade delimitado por $\left(\gamma_{i}, \delta_{j}\right)$ e $\left(\gamma_{i}, \delta_{j+1}\right)$ estão contidos na superfície regrada

$$
Z(\nu, \beta, t)=\left(\alpha_{1}(\nu, \beta, t), \alpha_{2}(\nu, \beta, t), \nu, \beta\right)
$$

que é um conjunto de medida nula em $\mathbb{R}^{4}$.

Repetindo o processo para os outros lados da célula e para as outras células, concluímos que os pontos $\left(\alpha_{1}, \alpha_{2}, \nu, \beta\right)$ tais que $X_{\alpha_{1}, \alpha_{2}, \nu, \beta} \notin \Sigma_{\Gamma, \Delta}\left(1 ; 2^{\prime}\right)$ estão contidos em um conjunto de medida de Lebesgue nula em $\mathbb{R}^{4}$.

Para terminarmos a demonstração do lema, basta tomarmos a união de todos conjuntos de medida nula encontrados.

Proposição 4.4 Para todo $X \in \Sigma_{\Gamma, \Delta}\left(1 ; 1,2^{\prime}, 3\right) \cap \Sigma_{\Gamma, \Delta}(2 ; 2)$ o campo vetorial rotacionado

$$
X_{\omega}(x, y)=R_{\omega} X(x, y)
$$

intersecta o conjunto $\mathfrak{X}_{\Gamma, \Delta}^{\prime}=\mathfrak{X}_{\Gamma, \Delta} \backslash \Sigma_{\Gamma, \Delta}$ em um conjunto de medida de Lebesgue nula em $\mathbb{R}$.

Demonstração: Vamos analisar como a rotação por $\omega$ age nos campos de vetores em questão.

Primeiro observe que as singularidades finitas não se alteram quando aplicamos uma rotação no campo de vetores $X$. Portanto os pontos singulares de $X$ e $X_{\omega}$ são os mesmos.

Além disso se escrevermos $X(x, y)=(P(x, y), Q(x, y))$, então para $\left(x_{0}, y_{0}\right)$ um ponto singular finito, temos

$$
D X\left(x_{0}, y_{0}\right)=\left(\begin{array}{ll}
P_{x}\left(x_{0}, y_{0}\right) & P_{y}\left(x_{0}, y_{0}\right) \\
Q_{x}\left(x_{0}, y_{0}\right) & Q_{y}\left(x_{0}, y_{0}\right)
\end{array}\right) .
$$

E para o campo $X_{\omega}(x, y)$, vale

$$
D X_{\omega}\left(x_{0}, y_{0}\right)=\left(\begin{array}{cc}
\cos \omega & \operatorname{sen} \omega \\
-\operatorname{sen} \omega & \cos \omega
\end{array}\right)\left(\begin{array}{ll}
P_{x}\left(x_{0}, y_{0}\right) & P_{y}\left(x_{0}, y_{0}\right) \\
Q_{x}\left(x_{0}, y_{0}\right) & Q_{y}\left(x_{0}, y_{0}\right)
\end{array}\right)
$$


Aplicando o determinante na igualdade acima, concluímos que

$$
\operatorname{det} D X\left(x_{0}, y_{0}\right)=\operatorname{det} D X_{\omega}\left(x_{0}, y_{0}\right)
$$

Logo depois de uma rotação por $\omega$, selas do campo $X$ continuam sendo selas e anti-selas continuam sendo anti-selas.

Se det $D X_{\omega}\left(x_{0}, y_{0}\right)<0$, então $\left(x_{0}, y_{0}\right)$ é uma singularidade hiperbólica.

Agora, se det $D X_{\omega}\left(x_{0}, y_{0}\right)>0$, devemos analisar $\operatorname{tr} D X_{\omega}\left(x_{0}, y_{0}\right)$ para podermos garantir a hiperbolicidade do ponto.

Observe que

$$
\operatorname{tr} D X_{\omega}\left(x_{0}, y_{0}\right)=\cos \omega\left(P_{x}\left(x_{0}, y_{0}\right)+Q_{y}\left(x_{0}, y_{0}\right)\right)+\operatorname{sen} \omega\left(P_{y}\left(x_{0}, y_{0}\right)-Q_{x}\left(x_{0}, y_{0}\right)\right)
$$

que pode ser reescrito como

$$
\operatorname{tr} D X_{\omega}\left(x_{0}, y_{0}\right)=\left\langle(\cos \omega, \operatorname{sen} \omega) ;\left(P_{x}\left(x_{0}, y_{0}\right)+Q_{y}\left(x_{0}, y_{0}\right), P_{y}\left(x_{0}, y_{0}\right)-Q_{x}\left(x_{0}, y_{0}\right)\right)\right\rangle
$$

Vamos mostrar que $\left(P_{x}\left(x_{0}, y_{0}\right)+Q_{y}\left(x_{0}, y_{0}\right), P_{y}\left(x_{0}, y_{0}\right)-Q_{x}\left(x_{0}, y_{0}\right)\right) \neq(0,0)$. Suponha o contrário, assim temos

$$
\begin{aligned}
& P_{x}\left(x_{0}, y_{0}\right)+Q_{y}\left(x_{0}, y_{0}\right)=0 \\
& P_{y}\left(x_{0}, y_{0}\right)-Q_{x}\left(x_{0}, y_{0}\right)=0 .
\end{aligned}
$$

Multiplicando a primeira linha por $P_{x}\left(x_{0}, y_{0}\right)$, a segunda linha por $P_{y}\left(x_{0}, y_{0}\right)$ e somando as duas encontramos

$$
\left(P_{x}\left(x_{0}, y_{0}\right)\right)^{2}+\left(P_{y}\left(x_{0}, y_{0}\right)\right)^{2}+P_{x}\left(x_{0}, y_{0}\right) Q_{y}\left(x_{0}, y_{0}\right)-P_{y}\left(x_{0}, y_{0}\right) Q_{x}\left(x_{0}, y_{0}\right)=0,
$$

ou

$$
\left(P_{x}\left(x_{0}, y_{0}\right)\right)^{2}+\left(P_{y}\left(x_{0}, y_{0}\right)\right)^{2}+\operatorname{det} D X_{\omega}\left(x_{0}, y_{0}\right)=0
$$

Como $\left(P_{x}\left(x_{0}, y_{0}\right)\right)^{2}+\left(P_{y}\left(x_{0}, y_{0}\right)\right)^{2} \geqslant 0$ e estamos supondo $\operatorname{det} D X_{\omega}\left(x_{0}, y_{0}\right)>0$, a igualdade 4.3 nunca é satisfeita, logo

$$
\left(P_{x}\left(x_{0}, y_{0}\right)+Q_{y}\left(x_{0}, y_{0}\right), P_{y}\left(x_{0}, y_{0}\right)-Q_{x}\left(x_{0}, y_{0}\right)\right) \neq(0,0)
$$

Daí, existem apenas dois valores de $\omega$ em $\left[0,2 \pi\left[\right.\right.$ para os quais $\operatorname{tr} D X_{\omega}\left(x_{0}, y_{0}\right)=0$.

Isso pode ser visto geometricamente como os dois ângulos $\omega_{1}$ e $\omega_{2}$ em $[0,2 \pi$ [ tais que 
$\left(\cos \omega_{i}, \operatorname{sen} \omega_{i}\right), i=1,2 ;$ são perpendiculares ao vetor

$$
\left(P_{x}\left(x_{0}, y_{0}\right)+Q_{y}\left(x_{0}, y_{0}\right), P_{y}\left(x_{0}, y_{0}\right)-Q_{x}\left(x_{0}, y_{0}\right)\right)
$$

Concluímos portanto que devemos remover apenas uma quantidade enumerável de valores de $\omega$, todos da forma $\omega_{i}+2 k \pi, i=1,2 ; k \in \mathbb{Z}$, para garantir que todas as singularidades finitas de $X_{\omega}$ são hiperbólicas.

Vamos agora estudar as tangências do campo com a grade $[\Gamma, \Delta]$. Analisaremos apenas as tangências com as linhas verticais da grade, a análise das tangências com as linhas horizontais é análoga.

Primeiro observe que se $X \in \mathfrak{X}_{\Gamma, \Delta}$, quando aplicamos a rotação no campo obtemos um novo campo $X_{\omega}=\left(P_{\omega}, Q_{\omega}\right)$, onde

$$
P_{\omega}(x, y)=\cos \omega P(x, y)+\operatorname{sen} \omega Q(x, y) .
$$

Logo dada uma linha vertical da grade $x=\gamma_{i}, i \in\{1,2, \ldots, n\}$, para para que ocorra uma tangência devemos ter $P_{\omega}\left(\gamma_{i}, y\right)=0$ para algum $y \in \mathbb{R}$. Ou seja

$$
\cos \omega P\left(\gamma_{i}, y\right)+\operatorname{sen} \omega Q\left(\gamma_{i}, y\right)=0
$$

Como $X \in \Sigma_{\Gamma, \Delta}\left(1 ; 1,2^{\prime}, 3\right)$, não existem pontos singulares na grade $[\Gamma, \Delta]$. Concluímos então que se $Q\left(\gamma_{i}, y\right)=0$, então $P\left(\gamma_{i}, y\right) \neq 0$ e

$$
P_{\omega}\left(\gamma_{i}, y\right)=0 \Leftrightarrow \omega=\frac{\pi}{2}+k \pi, \quad k \in \mathbb{Z}
$$

Considere agora $\omega \neq \frac{\pi}{2}+k \pi, k \in \mathbb{Z}$, e $Q\left(\gamma_{k}, y\right) \neq 0$. Assim, a equação anterior pode ser reescrita como

$$
P\left(\gamma_{i}, y\right)+\operatorname{tg} \omega Q\left(\gamma_{i}, y\right)=0
$$

ou ainda

$$
\operatorname{tg} \omega=-\frac{P\left(\gamma_{i}, y\right)}{Q\left(\gamma_{i}, y\right)}
$$

Observe que $Q\left(\gamma_{i}, y\right)$ é uma função linear por partes tendo como conjunto de descontinuidade de sua derivada os pontos $\left\{\delta_{1}, \delta_{2}, \ldots, \delta_{m}\right\}$. Como a função é linear em cada intervalo $\Delta_{j}, j=0,1, \ldots, m$, concluímos que em cada um desses intervalos a função $Q\left(\gamma_{j}, y\right)$ pode: ser nula em todo ponto, ter um único zero isolado ou nunca se anular. $\mathrm{O}$ conjunto $V=\left\{y \in \mathbb{R} \mid Q\left(\gamma_{k}, y\right)=0\right\}$ é então a união finita de intervalos fechados e pontos isolados, e portanto fechado.

Para todo $y \in U=\mathbb{R} \backslash V$ os valores

$$
\omega=\operatorname{arctg}\left(-\frac{P\left(\gamma_{i}, y\right)}{Q\left(\gamma_{i}, y\right)}\right)+k \pi, \quad k \in \mathbb{Z},
$$


são tais que $X_{\omega}$ possui uma tangência com a reta $x=\gamma_{i}$ em $\left(\gamma_{i}, y\right)$. Para evitarmos tangências com as esquinas da grade devemos remover os valores de $\omega\left(\delta_{k}\right), k \in\{1,2, \ldots, m\}$, que nesse caso é um conjunto enumerável de pontos.

Fazendo isso para todas as retas da grade $[\Gamma, \Delta]$, garantimos que a menos de um conjunto enumerável de pontos em $\mathbb{R}, X_{\omega} \in \Sigma_{\Gamma, \Delta}(1 ; 3)$.

No equador, as singularidades no infinito são dadas pela equação

$$
A_{\omega 1}(\theta, 0)=\cos \omega A_{1}(\theta, 0)-\operatorname{sen} \omega R_{1}(\theta, 0)=0 .
$$

Tome então a função

$$
f(\omega, \theta)=\cos \omega A_{1}(\theta, 0)-\operatorname{sen} \omega R_{1}(\theta, 0)
$$

se $f\left(\omega_{0}, \theta_{0}\right)=0$, então, pelo fato de $\left(A_{1}(\theta, 0), R_{1}(\theta, 0)\right) \neq(0,0)$ vale

$$
\frac{\partial}{\partial \omega} f(\omega, \theta)=-\operatorname{sen} \omega A_{1}(\theta, 0)-\cos \omega R_{1}(\theta, 0) \neq 0
$$

aplicando o teorema da função implícita em $f$, encontramos que existe uma função diferenciável $\omega(\theta)$ tal que $f(\omega(\theta), \theta)=0, \forall \theta \in[0,2 \pi]$.

Na verdade, temos uma quantidade enumerável de funções que satisfazem isso, dado que se fizermos $\omega_{k}(\theta)=\omega(\theta)+k \pi, k \in \mathbb{Z}$, então $f\left(\omega_{k}(\theta), \theta\right)=0, \forall \theta \in[0,2 \pi]$.

Diferenciando $f(\omega(\theta), \theta)$ com relação a $\theta$, encontramos que

$$
\left(\frac{\partial}{\partial \omega} f(\omega(\theta), \theta)\right) \omega^{\prime}(\theta)+\frac{\partial}{\partial \theta} f(\omega(\theta), \theta)=0
$$

e usando que $\frac{\partial}{\partial \omega} f(\omega(\theta), \theta) \neq 0$ e $\frac{\partial}{\partial \theta} f(\omega(\theta), \theta)=\frac{\partial}{\partial \theta} A_{1 \omega(\theta)}(\theta, 0)$ concluímos

$$
\frac{\partial}{\partial \theta} A_{1 \omega(\theta)}(\theta, 0)=0 \Leftrightarrow \omega^{\prime}(\theta)=0
$$

Assim as singularidades infinitas não hiperbólicas de $X_{\omega}$ aparecem nos pontos em que $\omega^{\prime}(\theta)=0$

Observe ainda que não teremos singulares infinitas de $X_{\omega}$ tais que $R_{1 \omega}(\theta, 0)=0$, pois isso forçaria o surgimento de um segmento de reta de singularidades em uma célula infinita limitada por um dos quadrantes.

Como $X \in \Sigma_{\Gamma, \Delta}\left(1 ; 1,2^{\prime}, 3\right)$, então para todo $\theta \in \mathbb{R}$ vale

$$
\left(A_{1}(\theta, 0), R_{1}(\theta, 0)\right) \neq(0,0)
$$


assim, se $R_{1}(\theta, 0)=0$, então

$$
A_{\omega 1}(\theta, 0)=\cos \omega A_{1}(\theta, 0)=0 \Leftrightarrow \omega=\frac{\pi}{2}+k \pi k \in \mathbb{Z} .
$$

Considere então $\omega \neq \frac{\pi}{2}+k \pi$ e $R_{1}(\theta, 0) \neq 0$, podemos então escrever

$$
A_{1}(\theta, 0)-\operatorname{tg} \omega R_{1}(\theta, 0)=0 \Leftrightarrow \operatorname{tg} \omega=\frac{A_{1}(\theta, 0)}{R_{1}(\theta, 0)} .
$$

Assim os valores de $\omega$ para os quais $X_{\omega}$ tem uma singularidade infinita em $(\theta, 0)$ são

$$
\omega_{k}(\theta)=\operatorname{arctg}\left(\frac{A_{1}(\theta, 0)}{R_{1}(\theta, 0)}\right)+k \pi k \in \mathbb{Z}
$$

Usando as expressões dadas em 2.19 e 2.20, e diferenciando a expressão anterior com relação a $\theta$, encontramos que, em cada quadrante, $\omega^{\prime}(\theta)=0$ no máximo em dois pontos.

Como não queremos, que as singularidades estejam nas direções dos eixos $x$ e $y$, devemos também remover os valores

$$
\omega(\theta)=\operatorname{arctg}\left(\frac{A_{1}(\theta, 0)}{R_{1}(\theta, 0)}\right)+k \pi,
$$

$\theta \in\left\{0, \frac{\pi}{2}, \pi, 3 \frac{\pi}{2}\right\}$

Concluímos portanto, que a menos de um conjunto enumerável de pontos $\omega, X_{\omega} \in$ $\Sigma_{\Gamma, \Delta}(1 ; 1)$.

Vamos analisar agora a órbita no infinito.

O infinito é uma órbita periódica se, e somente se, $A_{\omega 1}(\theta, 0) \neq 0, \forall \theta \in \mathbb{R}$. Pela expressão

$$
A_{\omega 1}(\theta, 0)=\cos \omega A_{1}(\theta, 0)-\operatorname{sen} \omega R_{1}(\theta, 0)
$$

é fácil observar que o conjunto

$$
W=\left\{\omega \in \mathbb{R} \mid A_{\omega 1}(\theta, 0) \neq 0 \quad \forall \theta \in \mathbb{R}\right\}
$$

ou é vazio ou é formado pela união enumerável de intervalos abertos disjuntos.

Se $W$ não for vazio, tome $I$ um dos intervalos que está contido no conjunto. Para todo $\omega \in I$ podemos então calcular a derivada da aplicação de retorno para a órbita periódica no infinito.

Pelas proposições 2.8 e 2.10, temos a seguinte expressão para a derivada da aplicação de retorno

$$
\pi_{\omega}^{\prime}(0)=\exp \left[\sum_{j=1}^{4} \int_{\theta_{j-1}}^{\theta_{j}}-\frac{R_{\omega 1 j}(\theta)}{A_{\omega 1 j}(\theta)} d \theta\right]
$$


que podemos escrever como

$$
\pi_{\omega}^{\prime}(0)=\exp \left[\sum_{j=1}^{4} \int_{\theta_{j-1}}^{\theta_{j}}-\frac{\operatorname{sen} \omega A_{1 j}(\theta)+\cos \omega R_{1 j}(\theta)}{\cos \omega A_{1 j}(\theta)-\operatorname{sen} \omega R_{1 j}(\theta)} d \theta\right] .
$$

Diferenciando essa expressão com relação a $\omega$ encontramos

$$
\frac{\partial}{\partial \omega} \pi_{\omega}^{\prime}(0)=\pi_{\omega}^{\prime}(0)\left(\sum_{j=1}^{4} \int_{\theta_{j-1}}^{\theta_{j}}-\frac{\left(A_{1 j}(\theta)\right)^{2}+\left(R_{1 j}(\theta)\right)^{2}}{\left(\cos \omega A_{1 j}(\theta)-\operatorname{sen} \omega R_{1 j}(\theta)\right)^{2}} d \theta\right)
$$

e dado que o campo $X \in \Sigma_{\Gamma, \Delta}\left(1 ; 1,2^{\prime}, 3\right)$, então para $i=1,2,3$, 4, vale

$$
\left(A_{1 j}(\theta)\right)^{2}+\left(R_{1 j}(\theta)\right)^{2} \neq 0
$$

donde concluímos que no intervalo $I$ a expressão $\frac{\partial}{\partial \omega} \pi_{\omega}^{\prime}(0)$ não se anula.

No intervalo $I$ a função $\pi_{\omega}^{\prime}(0)$ é portanto crescente ou decrescente, e temos no máximo um valor de $\omega$ onde a órbita periódica no infinito não é hiperbólica.

Como $W$ é formado pela união enumerável de intervalos abertos disjuntos, então devemos remover no máximo uma quantidade enumerável de valores de $\omega$ para garantir que a órbita periódica no infinito seja hiperbólica.

Considere agora as $[\Gamma, \Delta]$-conexões de separatrizes. Sejam $p_{S_{1}}$ e $p_{S_{2}}$ um par de pontos de sela de $X_{\omega_{0}}$ com uma conexão de selas $\varphi_{\omega_{0}}$, onde uma separatriz instável de $p_{S_{1}}$ e uma estável de $p_{S_{2}}$ coincidem. Existe então um intervalo aberto $I_{\omega_{0}}$, com $\omega_{0}$ no interior desse intervalo, tal que para todo $\omega \in I_{\omega_{0}}$, a separatriz instável de $p_{S_{1}}$ para $X_{\omega}$ não mais encontra a separatriz estável de $p_{S_{2}}$ se $\omega \neq \omega_{0}$. Logo os valores de $\omega$ para os quais existe uma conexão entre as selas $p_{S_{1}}$ e $p_{S_{2}}$ formam um conjunto discreto em $\mathbb{R}$, e portanto enumerável.

O mesmo processo pode ser feito quando consideramos $[\Gamma, \Delta]$-conexões de separatrizes que conectam pontos de esquina, pontos de tangência e pontos de sela. Como temos uma quantidade finita desses pontos, então os valores de $\omega$ para os quais $X_{\omega}$ possui uma $[\Gamma, \Delta]$-conexão de separatrizes estão contidos na união finita de conjuntos enumeráveis em $\mathbb{R}$.

Analisaremos agora as órbitas periódicas finitas. Sabemos, pela teoria de campos rodados [Duf53], que os pontos $p \in \mathbb{R}^{2}$ tais que $\varphi\left(t, p_{0}, X_{\omega}\right)$ é uma órbita periódica, formam regiões anulares no plano. Além disso, para cada ponto $p_{0}$ contido nessas regiões, existe um único $\omega_{0} \in\left[0, \pi\left[\right.\right.$ tal que $\varphi\left(t, p_{0}, X_{\omega_{0}}\right)$ é periódica.

Como o conjunto $\Gamma \times \Delta$ dos pontos de esquina da grade possui $n m$ elementos, então devemos retirar no máximo $n m$ valores de $\omega$ em $\left[0, \pi\left[\right.\right.$ para garantir que $X_{\omega}$ não possui órbitas periódicas passando por pontos de esquina. Logo, a menos de um conjunto enumerável de pontos $\omega$ em $\mathbb{R}, X_{\omega}$ possui todas as suas órbitas periódicas disjuntas de 

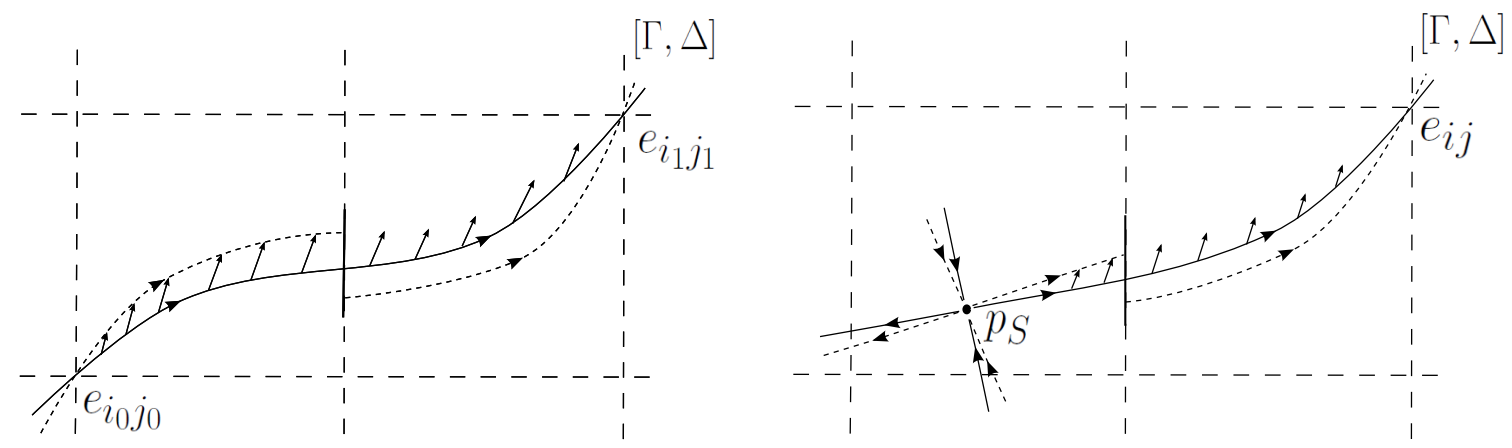

Figura 4.5: Desaparecimento de conexões de separatrizes através de uma rotação

$\Gamma \times \Delta$.

Pela análise que fizemos anteriormente para as $[\Gamma, \Delta]$-conexões de separatrizes, a menos de um conjunto enumerável de valores de $\omega$, garantimos que o campo rotacionado não possui conexões de tangência, e em particular, não possui órbitas periódicas tangenciando a grade $[\Gamma, \Delta]$.

Nos resta então analisar as órbitas periódicas que são transversais a grade $[\Gamma, \Delta]$ e que não possuem pontos de esquina.

Tome $p_{0}$ em $\mathbb{R}^{2}$ e $\omega_{0}$ em $\mathbb{R}$ tal que $\varphi\left(t, p_{0}, X_{\omega_{0}}\right)$ é uma órbita periódica que não possui pontos de esquina ou de tangência com a grade $[\Gamma, \Delta]$. Sem perda de generalidade, podemos supor que $p_{0}$ está contido em um pequeno segmento de reta $L_{0}$ em $[\Gamma, \Delta] \backslash \Gamma \times \Delta$ e $\omega_{0}=0$. O segmento $L_{0}$ é uma seção transversal para os campos $X_{\omega}$, para valores de $\omega$ pequenos.

Pela observação 2.3, podemos então definir uma função

$$
\Pi: L_{0} \times[-\epsilon, \epsilon] \rightarrow L_{0}
$$

tal que para todo $\omega \in[-\epsilon, \epsilon]$

$$
\Pi(p, \omega)
$$

é a aplicação de retorno do campo $X_{\omega}$ associada a seção transversal $L_{0}$. A função $\Pi$ é obtida compondo as aplicações de transição entre os lados das células, levando em consideração a órbita periódica $\varphi\left(t, p_{0}, X\right)$. De fato, se essa órbita corta a grade em pontos $p_{0}=E_{0}, E_{1}, \ldots, E_{k}$, tomando pequenos segmentos de reta $L_{j}$ em $[\Gamma, \Delta] \backslash \Gamma \times \Delta$, $E_{j} \in L_{j}$ e as aplicações de transição

$$
\pi_{j}: L_{j} \times[-\epsilon, \epsilon] \rightarrow L_{j+1}
$$

dadas pela observação 2.3 , então

$$
\Pi(p, \omega)=\pi_{k} \circ \pi_{k-1} \circ \ldots \circ \pi_{0}(p, \omega) .
$$




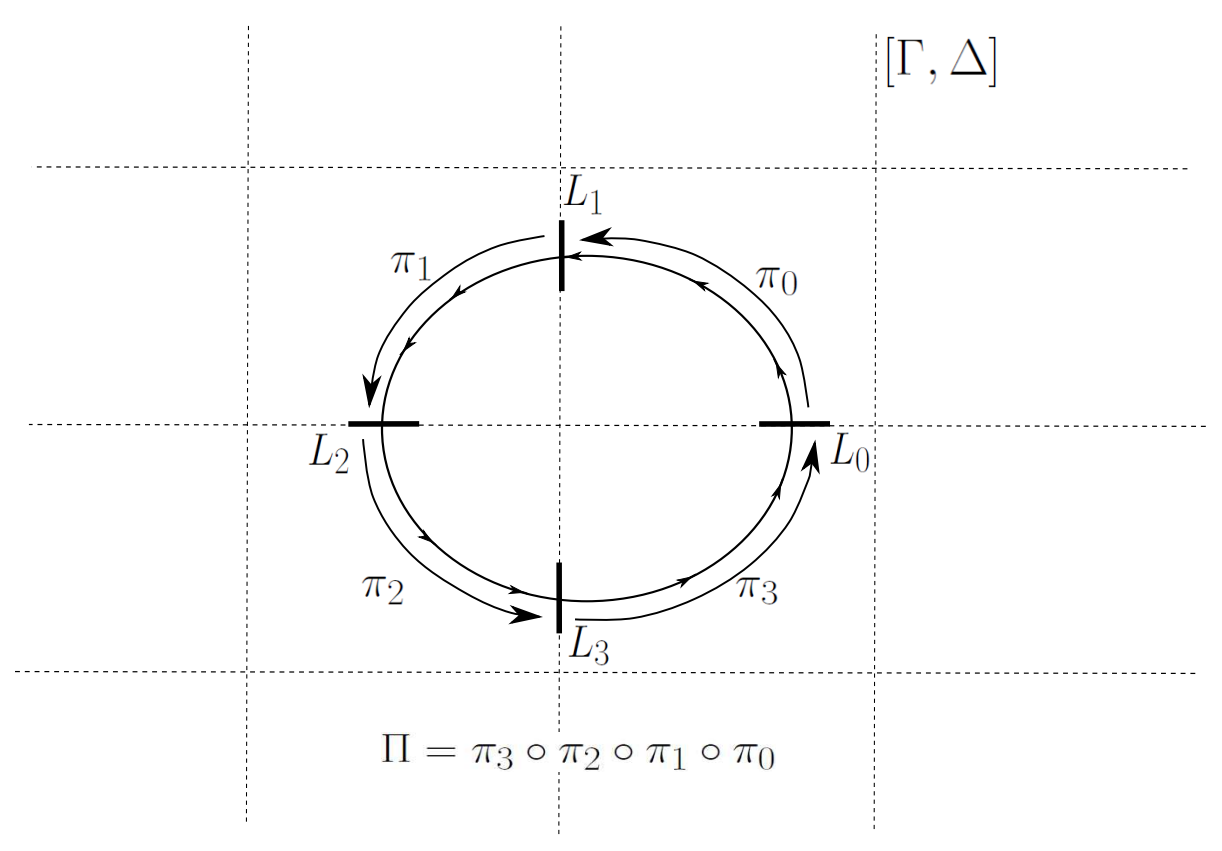

Figura 4.6: Aplicação П

Como cada uma dessas funções é analítica, então П é uma função analítica. Vamos mostrar agora que $\frac{\partial}{\partial \omega} \Pi(p, 0) \neq 0$.

Primeiro, vamos analisar a derivada da aplicação de transição $\pi_{j}$ com relação ao parâmetro $\omega$. Considere a órbita $\varphi\left(t, p_{0}, X\right)$, entrando na célula da grade transversalmente através do ponto $\varphi\left(t_{0}, p_{0}, X\right)=q_{0} \in L_{j}$ e saindo dessa célula transversalmente pelo ponto $\varphi\left(t_{1}, p_{0}, X\right)=q_{1} \in L_{j+1}$. Tome sobre a órbita $\varphi\left(t, p_{0}, X\right)$ a normal $\vec{n}(t), t \in\left[t_{0}, t_{1}\right]$, orientada positivamente com relação ao campo $X$.

Ao longo da normal $\vec{n}\left(t_{1}\right)$, tomamos coordenadas $s$, de modo que quando pensamos na aplicação de transição entre as seções transversais dadas pelos vetores $\vec{n}\left(t_{0}\right)$ e $\vec{n}\left(t_{1}\right)$, a imagem de $q_{0}$ por essa aplicação de transição é $q_{1}+s(\omega) \vec{n}\left(t_{1}\right)$. Logo, para chegarmos até a seção transversal $L_{1}$, ainda precisamos percorrer um tempo $\tau(s(\omega))$, onde essa função é dada pela definição 2.1 .

Assim, temos que a aplicação de transição $\pi_{j}\left(q_{0}, \omega\right)$ pode ser escrita como

$$
\varphi\left(\tau(s(\omega)), q_{1}+s(\omega) \vec{n}\left(t_{1}\right), X_{\omega}\right) .
$$

Derivando essa expressão com relação a $\omega$ nos dá

$$
\begin{aligned}
\frac{\partial}{\partial \omega}\left[\varphi\left(\tau(s(\omega)), q_{1}+s(\omega) \vec{n}\left(t_{1}\right), X_{\omega}\right)\right] & =\frac{\partial \varphi}{\partial t}\left(\tau(s(\omega)), q_{1}+s(\omega) \vec{n}\left(t_{1}\right), X_{\omega}\right) \frac{\partial \tau}{\partial s}(s(\omega)) \frac{\partial s}{\partial \omega}(\omega) \\
& \left.+\frac{\partial \varphi}{\partial p}\left(\tau(s(\omega)), q_{1}+s(\omega) \vec{n}\left(t_{1}\right), X_{\omega}\right)\right) \frac{\partial s}{\partial \omega}(\omega) \vec{n}\left(t_{1}\right) \\
& +\frac{\partial \varphi}{\partial \omega}\left(\tau(s(\omega)), q_{1}+s(\omega) \vec{n}\left(t_{1}\right), X_{\omega}\right) .
\end{aligned}
$$




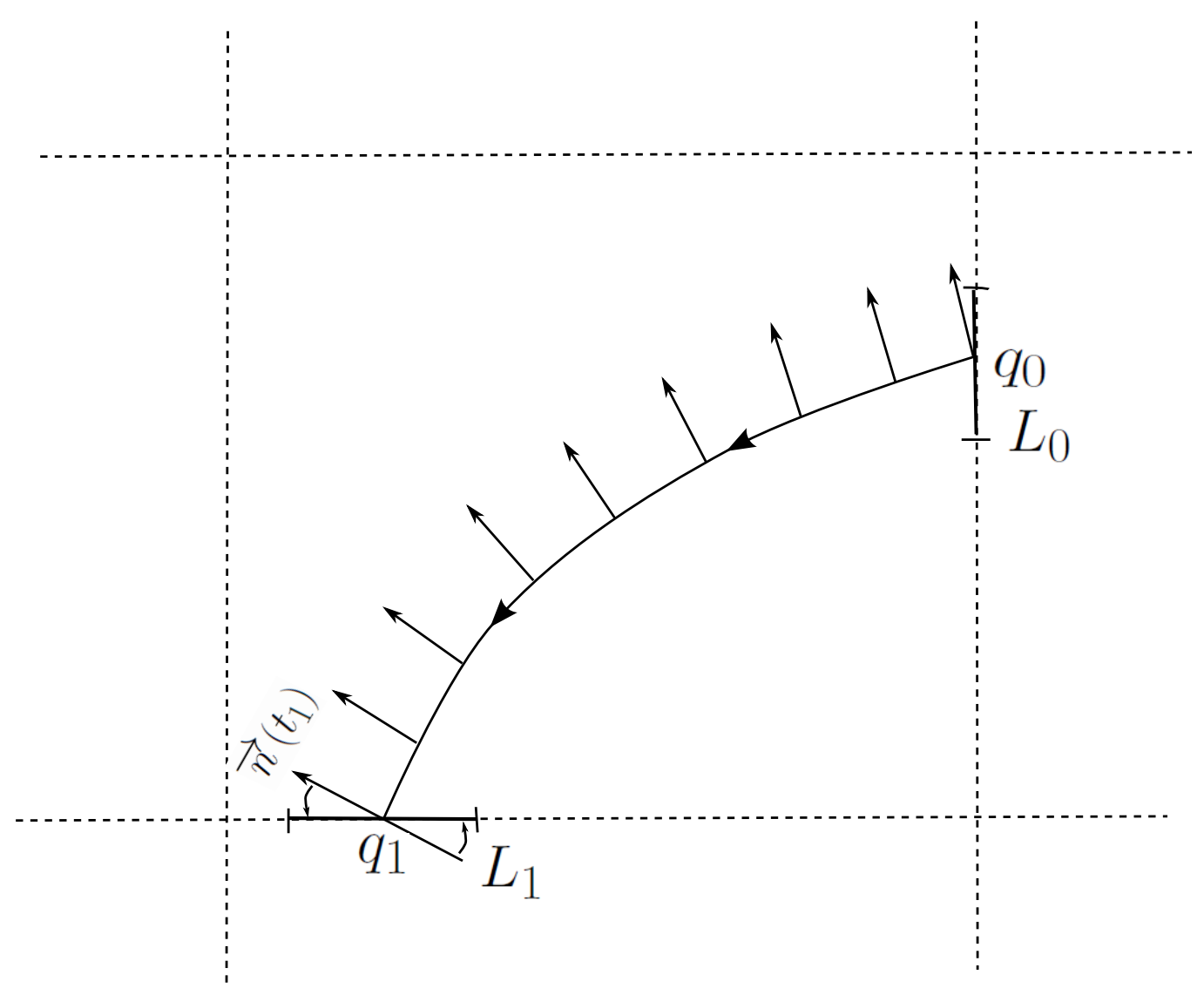

Figura 4.7: Aplicaçao $\pi_{j}$

Calculando para $\omega=0$, temos

$$
\frac{\partial}{\partial \omega}\left[\varphi\left(\tau(s(0)), q_{1}+s(0) \vec{n}\left(t_{1}\right), X\right)\right]=\left[X\left(q_{1}\right) \frac{\partial \tau}{\partial s}(s(\omega))+\vec{n}\left(t_{1}\right)\right] \frac{\partial s}{\partial \omega}(\omega),
$$

onde usamos que $\left.\frac{\partial \varphi}{\partial p}\left(0, q_{1}, X\right)\right)=I d$ e $\frac{\partial \varphi}{\partial \omega}\left(0, q_{1}, X\right)=0$.

Agora observe que $X\left(q_{1}\right) \frac{\partial \tau}{\partial s}(s(\omega))+\vec{n}\left(t_{1}\right)$ é um vetor não nulo e colinear a seção $L_{1}$. Observe também que, calculando a derivada com relação a $\omega$ da função $s(\omega)$, considerando a família a um parâmetro $X_{\omega}$, a proposição 2.4 nos dá $\frac{\partial s}{\partial \omega}(\omega)>0$. Disso, concluímos que nessas condições, $\frac{\partial \pi_{j}}{\partial \omega}\left(q_{0}, 0\right)>0$.

Se considerarmos

$$
T_{j-1}(p, \omega)=\pi_{j-1} \circ \ldots \circ \pi_{0}\left(p_{0}, \omega\right),
$$

então para a composição $\pi_{j}\left(T_{j-1}(p, \omega), \omega\right)$ vale

$$
\frac{\partial}{\partial \omega}\left[\pi_{j}\left(T_{j-1}\left(p_{0}, \omega\right), \omega\right)\right]=\pi_{j}^{\prime}\left(T_{j-1}\left(p_{0}, \omega\right), \omega\right) \frac{\partial}{\partial \omega}\left[T_{j-1}\left(p_{0}, \omega\right)\right]+\frac{\partial \pi_{1}}{\partial \omega}\left(T_{j-1}\left(p_{0}, \omega\right), \omega\right),
$$

onde $\pi_{j}^{\prime}$ é a derivada da aplicação de transição dada pela proposição 2.3.

Como $\pi_{j}^{\prime}>0$ sempre, então se $\frac{\partial}{\partial \omega}\left[T_{j-1}\left(p_{0}, \omega\right)\right]>0$ e $\frac{\partial \pi_{1}}{\partial \omega}\left(T_{j-1}\left(p_{0}, \omega\right), \omega\right)>0$, podemos 
concluir que

$$
\frac{\partial}{\partial \omega}\left[\pi_{j}\left(T_{j-1}\left(p_{0}, \omega\right), \omega\right)\right]>0, \quad \forall j \in\{0,1, \ldots, k\}
$$

e, em particular

$$
\frac{\partial}{\partial \omega} \Pi(p, 0)>0
$$

Mas isso é facilmente verificado aplicando o princípio da indução finita às funções $\pi_{j}\left(T_{j-1}(p, \omega), \omega\right)$, levando em consideração que $\pi_{j}^{\prime}>0$ e $\frac{\partial \pi_{j}}{\partial \omega}\left(q_{0}, 0\right)>0$.

Assim, se tomarmos $\sigma_{0}: I \rightarrow L_{0}$ uma parametrização da seção transversal $L_{0}$ com $\sigma_{0}(0)=p_{0}$, usando as coordenadas locais $s$, e aplicando o teorema da função implícita em

$$
\Pi(s, \omega)-s
$$

como $\Pi(0,0)=0$ e $\frac{\partial \Pi}{\partial \omega}(0,0) \neq 0$, temos que existe uma função analítica $\omega(s)$, definida em uma vizinhança $J$ de 0 , tal que

$$
\Pi(s, \omega(s))-s=0
$$

para todo $s \in J$. Além disso, se derivarmos a expressão anterior com relação a $s$, vale

$$
\frac{\partial \Pi}{\partial s}(s, \omega(s))+\frac{\partial \Pi}{\partial \omega}(s, \omega(s)) \omega^{\prime}(s)=1,
$$

e como $\frac{\partial \Pi}{\partial \omega}(s, \omega(s)) \neq 0$, concluímos

$$
\frac{\partial \Pi}{\partial s}(s, \omega(s))=1 \Leftrightarrow \omega^{\prime}(s)=0
$$

Ou seja, os valores de $\omega$ para os quais $\varphi\left(t, \gamma_{0}(s), X_{\omega(s)}\right)$ é uma órbita periódica não hiperbólica, correspondem aos valores critícos da função $\omega(s)$. Como essa função é analítica, concluímos que a menos de um conjunto enumerável de valores de $\omega, X_{\omega}$ não possui órbitas periódicas finitas não hiperbólicas.

Finalizamos a demonstração tomando a união finita de todos os conjuntos encontrados anteriormente. Logo, a menos de um conjunto enumerável de valores de $\omega$, temos $X_{\omega} \notin$ $\mathfrak{X}_{\Gamma, \Delta}^{\prime}$

Com as duas proposições anteriores podemos demonstrar o teorema 3.3.

Teorema 3.3 Para todo $X \in \mathfrak{X}_{\Gamma, \Delta}$ a família a cinco parâmetros definida por

$$
X_{\alpha_{1}, \alpha_{2}, \nu, \beta, \omega}(x, y)=R_{\omega}\left[X(x, y)+\alpha_{1} \frac{\partial}{\partial x}+\alpha_{2} \frac{\partial}{\partial y}+\nu\left(x \frac{\partial}{\partial x}+y \frac{\partial}{\partial y}\right)+\beta\left(-y \frac{\partial}{\partial x}+x \frac{\partial}{\partial y}\right)\right]
$$


intersecta o conjunto fechado $\mathfrak{X}_{\Gamma, \Delta}^{\prime}=\mathfrak{X}_{\Gamma, \Delta} \backslash \Sigma_{\Gamma, \Delta}$ em um conjunto de medida de Lebesgue nula.

Demonstração: Tome $X \in \mathfrak{X}_{\Gamma, \Delta}$ e considere o conjunto

$$
\mathcal{U}(X)=\left\{\left(\alpha_{1}, \alpha_{1}, \nu, \beta, \omega\right) \in \mathbb{R}^{5} \mid R_{\omega}\left(X_{\alpha_{1}, \alpha_{2}, \nu, \beta}\right) \in \mathfrak{X}_{\Gamma, \Delta}^{\prime}\right\}
$$

Precisamos mostrar que $m_{5}(\mathcal{U}(X))=0$, onde $m_{5}$ representa a medida de Lebesgue em $\mathbb{R}^{5}$. Observe que o conjunto $\mathcal{U}(X)$ é fechado e portanto Lebesgue mensurável. De fato, se tomarmos a função contínua

$$
\begin{aligned}
F: \mathbb{R}^{5} & \longrightarrow \mathbb{R}^{N(\Gamma, \Delta)} \\
\left(\alpha_{1}, \alpha_{2}, \nu, \beta, \omega\right) & \longmapsto R_{\omega}\left(X_{\alpha_{1}, \alpha_{2}, \nu, \beta}\right),
\end{aligned}
$$

então $\mathcal{U}(X)=F^{-1}\left(\mathfrak{X}_{\Gamma, \Delta}^{\prime}\right)$, onde $\mathfrak{X}_{\Gamma, \Delta}^{\prime}$ é um conjunto fechado. Assim, temos

$$
m_{5}(\mathcal{U}(X))=\int_{\mathbb{R}^{5}} \mathcal{X}(\mathcal{U}(X)) d m_{5}
$$

onde $\mathcal{X}(A)$ denota a função característica do conjunto $A$. Aplicando o teorema de Fubini, [Rud87], para a função $\mathcal{X}(\mathcal{U}(X))$, temos

$$
m_{5}(\mathcal{U}(X))=\int_{\mathbb{R}^{4}}\left(\int_{\mathbb{R}} \mathcal{X}\left(\mathcal{U}\left(X ; \alpha_{1}, \alpha_{2}, \nu, \beta\right)\right) d m_{1}\right) d m_{4}
$$

onde $m_{1}$ e $m_{4}$ são respectivamente a medida de Lebesgue em $\mathbb{R}$ e $\mathbb{R}^{4}$, e

$$
\mathcal{U}\left(X ; \alpha_{1}, \alpha_{2}, \nu, \beta\right)=\left\{\omega \in \mathbb{R} \mid R_{\omega}\left(X_{\alpha_{1}, \alpha_{2}, \nu, \beta}\right) \in \mathfrak{X}_{\Gamma, \Delta}^{\prime}\right\}
$$

Se tomarmos

$$
\mathcal{V}(X)=\left\{\left(\alpha_{1}, \alpha_{1}, \nu, \beta\right) \in \mathbb{R}^{4} \mid X_{\alpha_{1}, \alpha_{2}, \nu, \beta} \in \mathfrak{X}_{\Gamma, \Delta} \backslash \Sigma_{\Gamma, \Delta}\left(1 ; 1,2^{\prime}, 3\right) \cap \Sigma_{\Gamma, \Delta}(2 ; 2)\right\}
$$

então temos que $m_{5}(\mathcal{U}(X))$ é igual a

$$
\int_{\mathbb{R}^{4} \backslash \mathcal{V}(X)}\left(\int_{\mathbb{R}} \mathcal{X}\left(\mathcal{U}\left(X ; \alpha_{1}, \alpha_{2}, \nu, \beta\right)\right) d m_{1}\right) d m_{4}+\int_{\mathcal{V}(X)}\left(\int_{\mathbb{R}} \mathcal{X}\left(\mathcal{U}\left(X ; \alpha_{1}, \alpha_{2}, \nu, \beta\right)\right) d m_{1}\right) d m_{4}
$$

Pela proposição 4.3, a segunda integral se anula, e pela proposição 4.4, a primeira integral se anula. Concluímos portanto que $m_{5}(\mathcal{U}(X))=0$, como queríamos. 
Teorema 3.2 O conjunto aberto $\Sigma_{\Gamma, \Delta}=\Sigma_{\Gamma, \Delta}(1) \cap \Sigma_{\Gamma, \Delta}(2) \cap \Sigma_{\Gamma, \Delta}(3)$ possui medida de Lebesgue total, em particular $\Sigma_{\Gamma, \Delta}$ é denso em $\mathfrak{X}_{\Gamma, \Delta}$.

Demonstração: Vamos mostrar que $m_{N}\left(\mathfrak{X}_{\Gamma, \Delta}^{\prime}\right)=0$, onde $m_{N}$ representa a medida de Lebesgue em $\mathbb{R}^{N(\Gamma, \Delta)}$. Considere a função contínua

$$
\begin{aligned}
F: \mathbb{R}^{N(\Gamma, \Delta)} \times \mathbb{R}^{5} \longrightarrow \mathbb{R}^{N(\Gamma, \Delta)} \\
\left(X,\left(\alpha_{1}, \alpha_{2}, \nu, \beta, \omega\right)\right) \longmapsto R_{\omega}\left(X_{\alpha_{1}, \alpha_{2}, \nu, \beta}\right),
\end{aligned}
$$

e defina a função

$$
G\left(X,\left(\alpha_{1}, \alpha_{2}, \nu, \beta, \omega\right)\right)=\mathcal{X}\left(\mathfrak{X}_{\Gamma, \Delta}^{\prime}\right) \circ F\left(X,\left(\alpha_{1}, \alpha_{2}, \nu, \beta, \omega\right)\right) .
$$

Usando o teorema 3.2 , temos

$$
\int_{\mathbb{R}^{N(\Gamma, \Delta)}}\left(\int_{\mathbb{R}^{5}} G\left(X,\left(\alpha_{1}, \alpha_{2}, \nu, \beta, \omega\right)\right) d m_{5}\right) d m_{N}=0 .
$$

Assim, aplicando o teorema de Fubini para a função $G$, concluímos que

$$
\int_{\mathbb{R}^{5}}\left(\int_{\mathbb{R}^{N(\Gamma, \Delta)}} G\left(X,\left(\alpha_{1}, \alpha_{2}, \nu, \beta, \omega\right)\right) d m_{N}\right) d m_{5}=0 .
$$

Logo, a menos de um conjunto de medida de Lebesgue nula em $\mathbb{R}^{5}$, temos

$$
\int_{\mathbb{R}^{N(\Gamma, \Delta)}} G\left(X,\left(\alpha_{1}, \alpha_{2}, \nu, \beta, \omega\right)\right) d m_{N}=0
$$

Fixe $\left(\alpha_{1}, \alpha_{2}, \nu, \beta, \omega\right) \in \mathbb{R}^{5}$ tal que a expressão anterior seja válida. Agora observe que

$$
G\left(X,\left(\alpha_{1}, \alpha_{2}, \nu, \beta, \omega\right)\right)=1 \Leftrightarrow R_{\omega}\left(X_{\alpha_{1}, \alpha_{2}, \nu, \beta}\right)=Y
$$

$\operatorname{com} Y \in \mathfrak{X}_{\Gamma, \Delta}^{\prime}$. Ou equivalentemente

$$
X(x, y)=R_{(-\omega)}(Y)(x, y)-\alpha_{1} \frac{\partial}{\partial x}-\alpha_{2} \frac{\partial}{\partial y}-\nu\left(x \frac{\partial}{\partial x}+y \frac{\partial}{\partial y}\right)-\beta\left(-y \frac{\partial}{\partial x}+x \frac{\partial}{\partial y}\right)
$$

Seja $X_{0}$ o campo de vetores em $\mathfrak{X}_{\Gamma, \Delta}$ com a seguinte expressão

$$
X_{0}(x, y)=-\alpha_{1} \frac{\partial}{\partial x}-\alpha_{2} \frac{\partial}{\partial y}-\nu\left(x \frac{\partial}{\partial x}+y \frac{\partial}{\partial y}\right)-\beta\left(-y \frac{\partial}{\partial x}+x \frac{\partial}{\partial y}\right) .
$$


Então, com essa notação, 4.5 é escrita

$$
X=R_{(-\omega)}(Y)+X_{0}
$$

Disso concluímos que, para o ponto $\left(\alpha_{1}, \alpha_{2}, \nu, \beta, \omega\right)$ fixado, $G\left(X,\left(\alpha_{1}, \alpha_{2}, \nu, \beta, \omega\right)\right)$ é a função característica do conjunto $R_{(-\omega)}\left(\mathfrak{X}_{\Gamma, \Delta}^{\prime}\right)+X_{0}$, onde estamos considerando $R_{(-\omega)}$ uma transformação linear em $\mathbb{R}^{N(\Gamma, \Delta)}$.

Como a medida de Lebesgue é invariante por translações e vale 4.4, concluímos

$$
0=m_{N}\left(R_{(-\omega)}\left(\mathfrak{X}_{\Gamma, \Delta}^{\prime}\right)+X_{0}\right)=\left|\operatorname{det} R_{(-\omega)}\right| m_{N}\left(\mathfrak{X}_{\Gamma, \Delta}^{\prime}\right)
$$

e como $\left|\operatorname{det} R_{(-\omega)}\right| \neq 0$ vale

$$
m_{N}\left(\mathfrak{X}_{\Gamma, \Delta}^{\prime}\right)=0
$$

Isso prova que o conjunto $\Sigma_{\Gamma, \Delta}$ possui medida de Lebesgue total e em particular é denso em $\mathfrak{X}_{\Gamma, \Delta}$. De fato, se $\Sigma_{\Gamma, \Delta}$ não fosse denso, existiriam $X \in \mathfrak{X}_{\Gamma, \Delta}$ e $\delta>0$ tal que $B_{\delta}(X) \subseteq \mathfrak{X}_{\Gamma, \Delta}^{\prime}$. Mas isso geraria uma contradição com o fato de $\mathfrak{X}_{\Gamma, \Delta}^{\prime}$ ter medida nula. Como queríamos. 


\section{Capítulo 5}

\section{Conclusões}

Neste trabalho estudamos a classe $\mathfrak{X}_{\Gamma, \Delta}$ de campos de vetores seccionalmente lineares no plano. Fixados os conjuntos $\Gamma=\left\{\gamma_{1}<\gamma_{2}<\ldots<\gamma_{n}\right\}$ e $\Delta=\left\{\delta_{1}<\delta_{2}<\ldots<\delta_{m}\right\}$, $\mathfrak{X}_{\Gamma, \Delta}$ é um espaço vetorial de dimensão finita, cujos elementos são campos de vetores cujas componentes possuem as seguintes expressões

$$
\begin{aligned}
& P(x, y)=a_{1}+b_{11} x+b_{12} y+\sum_{i=1}^{n} c_{1}^{i}\left|x-\gamma_{i}\right|+\sum_{j=1}^{m} d_{1}^{j}\left|y-\delta_{j}\right|, \\
& Q(x, y)=a_{2}+b_{21} x+b_{22} y+\sum_{i=1}^{n} c_{2}^{i}\left|x-\gamma_{i}\right|+\sum_{j=1}^{m} d_{2}^{j}\left|y-\delta_{j}\right| .
\end{aligned}
$$

Através do método da compactificação de Poincaré, ver proposição 2.6, construção apropriada para lidar com a não compacidade do plano, demos uma definição precisa de estabilidade estrutural para campos em $\mathfrak{X}_{\Gamma, \Delta}$, ver definição 3.1.

Baseados no artigo de Jorge Sotomayor e Ronaldo Garcia [SG03], e inspirados pelas condições encontradas no teorema de Andronov-Pontryagin-Peixoto, apresentamos, na seção 3.2, condições suficientes para que um campo de vetores seja estruturalmente estável em $\mathfrak{X}_{\Gamma, \Delta}$, impondo condições sobre as órbitas periódicas, as singularidades e as separatrizes dos sistemas 5.1, finitas e infinitas.

Os campos que satisfazem essas condições definem um conjunto $\Sigma_{\Gamma, \Delta}$. Demonstramos, ver teorema 3.1, que esse conjunto é aberto e denso em $\mathfrak{X}_{\Gamma, \Delta}$.

Para a demonstração da abertura, usamos o método das regiões canônicas para campos suaves encontrado no artigo [PP59] e o adaptamos para a classe de campos seccionalmente lineares 5.1. Vimos que apenas três dos cinco tipos de regiões canônicas encontradas por Maurício Peixoto aparecem nos campos em $\Sigma_{\Gamma, \Delta}$.

No teorema 4.2, construímos detalhadamente os homeomorfismos para todos os tipos de regiões canônicas, observando que devemos defini-los em uma sequência adequada, 
para enfim obtermos os homeomorfismos que estabelecem a equivalência topológica entre campos de vetores próximos a $X$ em $\Sigma_{\Gamma, \Delta}$.

Através do estudo da família a cinco parâmetros

$$
X_{\alpha_{1}, \alpha_{2}, \nu, \beta, \omega}(x, y)=R_{\omega}\left[X(x, y)+\alpha_{1} \frac{\partial}{\partial x}+\alpha_{2} \frac{\partial}{\partial y}+\nu\left(x \frac{\partial}{\partial x}+y \frac{\partial}{\partial y}\right)+\beta\left(-y \frac{\partial}{\partial x}+x \frac{\partial}{\partial y}\right)\right]
$$

pelo teorema 3.3, usando os métodos encontrados em [Sot81], provamos que o conjunto $\Sigma_{\Gamma, \Delta}$ é denso em $\mathfrak{X}_{\Gamma, \Delta}$. Para tanto, construímos algumas funções dependendo dos parâmetros $\alpha_{1}, \alpha_{2}, \nu, \beta, \omega$ e estimamos a medida do conjunto de seus valores críticos. Devido ao fato dessas funções serem seccionalmente analíticas, provamos que tais conjuntos possuem medida nula sem recorrer ao Teorema de Sard.

Neste trabalho, devido a estrutura de $\mathfrak{X}_{\Gamma, \Delta}$, fomos mais adiante e provamos que $\Sigma_{\Gamma, \Delta}$ é um conjunto de medida de Lebesgue total.

Alguns aspectos abordados no artigo [SG03] não foram analisados nesse trabalho, como por exemplo, a demonstração de que o conjunto $\Sigma_{\Gamma, \Delta}$ é igual ao conjunto $\mathscr{S}^{r}$ dos campos ditos regularmente estruturalmente estáveis, ficando ainda em aberto demonstrar que $\mathscr{S}_{\Gamma, \Delta}=\Sigma_{\Gamma, \Delta}$.

Acreditamos que este trabalho seja uma referência útil e um convite para estudos futuros sobre as bifurcações de sistemas seccionalmente lineares no plano. Assunto que é de interesse tanto matemático teórico quanto aplicado, e já possui uma vasta literatura, como por exemplo os trabalhos [Gom00, Ter00, FPMT98, FPMT02, BM13, Pan02, LP12, LP99]. 


\section{Referências Bibliográficas}

[ALGM73] A. Andronov, E. Leontovich, I. Gordon, e G. Maier, Theory of bifurcations of dynamical systems on a plane, Israel Program for Scientific Translations, John Wiley, 1973.

[BM13] D. C. Braga e L. F. Mello, Limit cycles in a family of discontinuous piecewise linear differential systems with two zones in the plane, Nonlinear Dynamics 73 (2013), 1283-1288.

[CL55] E. A. Coddington e N. Levinson, Theory of ordinary differential equations, McGraw-Hill, 1955.

[CL91] L. Chua e R. Lum, Generic properties of continuous piecewise-linear vector field in $\mathbb{R}^{2}$, IEEE Trans. Circuits Systems 38 (1991), no. 9, 1043-1066.

[Duf53] G. F. D. Duff, Limit-cycles and rotated vector fields, Annals of Mathematics (1953), 15-31.

[FPMT98] E. Freire, E. Ponce, F. R. Muñoz, e F. Torres, Bifurcation sets of continuous piecewise linear systems with two zones, International Journal of Bifurcation and Chaos 8 (1998), no. 11, 2073-2097.

[FPMT02] _ _ Bifurcation sets of symmetrical continuous piecewise linear systems with three zones, International Journal of Bifurcation and Chaos 12 (2002), no. $8,1675-1702$.

[Gom00] A. J. Gomes, Seleção contínua de campos de vetores, Dissertação de mestrado, Universidade Federal de Goiás, 2000.

[LP99] J. Llibre e E. Ponce., Bifurcation of a periodic orbit from infinity in planar piecewise linear vector fields, Nonlinear Anal. 36 (1999), no. 5, 623-653, Ser. B: Real World Appl.

[LP12]__ Three nested limit cycles in discontinuous piecewise linear differential systems with two zones, Dynam. Contin. Discrete Impuls. Systems (2012), no. $19,325-335$, Ser. B. 
[Pan02] D. Panazzolo, Tame semiflows for piecewise linear vector fields, Ann. de L'Institut Fourier 52 (2002), no. 6, 1593-1628.

[Pei62] M. M. Peixoto, Structural stability on two-dimensional manifolds, Topology 1 (1962), 101-120.

[PP59] M. M. Peixoto e M. C. Peixoto, Structural stability in the plane with enlarged boundary conditions, Anais da Academia Brasileira de Ciências 31 (1959), no. 9, 135-160.

[Rud87] W. Rudin, Real and complex analysis, 3 ed., McGraw Hill, 1987.

[SG03] J. Sotomayor e R. Garcia, Structural stability of piecewise-linear vector fields, Journal of Differential Equations 192 (2003), 553-565.

[Sot79] J. Sotomayor, Lições de equações diferenciais ordinárias, Projeto Euclides, Instituto de Matemática Pura e Aplicada, 1979.

[Sot81] _ Curvas definidas por equações diferenciais no plano, Colóquio Brasileiro de Matemática, Instituto de Matemática Pura e Aplicada, 1981.

[Sot85]_ Stable planar polynomial vector fields, Rev. Mat. Iberoamericana 1 $(1985)$.

[Ter00] A. E. Teruel, Clasificación topológica de uma familia de campos vectoriales lineales a trozos simétricos en el plano, Tese de doutorado, Universitat Autònoma de Barcelona, 2000. 


\section{Índice Remissivo}

$$
\begin{aligned}
& {[\Gamma, \Delta], 6} \\
& \Gamma \times \Delta, 6 \\
& \Sigma_{\Gamma, \Delta}, 39 \\
& \Sigma_{\Gamma, \Delta}(1), 38 \\
& \quad \Sigma_{\Gamma, \Delta}(1 ; 1), 38 \\
& \quad \Sigma_{\Gamma, \Delta}\left(1 ; 1,2^{\prime}, 3\right), 38 \\
& \quad \Sigma_{\Gamma, \Delta}(1 ; 2), 38 \\
& \quad \Sigma_{\Gamma, \Delta}(1 ; 3), 38
\end{aligned}
$$$$
\Sigma_{\Gamma, \Delta}(2), 38
$$$$
\Sigma_{\Gamma, \Delta}(2 ; 1), 38
$$$$
\Sigma_{\Gamma, \Delta}(2 ; 2), 38
$$

$\Sigma_{\Gamma, \Delta}(3), 39$

$\mathbb{D}, 22$

$\mathbb{H}_{+}, 18$

$\mathbb{H}_{-}, 18$

$\mathbb{S}^{1}, 18$

$\mathbb{S}^{2}, 18$

$\mathcal{N}, 18$

$\mathfrak{X}_{\Gamma, \Delta}, 6$

$\mathscr{S}_{\Gamma, \Delta}, 37$

$\pi, 11$

$\tau, 10$

$\tilde{\tau}, 11$

órbita periódica

finita, 22

infinita, 22

aplicação de transição, 11

célula $\Omega_{i j}, 6$

compactificação Poincaré

na esfera, 21

no disco, 22

conexões de separatrizes, 38 conjunto de bifurcação, 40

continuidade do fluxo

com relação aos campos, 9

com relação aos parâmetros, 8

com relação as condições iniciais, 8

estabilidade estrutural, 37

grade compactificada, 22

pontos de esquina, 6

regiões canônicas, 58

tipos de, 59

rotação

campo de vetores, 34

separatrizes, 58

singularidade

anti-sela, 22

finita, 22

hiperbólica, 22

infinita, 22

sela, 22

simples, 22 